2019-09-03

\title{
Memory Influences Visual Cognition across Multiple Functional States of Interactive Cortical Dynamics
}

Schendan, $\mathrm{H}$

http://hdl.handle.net/10026.1/14733

10.1016/bs.plm.2019.07.007

Psychology of Learning and Motivation: Advances in Research and Theory

Elsevier

All content in PEARL is protected by copyright law. Author manuscripts are made available in accordance with publisher policies. Please cite only the published version using the details provided on the item record or document. In the absence of an open licence (e.g. Creative Commons), permissions for further reuse of content should be sought from the publisher or author. 
Running head: Cortical Dynamics of Human Object Memory

\section{ACCEPTED MANUSCRIPT}

\section{Psychology of Learning and Motivation \\ DOI: 10.1016/bs.plm.2019.07.007}

(accepted 29/06/2019; in press, 2019, v. 71)

Memory Influences Visual Cognition across Multiple Functional States of Interactive Cortical Dynamics

Haline E. Schendan

University of Plymouth

Corresponding Author: Haline E. Schendan

School of Psychology, University of Plymouth

Drake Circus, Plymouth, Devon, UK PL4 8AA

Tel: 01752 584804, Fax: 01752584800

Email: haline.schendan@gmail.com 


\begin{abstract}
Memory supports a wide range of abilities from categorical perception to goal-directed behavior, such as decision-making and episodic recognition. Memory activates fast and surprisingly accurately and even when information is ambiguous or impoverished (i.e., showing object constancy). This paper proposes the multiple-state interactive (MUSI) account of object cognition that attempts to explain how sensory stimulation activates memory across multiple functional states of neural dynamics, including automatic and strategic mental simulation mechanisms that can ground cognition in modal information processing. A key novel hypothesis of this account is 'multiple-function regional activity': The same neuronal population can contribute to multiple brain states, depending upon the dominant set of inputs at that time. In state 1, the initial fast bottom-up pass through posterior neocortex happens between $95 \mathrm{~ms}$ and $250 \mathrm{~ms}$, with knowledge supporting categorical perception by $120 \mathrm{~ms}$. In state 2, starting around 150 to $230 \mathrm{~ms}$, a sustained state of iterative activation of object-sensitive cortex involves bottom-up, recurrent, and feedback interactions with frontoparietal cortex. This supports higher cognitive functions (e.g., those associated with decision-making) even under ambiguous or impoverished conditions, phenomenological consciousness, and automatic mental simulation. In the latest state so far identified, state $\mathrm{M}$, starting around 300 to $500 \mathrm{~ms}$, large-scale cortical network interactions, including between multiple networks (e.g., control, salience, and especially default mode), further modulate posterior cortex. This supports elaborated cognition based on earlier processing, including episodic memory, strategic mental simulation, decision evaluation, creativity, and access consciousness. Convergent evidence is reviewed from cognitive neuroscience of object cognition, decision-making, memory, and mental imagery that support this account and define the brain regions and time course of these neural dynamics.
\end{abstract}

\title{
Key words:
}

visual perception; decision making; long-term memory; object cognition; event-related potential 
Human memory is activated rapidly, within $1 \mathrm{~s}$, to support a wide range of abilities from categorical perception to goal-directed behavior, such as decision-making and episodic recognition. Memory activation is not only fast but also surprisingly accurate and robust even when stimulus information is ambiguous or impoverished (i.e., showing object constancy, e.g., categorizing a dog regardless of viewing conditions). This paper proposes the multiple-state interactive (MUSI) account of visual object cognition that attempts to explain how sensory stimulation activates memory across multiple functional states of neural dynamics, including mental simulation mechanisms that ground cognition in modal information processing. These states build upon each other over time, thereby successively enabling increasingly more sophisticated cognitive abilities. The central idea of this account is that posterior cortical areas are activated at different times in stimulus processing during multiple "states" of cortical neural dynamics that perform distinct functions (Schendan \& Ganis, 2012; Schendan \& Kutas, 2007a; Schendan \& Maher, 2009; Schendan \& Stern, 2008). Certain neural processes characterize each state, distinguish between states, and determine the types of global functions for cognition that can happen in each. Key differences between states are in interactive cortical dynamics that can occur between the posterior areas and feedback, recurrent, reentrant and recursive computations, especially those involving frontoparietal regions. So far, three states have been proposed. Each state is associated with a major hypothesis about the types of cortical dynamics and their function in cognition. The MUSI account is based on convergent cognitive neuroscience studies of visual object perception, cognition and memory but can also explain the dynamics of cognition in general, including for other sensory modalities, though details would differ.

\section{Overview of MUSI account}

\subsection{Brain state: Definition and relevance}

A brain 'state' has been defined as synchronous neural firing reflected in the electrical activity of the brain (Brown, 2006). The MUSI account expands this to define a brain 'state' as large-scale global, neurophysiological activity that differs qualitatively in structure and function from other brain states. The particular brain structures involved in a state are part of each state's definition. MUSI focuses on synchronous brain states revealed using event-related brain potentials (ERPs). However, whether this activity is synchronous or not may vary with the state and its function, as variations in spike rate instead of synchrony of V1 neurons is associated with perceptual organization, awareness and attention (Lamme, 2003; Lamme, Super, Landman, Roelfsema, \& Spekreijse, 2000).

The idea of distinct brain states of neuronal activation helps advance cognitive neuroscience for several reasons. 1) Despite decades of discussion of brain states, theoretical accounts of them are scant (Brown, 2006, 2012). 2) States capture important observations from dynamic, large-scale neurophysiological responses that have not yet been articulated within theoretical neuroscience. 3 ) States are important for relating brain activity to the mind. The MUSI account describes processing times and functional properties that distinguish between some brain states, so methods with sufficient temporal resolution (e.g., ERPs) are necessary to study them; note, methods with poor temporal resolution (e.g., functional magnetic resonance imaging, fMRI) may reflect multiple temporally overlapping states, leading to erroneous interpretations of function. For example, different states are associated with different patterns of neural responses, which, if smeared across a time period, would yield a mixed and potentially misleading pattern. 4) Bayesian, adaptive resonance theory (ART), and other computational theories posit feedback processes are critical for cognition (Fazl, Grossberg, \& Mingolla, 2009; Friston, 2010), and evidence suggests that different states of feedback have different functions (Lamme \& Roelfsema, 2000). 


\subsection{Multiple-function regional activity: An overarching hypothesis}

In its current form, the MUSI account describes three states for visual object perception and cognition, and 'multiple-function regional activity' is a key novel hypothesis: The same regional neuronal population can contribute to multiple brain states, depending upon the dominant set of inputs to the region at that time. Specifically, the 3 essential brain states hypothesized so far to support healthy human cognition include activity within a neuronal population (e.g., cortical area such as the fusiform face area, FFA) that contributes different functions in different states. These functions are determined by the inputs that dominate the neurophysiological response of the cortical region (e.g., FFA), such as bottom-up, recurrent, and feedback or top-down (David, Harrison, \& Friston, 2005). The key point is that the neuronal population (or cortical area) is activated at different times in stimulus processing via different neuronal processes. Further, this multi-faceted activation pattern thereby results in different neural computations and thus different functional outputs and associated roles in cognition for different states. The MUSI account has focused on the structural level of a cerebral cortical area, which is a 2-3 mmm thick region of gray matter composed of multiple neuronal layers (six for neocortex) that performs a function that is distinct from other such areas (e.g., area V4 is necessary for color perception, whereas area V5 is necessary for motion perception), and cortical areas are organized hierarchically from simpler to more complex processing in the feedforward processing direction with multiple, intertwined processing streams and extensive interareal and feedback processes (Felleman \& Van Essen, 1991) The neurophysiological state can theoretically be defined from the cortical area down to the neuronal level, a more holistic goal for future work. Already, similar accounts have been proposed at the neuronal level in primary visual area V1, as in classical versus non-classical receptive fields of the same neuron (Lamme, 2003; Lamme \& Roelfsema, 2000).

Neurally-realistic computational modelling of event-related electromagnetic potentials (David et al., 2005; David et al., 2006) supports the MUSI account and suggests the dynamics of a neurophysiological state from neuronal to cortical area levels of brain architecture. Specifically, a neural mass model of hierarchically arranged cortical areas has been developed with feedforward, feedback, and lateral connections (David et al., 2005). In line with state 1 in the MUSI account, when feedforward inputs are sufficiently strong, excitatory interneuron responses saturate, resulting in an inherently stable state. The evoked responses generated by the model resemble early evoked potentials, such as the face-specific N170 (David et al., 2006). In contrast, and in line with MUSI state 2, when feedback or lateral (or recurrent) connections are added to the model, cortical dynamics change. Neurophysiological potentials increase in duration and show damped oscillations when top-down inputs are relatively smaller, and responses resemble later endogenous potentials, such as the N400 or P300, indicating that re-entrant dynamics within cortical hierarchies underlie the late potentials. In contrast, when top-down inputs increase above a threshold, a phase-transition occurs and time-locked oscillatory dynamics dominate and stabilize and become maximal around $500 \mathrm{~ms}$. Such late stable oscillatory dynamics may underlie state M in the MUSI account. Bilateral connections additionally cause zero-lag phase-locking among cortical areas. This is consistent with the MUSI idea that a state can involve multiple cortical areas, such as prefrontal and inferotemporal cortices.

In addition, when empirical data are used to estimate the parameters in this computational model and dynamic causal modeling of specific cortical areas is used, an increase in feedforward connectivity along the ventral visual pathway is associated with neurophysiological potentials resembling the face-specific N170 (David et al., 2006), consistent with MUSI state 1. Moreover, using a similar model with auditory oddball paradigm data, standards (e.g., frequent high tones) 
and oddballs (e.g., rare low tones) differ for the first time when feedback processes begin to contribute during later neurophysiological potentials (i.e., state 2), specifically the mismatch negativity, but not during earlier potentials (i.e., state 1), such as the N100 (David et al., 2006); note, addition of lateral (recurrent) connections was not superior to feedback connections alone, indicating feedback connections are most important for the functional processes underlying later neurophysiological potentials. Further, learning-related changes in feedforward and feedbackward connections drive the development of later potentials (David et al., 2006), indicating that learning is related to feedback processing during the second state of cortical dynamics. This is consistent with the MUSI account of state 2 .

\subsection{MUSI states 1, 2 and M: Overview and main hypotheses}

MUSI also aligns with the hierarchy of convergent and divergent cortical anatomy and convergence/ divergence zones (A. R. Damasio, 1989; Meyer \& Damasio, 2009), which indicates that states typically occur in a particular sequential order (David et al., 2005). Processing in a previous state needs to start before processes in the next state that depend upon that state can start. States can overlap in time. In fact, source estimation suggests state 1 can continue throughout later states, presumably because the processes continue to be needed for later states (Schendan \& Lucia, 2010).

The MUSI account was developed primarily to explain the cortical dynamics supporting tasks that emphasize ventral visual pathway processing, knowledge and memory, such as category decisions (e.g., 'what is the object?') and recognition (e.g., 'have I seen that specific object before?'). In line with the hypothesis of multiple-function regional activity, the MUSI account hypothesizes that states 1 and 2 both involve activation in object-sensitive occipitotemporal cortex (Schendan \& Kutas, 2007a; Schendan \& Stern, 2008).

\subsubsection{State 1}

The main state 1 hypothesis is the following. In this state, the fast bottom-up pass through this occipitotemporal cortex happens between 95 to $115 \mathrm{~ms}$ to around 150 to $250 \mathrm{~ms}$ (Fig. 1). In state 1, categorical perception happens along the ventral visual pathway, and this predominantly feedforward information processing in state 1 can be sufficient under unambiguous, optimal conditions to achieve higher cognition (e.g., a decision) in state 2.

\subsubsection{State 2}

The main state 2 hypothesis is the following. A second state of object knowledge activity takes place between $\sim 150-230 \mathrm{~ms}$ and lasting until around $500 \mathrm{~ms}$ or later (Figure 2), with timing varying with visual input and task demands. At this time, a sustained state of iterative activation of object-sensitive occipitotemporal cortex involves bottom-up, recurrent, and feedback interactions among these areas and with other brain structures, especially prefrontal and occipitoparietal cortex (David et al., 2005; Fazl et al., 2009; Schendan \& Kutas, 2002, 2007a; Schendan \& Maher, 2009). In state 2, higher cognition primarily occurs for the first time; that is, cognitive functions associated with actual decision-making (including under ambiguous or impoverished conditions), consciousness (especially phenomenological consciousness), and automatic mental simulation that grounds cognition, memory and meaning in modal information processing.

\subsubsection{State $M$}

While initial version of the MUSI account focused on states 1 and 2 (Schendan \& Kutas, 2007a; Schendan \& Maher, 2009), a third state, M (for multiple network), has been proposed recently (Schendan \& Ganis, 2012; 2015). The main hypothesis is the following. In state M, a default mode network accomplishes internal evaluation of decision and memory processes, and 
this regulates large-scale cortical network interactions among multiple networks (e.g., frontoparietal control, salience) and can further modulate posterior cortex. In state M, large-scale anti-correlated network activity (e.g., control, salience, and default mode) and their interactions support even higher-order cognitive functions or 'elaborated cognition' based on earlier, especially state 2, processing. Default mode network contributions include episodic memory retrieval and encoding, strategic mental imagery or simulation, decision evaluation, divergent thinking for creativity, and access consciousness. State $\mathrm{M}$ processing has been considered to be post-decision or post-categorization, with the decision or categorization instead happening earlier in state 2 (Folstein \& Van Petten, 2008; J. S. Johnson \& Olshausen, 2003, 2005; Ritter, Simson, Vaughan, $\&$ Friedman, 1979). State $M$ elaborates on state 2 processes and can influence and change the ultimate final decision, category, or motor behavior, as well as support episodic memory retrieval and encoding and strategic cognitive abilities, such as mental rotation, and other processes of creativity, such as divergent thinking using a default mode network and multiple large-scale network interactions (Vartanian et al., 2018). Accomplishing this can include a third activation of modal processing cortex (state $\mathrm{M}$ multi ple-function regional activity) where information is processed, stored and encoded, such as in occipitotemporal cortex.

\subsubsection{State 2 and $M$ Networks}

The frontoparietal regions that predominate in state 2 include those in executive control and dorsal attention networks, which are active task networks that are anti-correlated with the default mode network regions that support the internal evaluation, memory, access consciousness and creativity functions in state M (Spreng, Sepulcre, Turner, Stevens, \& Schacter, 2013), further supporting functional dissociations between states 2 and M (Ganis, Schendan, \& Kosslyn, 2007; Schendan \& Ganis, 2015; Schendan \& Kutas, 2003; Schendan \& Lucia, 2010; Schendan \& Stern, 2007, 2008). However, while the default mode network dominates for the functions so far identified for state $\mathrm{M}$, this state is labeled $\mathrm{M}$ because it involves multiple network interactions, and this multiplicity suggests future work will further subdivide state $\mathrm{M}$ into multiple states. For example, a slow wave may be better characterized as a late response planning and working memory state (Schendan \& Ganis, 2015).

\subsection{Consciousness}

Debates about the neural architecture of consciousness are independent of this account, but the MUSI account favors the view that recurrent processes and feedback, especially from frontoparietal to posterior cortex, are key to consciousness (Lamme, 2003). In this vein, MUSI proposes that state 2 predominantly supports phenomenological (a.k.a. phenomenal) consciousness, which is brief, susceptible and not readily reportable and associated with iconic sensory memory, and state $\mathrm{M}$ predominantly supports access (or report) consciousness, which is readily reportable and associated with working memory. That feedback is key to phenomenological consciousness would make MUSI a higher-order theory of consciousness, and the ideas and evidence for MUSI are consistent with other challenges to first-order theories $(\mathrm{H}$. C. Lau \& Passingham, 2007; Ledoux \& Brown, 2017). Nonetheless, within the domain of emotion, for which subcortical structures are necessary and have a core role, first-order theories are increasingly compelling and influential in mental health (Alcaro, Carta, \& Panksepp, 2017; Panksepp \& Biven, 2012) and in comprehensive theories of the brain basis of object cognition, memory, meaning, consciousness, self and embodied cognition (A. R. Damasio, 2010). Whether of cortical or subcortical origin, (a) feedback, reentrant, recursive and recurrent neuronal pathways and (b) synchronous ensembles of such neurons that are bound together for information processing (e.g., object features) have been proposed to be necessary for consciousness (A. R. Damasio, 
2010). MUSI thus takes a hybrid view that core or primary modal processing (e.g., state 1) can contribute to some degree of consciousness, but requires certain types of recurrent and feedback processes in the neural architecture and certain environmental circumstances (e.g., stimuli, task) in order to become conscious. MUSI also proposes that different states (e.g., state 1 vs. 2) contribute in different ways to consciousness, as constrained by their recurrent and feedback architectures; the quality (or qualia) of consciousness varies by state. For example, in state 1, feedforward activity triggers automatic recurrent and feedback processes along the ventral visual pathway, and such automatic feedback may support a more rudimentary form of consciousness than is possible in state 2 when feedback also includes frontoparietal contributions (e.g., 'elementary' vs. 'standard' phenomenological consciousness in states 1 vs. 2, respectively). In such a hybrid version, MUSI hypothesizes that state 2 has a greater role in phenomenological consciousness and can influence access consciousness in state $M$ more than state 1 . In short, the quality and qualia of consciousness transform progressively into more advanced and complex forms as processing proceeds from state to state.

\subsection{Object Constancy}

People can categorize (e.g., a dog) and recognize objects as familiar (e.g., the dog I saw in the park yesterday) fast and accurately. However, their performance can become slower and/or less accurate as sensory information about an object differs more and more from previous experience or the learning situation. Nonetheless, accuracy continues to be good despite remarkably wide variations in sensory input from the original learning experience. For example, people categorize and recognize objects accurately despite unusual viewing angles, poor lighting, far distance, and other impoverished viewing conditions. Critically, however, this accuracy comes at a cost of longer time to respond (Bülthoff, Edelman, \& Tarr, 1995; Kosslyn et al., 1994; Palmer, Rosch, \& Chase, 1981; Schendan \& Ganis, 2015; Srinivas, 1995; Tarr \& Pinker, 1989).

How can the brain achieve the perceptual constancy of cognition when visual input poorly matches memory? The MUSI account hypothesizes that later states 2 and M support the ability to categorize objects and recognize them as familiar under widely varying sensory conditions -- that is, the object constancy of memory (i.e., similar responses to particular objects despite changes in orientation, size, or other perceptual properties). This is important because, to be most widely utilized, memory or the meaning of a concept (e.g., 'dog') should be activated despite widely varying stimuli, such as its name and different perceptual instantiations across sensory modalities (e.g., pictures of dogs under diverse visual conditions, its sound, how it feels). As mentioned, critically, performance is slower when a stimulus provides impoverished information, as under ambiguous or nonoptimal sensory conditions (e.g., unusual viewpoints, unfamiliar instantiations, in fog, crowding, ). One reason for this slowing is that impoverished images match more poorly to representations in memory, potentially requiring further processing for an accurate solution to the perceptual problem. Further, feature dissimilarity can affect the construction of diagnostic cues used to decide the match of the perceived object to memory (Schyns, 1998). With impoverished images, computational modeling indicates that initial fast bottom-up processing along the ventral visual hierarchy cannot explain human performance levels (Serre, Oliva, \& Poggio, 2007). Instead, to do so, theories of perceptual hypothesis testing (PHT) implicate top-down feedback contributions (e.g., Gosselin \& Schyns, 2001; Grossberg, 1999; Hochstein \& Ahissar, 2002; Humphreys, Price, \& Riddoch, 1999; T. S. Lee \& Mumford, 2003; J. Liu et al., 2011; Lowe, 1985; Lowe, 2000; Ullman, 1995).

\subsection{Memory}

\subsubsection{Multiple memory systems theory}


MUSI describes how different kinds of memory are associated with different brain states and how these memory processes support visual cognition, so, next, memory theory is summarized; note, debates about memory theories are independent of MUSI but do inform it, and MUSI favors an embodied cognition framework. According to the multiple memory systems theory different kinds of memory depend on different brain systems (Schacter \& Tulving, 1994; Squire \& Zola-Morgan, 1991). Convergent evidence for dissociations between different kinds of memory has divided a system for 'explicit memory' that occurs with conscious awareness into 2 systems (Kan, Alexander, \& Verfaellie, 2009; Rosenbaum et al., 2005; Tulving, 1972; Vargha-Khadem, Gadian, \& Mishkin, 2001; Vargha-Khadem et al., 1997). (1) Episodic memory is tied to personallyexperienced events, and depends upon the mediotemporal lobe (MTL) (Eichenbaum, 2000). For example, episodic memory supports free recall (e.g., of objects on a study list) and recognition of an object from previous experience with it, including feelings of familiarity. (2) Knowledge memory supports world facts accumulated over a life time that are not tied to a personal episode, including 'semantic memory' about the meaning of words and objects and perceptual representation systems (e.g., visual object structure) (Warrington, 1982). (3) The knowledge system also supports semantic, conceptual, and knowledge-based perceptual implicit memory, which occurs without conscious effort to retrieve memory. This supports priming, which is measured behaviorally as performance differences, typically decreases in response time (RT) or increases in accuracy for repeated relative to new items, and measured neurally as activity differences between these conditions (Gabrieli, Fleischman, Keane, Reminger, \& et al., 1995; Gabrieli et al., 1994; Keane, Gabrieli, Mapstone, Johnson, \& Corkin, 1995; Keane, Gabrieli, Fennema, Growdon, \& Corkin, 1991; Tulving \& Schacter, 1990; Verfaellie, Cermak, Blackford, \& Weiss, 1990).

The MUSI account hypothesizes that, while priming can have small effects during state 1 under limited, extreme conditions, most types of knowledge and implicit memory occur typically and primarily in state 2 , and episodic memory occurs primarily in state $\mathrm{M}$. Research on knowledge has focused on the organization of meaningful (semantic) representations, especially in the context of language, but less work has been done on how perceptual knowledge is matched to stimulus information in order to categorize an object (e.g., how shape activates meaning). The MUSI account addresses this gap by focusing on perceptual knowledge.

\subsubsection{Amodal Knowledge in Anterior Temporal Lobe (ATL)}

The knowledge system, especially semantic memory, in multiple memory systems theory aligns with the standard theory of cognition: Conceptual knowledge is represented in a single amodal system (Tulving, 1972) that represents meaning in a symbol system, which has a uniform architecture and resides apart from modal systems for processing specific types of information, such as sensory, motor or emotional information (Fodor, 1983). An amodal system, if it exists, may reside in the ATL ( Visser, Jefferies, \& Lambon Ralph, 2010), which is widely accepted to support semantic memory, though the precise role is debated (Binney, Embleton, Jefferies, Parker, \& Ralph, 2010; Gorno-Tempini et al., 2004; A. C. Lee et al., 2006; Mahon \& Caramazza, 2009; Murray, Bussey, \& Saksida, 2007; Patterson, Nestor, \& Rogers, 2007; Rogers \& Patterson, 2007; Simmons \& Martin, 2009; K. I. Taylor, Moss, Stamatakis, \& Tyler, 2006).

\subsubsection{Embodied Memory in Modal Cortex}

However, some evidence invalidates strong versions of the standard theory, leading current views of knowledge to incorporate an alternative to a strong standard theory: embodied (or grounded) cognition theory that proposes all knowledge depends upon multiple modality-specific systems for sensorimotor "properties", as in perceptual systems based on the senses (e.g., vision), 
action systems for motor planning, emotional and mental state systems (Barsalou, 2008; Chao, Haxby, \& Martin, 1999; Devlin, Gonnerman, Andersen, \& Seidenberg, 1998; Dilkina, McClelland, \& Plaut, 2008; Downing, Chan, Peelen, Dodds, \& Kanwisher, 2006; Goldrick, 2008; Kable \& Chatterjee, 2006; A. Martin, 2007; McClelland \& Rogers, 2003; Moore \& Price, 1999; Paivio, 1991; Tyler \& Moss, 2001). Different systems support modality-specific, object, word, and face knowledge (Barsalou, 2008); for example, object-sensitive occipitotemporal cortex supports visual object knowledge. The main proposed mechanism for how cognition and memory, including knowledge and meaning, are grounded or embodied in modal information processing is mental simulation, which can be automatic or strategic (Barsalou, 2008, 2009; Glenberg, 1997). Simulation occurs when neural activity in modal association cortex triggers time-locked, recurrent and feedback activity across multiple lower-level modal processing areas, which are the areas from which the memory was initially constructed during the learning experience. Through this distributed multi-regional activity, seeing an object or reading its name (e.g., "dog") re-enacts associated features that were stored during earlier learning experiences (e.g. its shape, color, motion, actions with it), thereby constructing higher cognition, memory, and meaning. However, debates on the role of embodiment in cognition (Gerlach, 2007; Mahon \& Caramazza, 2008, 2009; Patterson et al., 2007) have motivated hybrid proposals, such as those that have both an amodal system and embodied knowledge (e.g., one amodal "hub"; multiple "convergence zones") (H. Damasio, Grabowski, Tranel, Hichwa, \& Damasio, 1996; Patterson et al., 2007). In an embodied cognition framework, MUSI hypothesizes that automatic mental simulation occurs in state 2, whereas strategic simulation occurs in state $\mathrm{M}$.

\section{State 1: Details and further evidence}

\subsection{Object processing along the visual pathways}

A key hypothesis of the MUSI account is that, under unambiguous or optimal stimulus conditions, processes during state 1 that underlie categorical perception may provide sufficient information to enable later states to compute a decision rapidly with minimal or no additional processing. In state 1, initial activation of object processing cortex feeds forward from occipital to anterior temporal cortex along the ventral stream and from occipital into posterior parietal cortex along the dorsal stream; note, as the feedforward sweep proceeds, this activity automatically triggers reflexive feedback inputs from higher to lower areas, as well as between dorsal and ventral pathways (Bacon-Mace, Mace, Fabre-Thorpe, \& Thorpe, 2005; Foxe \& Simpson, 2002; Hupe et al., 2001; Hupe et al., 1998; Keysers \& Perrett, 2002; Rennie, Robinson, \& Wright, 2002), and the ventral pathway processes of interest can be influenced by the dorsal visual pathway, which processes information more rapidly (Bar, 2003).

Like visual cortex in nonhuman primates (Baker, Behrmann, \& Olson, 2002; Connor, Brincat, \& Pasupathy, 2007; Felleman \& Van Essen, 1991; N. Li \& DiCarlo, 2008; Olson, 2001; K. Tanaka, 2003), human occipitotemporal cortex shows progressive hierarchical convergence as processing feeds forward from posterior to anterior areas. Along this feedforward pathway, stimulus selectivity becomes increasingly complex from more elementary, local features (e.g., shape or form, color, spatial location, and motion) and greater visual-specificity to higher-order global shapes and combinations of features and increasing visual constancy (Eger, Schyns, \& Kleinschmidt, 2004; Grill-Spector et al., 1999; Grill-Spector \& Malach, 2004; Hasson, Levy, Behrmann, Hendler, \& Malach, 2002; Lerner, Hendler, Ben-Bashat, Harel, \& Malach, 2001; Levy, Hasson, Avidan, Hendler, \& Malach, 2001; Op de Beeck, Haushofer, \& Kanwisher, 2008; K. Tanaka, Saito, Fukada, \& Moriya, 1991; Tjan, Lestou, \& Kourtzi, 2006). The fast feedforward 
sweep through the posterior ventral cortical hierarchy progresses from primary visual cortex in area V1 then to V2 then to V4 and into inferotemporal cortex in nonhuman primates. This wellestablished hierarchical organization of visual cortex has inspired bottom-up theories of cognition that propose how this feedforward sweep can achieve a category decision (Riesenhuber \& Poggio, 1999; Serre, Wolf, Bileschi, Riesenhuber, \& Poggio, 2007; Thorpe, Fize, \& Marlot, 1996).

The functional organization of occipitotemporal cortex is such that there is systematic variation in cortical location according to the category of the visual image, and so, in the MUSI account, different categories of objects have states that are similar functionally but differ in cortical organization and associated spatiotemporal dynamics (e.g., Schendan \& Ganis, 2012). Some areas are specialized for processing broad domains of objects, such as faces, buildings, and body parts (Downing et al., 2006), but categorical organization is experience-dependent (Bukach, Gauthier, \& Tarr, 2006; Folstein, Palmeri, Van Gulick, \& Gauthier, 2015; Wong, Palmeri, Rogers, Gore, \& Gauthier, 2009). In humans, like earlier areas, occipitotemporal cortex is retinotopic, such that adjacent locations in the retina (and corresponding visual field) are represented in adjacent neurons in the cortex. However, at this advanced processing stage, occipitotemporal cortex has a categoryspecific retinotopic organization such that objects requiring central vision (e.g., faces and wordlike images) have center-biased cortical representations, while objects requiring integration across large spatial scales (e.g., buildings) are associated with periphery-biased representations, and objects requiring both central and peripheral vision (e.g., tools) have both central and peripheral representations (Hasson et al., 2002). Occipitotemporal cortex also responds more strongly to images containing objects or object-like structures, including scenes of multiple discernable objects, than images with no coherent object structure in which no figure is discernable (Malach et al., 1995): This 'object-sensitivity' is a hallmark of this cortex, which is necessary for normal behavior on a wide range of tasks with objects. For example, patients with occipitotemporal dysfunction have visual agnosia: impaired perceiving, categorizing, and recognizing of visual objects with the pattern of deficits varying with the locus of damage (Behrmann, Peterson, Moscovitch, \& Suzuki, 2006; Farah, 1990; Humphreys et al., 1999; Riddoch \& Humphreys, 1987; Warrington, 1982).

Across object-sensitive occipitotemporal cortex, in addition to the center-periphery retinotopic organization, the functional cortical architecture is widely distributed and overlapping across category-specific (e.g., face) and general (nonface) areas (Gauthier, Curran, Curby, \& Collins, 2003; Gauthier, Tarr, Anderson, Skudlarski, \& Gore, 1999; Kanwisher, McDermott, \& Chun, 1997; Kriegeskorte, Mur, \& Bandettini, 2008; Kriegeskorte, Mur, Ruff, et al., 2008; Op de Beeck, Haushofer, et al., 2008; Schwarzlose, Baker, \& Kanwisher, 2005; Yovel \& Kanwisher, 2004). Such distributed organization seems to depend substantially on selectivity for visual features (e.g., color, shape, texture, motion, location, size) and visual similarity among objects, which structures perceptual processing and decision-making (Cichy, Kriegeskorte, Jozwik, van den Bosch, \& Charest, 2019; Grill-Spector et al., 1999; Jozwik, Kriegeskorte, \& Mur, 2016; Kourtzi \& Kanwisher, 2001; Op de Beeck, Deutsch, Vanduffel, Kanwisher, \& Dicarlo, 2007, 2008; Op de Beeck, Wagemans, \& Vogels, 2008). For example, objects within a category tend to be more visually similar to each other than objects from another category, and activity patterns across occipitotemporal cortex show an organization such that objects within a category (e.g., shoes) or a feature (e.g., color) activate a similar set of regions that is distinct but somewhat overlapping with the set of regions activated by a different category (e.g., chairs) or feature (e.g., shape) (e.g., Cichy et al., 2019; Haxby et al., 2001). 


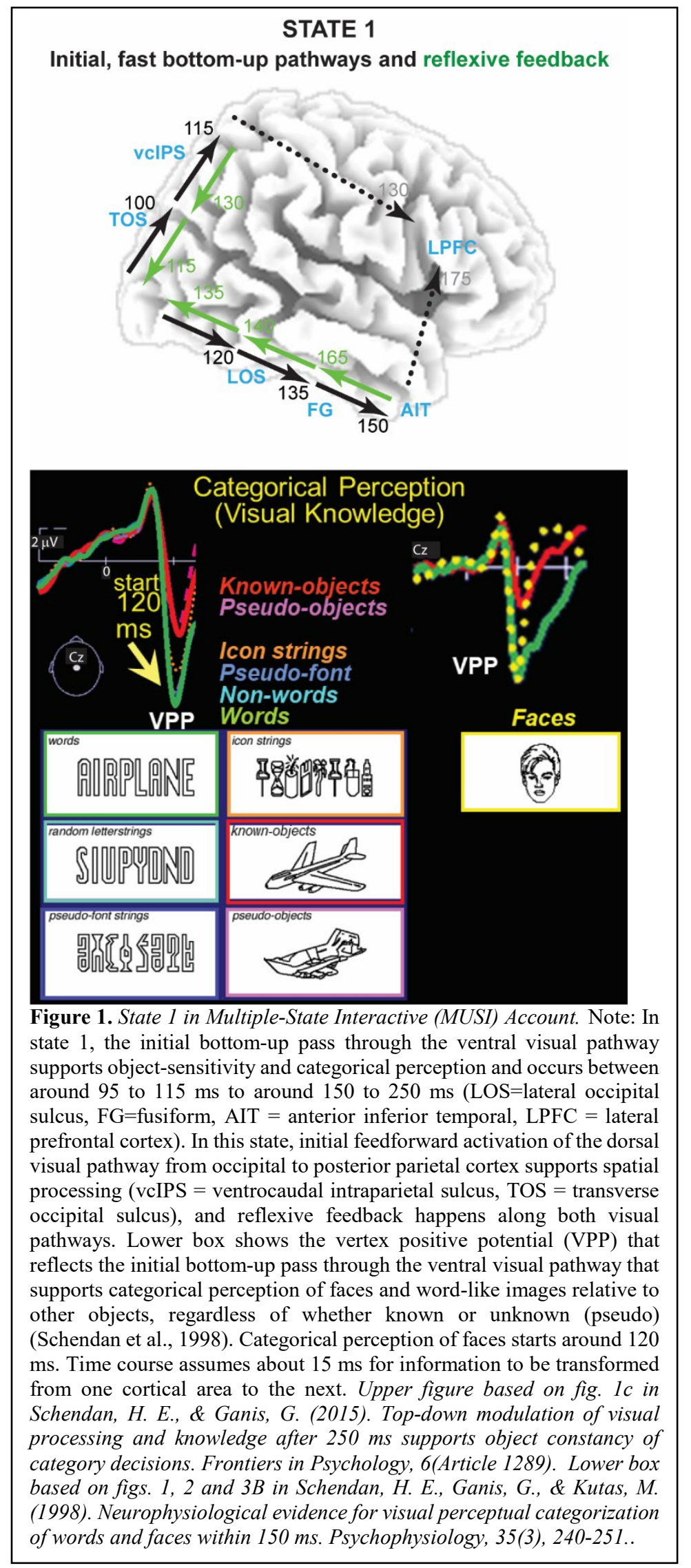




\subsection{Time course of visual object processing within $250 \mathrm{~ms}$ in state 1}

In state 1, predominantly bottom-up processes along the visual pathways occurring within $250 \mathrm{~m}$ in humans support object-sensitivity, perceptual grouping and activation of knowledge for categorical perception, implicit learning and implicit memory. Neurophysiological and computational evidence indicates that the initial, fast bottom-up activation of higher-order extrastriate visual areas happens from around $95 \mathrm{~ms}$ until around $\sim 170$ to $250 \mathrm{~ms}$ (David et al., 2006; Schendan \& Lucia, 2010). The early P1(00) from 95 to $115 \mathrm{~ms}$ indexes occipitoparietal cortex along the dorsal stream for processing spatial information, location, motion, and functional knowledge about objects, and the late P1(00) from 120 to $140 \mathrm{~ms}$ indexes occipitotemporal cortex along the ventral stream for processing the shape and color of objects (Di Russo, Martinez, Sereno, Pitzalis, \& Hillyard, 2001).

\subsubsection{Object-sensitivity and categorical perception within $180 \mathrm{~ms}$}

The initial, fast bottom-up activation of human object-sensitive cortex takes place from around 95 ms until around 155 ms (Craddock, Martinovic, \& Muller, 2015; Schendan \& Lucia, 2010). Human occipitoparietal cortex along the dorsal visual pathway and occipitotemporal cortex along the ventral visual pathway have been shown to be object-sensitive using standard methods in fMRI, for example, comparing images of real objects and completely phase scrambled versions of them (Schendan \& Stern, 2007). ERPs using this fMRI paradigm reveals that the P1(00) (in a large group of people, $N=57$ ) first begins to differ between the real objects and phase scrambled versions (i.e., shows object-sensitivity) around $95 \mathrm{~ms}$, reflecting processes to achieve figureground segregation (Schendan \& Lucia, 2010). This object-sensitive neurophysiological activity localizes especially to the right hemisphere in extrastriate occipital cortex in Brodmann's area (BA 18) during the early P1 (95-115 ms) and inferior occipital gyrus in BA 18 during the late P1 (120$140 \mathrm{~ms}$ ) and lasts until around $155 \mathrm{~ms}$. Furthermore, these areas show reactivation after $200 \mathrm{~ms}$, consistent with the MUSI account of multiple states of activation of the same region. Thus the early P1(00) shows the earliest object-sensitivity, which is a hallmark of visual cortical processing of object structure and defines the core object processing areas in fMRI (Schendan \& Stern, 2007). However, categorical perception does not yet occur during the P1(00) (Ganis, Smith, \& Schendan, 2012).

Instead, the initial bottom-up activation of visual object knowledge in human occipitotemporal areas that supports the earliest categorical perception is revealed in a vertex positive peak (VPP), a frontocentral positivity peaking around 150-170 ms, and its polarityinverted counterpart at occipitotemporal locations, the N170 (Allison, Puce, Spencer, \& McCarthy, 1999; David et al., 2006; Joyce \& Rossion, 2005; Schendan, Ganis, \& Kutas, 1998). From between 120 and $\sim 170$ to 200 ms post-stimulus, the VPP/N170, and its magnetoencephalographic (M170]) and intracranial equivalents (N200s and their polarity-inverted P180s) discriminates globally between coarse, behaviorally-relevant object categories, such as a face versus a wide variety of non-face objects (Ganis \& Schendan, 2008; Ganis et al., 2012; Schendan \& Ganis, 2013; Schendan et al., 1998). The VPP/N170 indexes categorical perception based on expertise with particular image categories, being larger for faces and letter-strings (e.g., words) than other objects, even when perceptually-matched (Allison, McCarthy, Nobre, Puce, \& Belger, 1994; Allison et al., 1999; Bentin, Allison, Puce, Perez, \& McCarthy, 1996; Ganis \& Schendan, 2008; Halgren et al., 1995; Joyce \& Rossion, 2005; McCarthy, Puce, Belger, \& Allison, 1999; Puce, Allison, \& McCarthy, 1999). Spatial configuration and salient face features affect VPP/N170 amplitude and face cognition (Bentin, Golland, Flevaris, Robertson, \& Moscovitch, 2006; Flevaris, Robertson, \& Bentin, 2008; Gu, Li, Yang, \& Zhu, 2007). The VPP/N170 category-specificity has been shown 
primarily for faces and words (Schendan et al., 1998), and other objects -- often one category like cars -- were tested mainly for comparison (Itier \& Taylor, 2004b; Rossion, Joyce, Cottrell, \& Tarr, 2003). Critically, face-specificity holds when comparing faces with a wide variety of object categories and cannot be explained by perceptual interstimulus variance (i.e., faces vary less from each other perceptually than do other objects, e.g., cars) (Ganis et al., 2012; Schendan \& Ganis, 2013). Neural processes underlying VPP/N170 have been implicated in learning and representing knowledge for expert object categorization (Curran, Tanaka, \& Weiskopf, 2002; Rossion, Curran, \& Gauthier, 2002; Rossion, Kung, \& Tarr, 2004; Scott, Tanaka, Sheinberg, \& Curran, 2006; J. W. Tanaka \& Curran, 2001). Convergent evidence localizes the brain sources to occipitotemporal cortex for objects and to face-specific areas in posterior fusiform gyrus, inferior occipital gyrus, and superior temporal sulcus for faces (Bötzel, Schulze, \& Stodieck, 1995; Corrigan et al., 2009; Horovitz, Rossion, Skudlarski, \& Gore, 2004; Itier \& Taylor, 2004c; Jacques et al., 2019; Miki, Watanabe, Kakigi, \& Puce, 2004; Puce et al., 1999; Rossion et al., 1999; Schendan et al., 1998; Schweinberger, Pickering, Jentzsch, Burton, \& Kaufmann, 2002; Watanabe, Kakigi, \& Puce, 2003). Computational modelling indicates that feedforward processing from V1 to higher areas along the ventral visual pathway explains the category-specificity of the VPP/N170 (David et al., 2006). This provides critical evidence for the MUSI idea that predominantly feedforward processing and reflexive feedback and recurrent processing during state 1 supports categorical perception.

The VPP/N170 also shows object-sensitivity, a hallmark of visual cortical processing of object structure. The VPP/N170 shows object-sensitivity that localizes to posterior fusiform gyrus in BA 37 between about 115 and $180 \mathrm{~ms}$ (Schendan \& Lucia, 2010). This effect localizes to the occipitotemporal areas that are object-sensitive in fMRI (Schendan \& Lucia, 2010; Schendan \& Stern, 2007), and intracranial N200s show object-sensitive effects (Allison et al., 1999; Horovitz et al., 2004; Malach et al., 1995; Rousselet, Husk, Bennett, \& Sekuler, 2008). Crucially, objectsensitive and categorical perception effects on the VPP/N170 consistently start around $120 \mathrm{~ms}$ post-stimulus onset (Ganis et al., 2012; Schendan \& Ganis, 2013; Schendan et al., 1998; Schendan \& Lucia, 2010), defining the start time for the fast bottom-up pass through human object-sensitive occipitotemporal areas that supports the earliest visual object knowledge activation for categorical perception.

\subsubsection{Limited Object Constancy}

Evidence suggests that state 1 has limited object constancy, and, instead, processes important for object constancy occur after state 1. First, object constancy entails visually invariant (or tolerant) responses, but the VPP/N170 to faces modulates with changes in visual properties like inversion, eccentricity, size, resolution, and spatial position (Caharel, d'Arripe, Ramon, Jacques, \& Rossion, 2009; Kovacs, Zimmer, Harza, Antal, \& Vidnyanszky, 2005; Kovacs, Zimmer, Harza, \& Vidnyanszky, 2007; Mercure, Dick, Halit, Kaufman, \& Johnson, 2008; Rousselet, Husk, Bennett, \& Sekuler, 2005), showing limited object constancy. Second, bottom-up accounts of object cognition predict less neural activity for more (vs. less) impoverished objects along the ventral visual hierarchy because more impoverished images have fewer visual features and so activate fewer neurons and/or activate each neuron more weakly than less impoverished images. Indeed, by $120 \mathrm{~ms}$, VPP/N170 amplitude is smaller for impoverished or scrambled than for intact houses, faces and other body parts (Horovitz et al., 2004; Jemel et al., 2003; Rousselet et al., 2008; Thierry et al., 2006). N170 impoverishment effects correlate with occipitotemporal fMRI signal (Horovitz et al., 2004), but the fMRI signal in object-sensitive areas is instead higher for impoverished than intact images (not smaller like the VPP/N170) and for more than less 
impoverished images (Ganis et al., 2007; Schendan \& Stern, 2008), as discussed more under state 2. This suggests that the VPP/N170, but not the fMRI, effects reflect predominantly the initial fast bottom-up pass in state 1, which also shows limited object constancy (i.e., limited ability to compensate for image impoverishment); caveat, more versus less activity does not map directly to more versus less neural activity using electromagnetic measures, ERPs reflect phase-locked activity (Nunez \& Srinivasan, 2006) while fMRI does not, and the relation between electromagnetic measures and fMRI is not straightforward with evidence suggesting they reveal different, complementary information (Itthipuripat, Sprague, \& Serences, 2019).

\subsubsection{Perceptual Grouping and Implicit Learning and Memory within $250 \mathrm{~ms}$}

Further evidence that state 1 reflects predominantly bottom-up perceptual computations comes from evidence that an occipitotemporal P2(00) between 150 and $250 \mathrm{~ms}$ indexes the Gestalt perceptual grouping process of good continuation and closure (Schendan \& Lucia, 2010), and implicit memory for these perceptual processes can transfer from a study experience to a memory test (Schendan \& Kutas, 2007a, 2007b). The P200 peaks later than the P1(00) and VPP/N170, consistent with P200 grouping processes capitalizing on earlier figure-ground segregation and categorical perception. Perceptual grouping can entail automatic recurrent and feedback processes along the visual pathway (Lamme \& Roelfsema, 2000), consistent with the role of reflexive feedback in grouping during state 1.

Overall, the ERP evidence indicates that object-related perceptual processing in state 1 occurs between $95 \mathrm{~ms}$ (starting with the P100) and $250 \mathrm{~ms}$ (during the P200). Regarding memory, knowledge for categorical perception occurs between 120 and 170 to $200 \mathrm{~ms}$ during the VPP/N170 (Schendan \& Ganis, 2013), implicit learning can also modulate the VPP/N170 (Scott et al., 2006), and implicit memory for perceptual grouping processing can occur as early as 150 to $250 \mathrm{~ms}$ during the P200 (Schendan \& Kutas, 2007b).

\subsubsection{Limited Role in Higher Cognition}

Thus, state 1 supports detecting salient shapes against a background (object-sensitivity), categorical perception, perceptual grouping, and perceptual implicit learning and memory, but what about higher cognitive processes that compute a decision? Decision making is a core function of cognition (Gold \& Shadlen, 2007), and visual cognition and decision making accounts have so far emphasized processing in state 1 before $200 \mathrm{~ms}$ for supporting cognition, with some emphasizing bottom-up processing (e.g., Philiastides \& Sajda, 2007; Riesenhuber \& Poggio, 1999; Serre, Oliva, et al., 2007; Thorpe et al., 1996) and some positing a role for feedback inputs (e.g., Bar, 2003). However, convergent evidence indicates that, while state 1 contributes information to decisions, these early processes do not compute the decision (Allison et al., 1999; Bentin et al., 1996; David et al., 2006; Minamimoto, Saunders, \& Richmond, 2010; Rossion \& Jacques, 2008; Schendan et al., 1998; Schendan \& Lucia, 2010; Warrington, 1982).

For example, if state 1 computes the decision, then the processes that make up this state should vary with task. To the contrary, the VPP/N170 is largely task-independent (e.g., Cauquil, Edmonds, \& Taylor, 2000; Crist, Wu, Karp, \& Woldorff, 2007; Ganis \& Schendan, 2008; Guillaume \& Tiberghien, 2001; Joyce, Schyns, Gosselin, Cottrell, \& Rossion, 2005; MouchetantRostaing, Giard, Bentin, Aguera, \& Pernier, 2000; Philiastides \& Sajda, 2006; Rousselet, Mace, \& Fabre-Thorpe, 2004; Schendan et al., 1998; Smith, Gosselin, \& Schyns, 2004). For example, the task goal affects the VPP/N170 minimally, if at all, but has much larger and consistent effects on later ERPs after $\sim 200 \mathrm{~ms}$ in response to objects and faces (Philiastides, Ratcliff, \& Sajda, 2006; for review see Rousselet, Gaspar, Wieczorek, \& Pernet, 2011; Schendan \& Ganis, 2012; e.g., Stahl, Wiese, \& Schweinberger, 2010). Indeed, even some extreme task manipulations can have no 
detectable effect on ERPs in state 1; for example, no differences in N170 categorical perception effects despite wide variations in task difficulty (e.g., detection of color vs. detection of immediate repetition vs. detection of a fruit/vegetable category, Schendan et al., 1998). Where there are effects in both states 1 and 2, effects can also differ in pattern (Voss, Schendan, \& Paller, 2010). Such findings also provide evidence dissociating states 1 and 2, and further evidence for this includes findings that task, mental imagery, memory and attention effects are much larger on later ERPs in state 2 than earlier ERPs in state 1 (Schendan \& Ganis, 2012, 2015).

Moreover, if the VPP/N170 were indexing processes underlying higher cognition and decisions, factors affecting cognitive and decision performance should modulate it strongly. To the contrary, the VPP/N170 is typically not modulated by categorization success, and shows shortterm adaptation (habituation) and little or no long-term repetition effects related to associative memory (Ganis \& Schendan, 2008; Gruber \& Muller, 2006; A. Harris \& Nakayama, 2007, 2008; Heisz, Watter, \& Shedden, 2006; Henson \& Rugg, 2003; Henson, Rylands, Ross, Vuilleumeir, \& Rugg, 2004; Itier \& Taylor, 2004a; Jeffreys, 1996; Martens, Schweinberger, Kiefer, \& Burton, 2006; Schendan et al., 1998; Schendan \& Kutas, 2002, 2003, 2007a, 2007b; Schendan \& Maher, 2009; Squire \& Zola-Morgan, 1991). Further, tasks that require top-down feedback processes, cognitive control and endogenous attention can modulate the VPP/N170 (Ganis \& Schendan, 2008, 2011), but these early effects are much smaller than effects on later ERPs after $200 \mathrm{~ms}$ (Schendan \& Ganis, 2012). For example, the VPP/N170 is much reduced by adaptation from a recently perceived stimulus (a bottom-up process) but slightly enhanced by sustained visual mental imagery, involving entirely top-down modulation from the frontal lobe (Ganis \& Schendan, 2008). However, later ERPs show different patterns and much larger effects (Schendan \& Ganis, 2012).

In addition, major cognitive factors (e.g., mental imagery, category decision success, meaning, semantic context) modulate occipitotemporal cortex sensitively in fMRI studies, as well as later ERPs after $200 \mathrm{~ms}$, but do not likewise affect the VPP/N170 (e.g., Ganis \& Kutas, 2003; Schendan \& Lucia, 2009; Schendan \& Lucia, 2010; Schendan \& Maher, 2009). These minimal effects on the VPP/N170 stand in contrast with many studies showing robust fMRI effects in occipitotemporal cortex with such manipulations (de Fockert, Rees, Frith, \& Lavie, 2004; de Fockert, Rees, Frith, \& Lavie, 2001; DeGutis \& D'Esposito, 2007; Ganis, Thompson, \& Kosslyn, 2004; Henson et al., 2003; Pins, Meyer, Foucher, Humphreys, \& Boucart, 2004; Schendan \& Stern, 2007, 2008; Stern et al., 1996). Instead, cognitive and task manipulations strongly affect fMRI signal and later ERPs (Guillaume \& Tiberghien, 2001).

\section{State 2: Details and further evidence}

Accounts of cognition and decisions have focused on the initial fast bottom-up sweep with unambiguous sensory information (Philiastides et al., 2006; Philiastides \& Sajda, 2007; Ratcliff, Philiastides, \& Sajda, 2009; Riesenhuber \& Poggio, 1999; Serre, Oliva, et al., 2007), but the MUSI account proposes that higher cognition, including decision-making, occurs for the first time in state 2 . In this state, starting between $\sim 150-230 \mathrm{~ms}$ and lasting until around $500 \mathrm{~ms}$ under common conditions, sustained interactive activation of object processing areas in occipitotemporal cortex involves top-down, recurrent, and bottom-up processes among these areas and with occipitoparietal cortex, and, most critically, with prefrontal cortex (PFC). A central function of this interactive activation is to accumulate evidence (e.g., perceptual information, memory) for a decision or task goal (David et al., 2005; Fazl et al., 2009; Philiastides \& Sajda, 2007; Rousselet et al., 2011; Schendan \& Kutas, 2002, 2003, 2007a; Schendan \& Maher, 2009; Schweinberger, Kaufmann, Moratti, Keil, \& Burton, 2007; Ullman, 1996). MUSI and related accounts propose 
that higher-order or complex cognition, decisions, fine discriminations, and awareness (e.g., phenomenological consciousness) require additional neural computations involving later local recurrent, feedback and inter-areal contributions to perceptual information processing (Bradski \& Grossberg, 1995; David et al., 2005; Enns \& Lleras, 2008; Grossberg, 1999; Hochstein \& Ahissar, 2002; Lamme, 2003; Lamme \& Roelfsema, 2000; T. S. Lee, Yang, Romero, \& Mumford, 2002; Lowe, 2000; Mumford, 1992; Treisman, 2006; Ullman, 1995; Yang, Tarr, Kass, \& Aminoff, 2019). The initial feedforward sweep through the ventral visual stream does not, itself, compute the actual target or category decision. Instead, based on the information and, presumably, knowledge activated during the initial feedforward sweep, later decision making processes use this information and knowledge to compute the decision in state 2.

A hallmark of state 2 is that this is the first time when lateral PFC (LPFC) modulates posterior processing according to the current behavioral context and goal in order to compute an appropriate mental or behavioral response. Human LPFC has a hierarchical organization of increasingly abstract processing from more caudal to more rostral parts to achieve a mental or overt action (Badre, 2008; Badre \& D'Esposito, 2007; Badre, Hoffman, Cooney, \& D'Esposito, 2009; Buckner, 2003; Bunge, 2004; Courtney, 2004; Fuster, 2003; Graziano \& Aflalo, 2007; O'Reilly, Noelle, Braver, \& Cohen, 2002; Petrides, 2005; Postle, 2006; Stern et al., 2000). This system maintains patterns of activity for multiple types of information (e.g., linguistic, visuospatial, object, rules) in functionally distinct neural populations, each of which influences (controls) other areas like occipitotemporal cortex (Bunge, 2004; T. Liu, Pestilli, \& Carrasco, 2005; Montojo \& Courtney, 2008; Sayala, Sala, \& Courtney, 2006). The MUSI account proposes that these later neural computations support selection of information, memory or knowledge for a decision. These state 2 processes are especially important when (a) the task is open-ended (e.g., confrontation naming), (b) basic/entry level (e.g., dog) or even more precise (subordinate level, e.g., collie) access to meaning is necessary, (c) the target has not been pre-cued or specified, or (d) sensory input is less similar to stored knowledge, as when stimuli are impoverished or novel, especially regarding the spatial configuration of features. The task, memory, mental imagery, and attentional orienting in state 2 strongly affect (much more than in state 1) which posterior areas are activated, the level of activation, and how areas interact. Further, the pattern of effects can differ depending upon the particular posterior cortical areas involved, such as face versus object areas (Ganis \& Schendan, 2008; Schendan \& Ganis, 2012). The MUSI account is consistent with interactive activation and competition models of object naming that posit bidirectional flow among network nodes for visual object knowledge, semantic knowledge, and name knowledge and another node for the decision (Humphreys et al., 1999; Noppeney, Price, Penny, \& Friston, 2006). The MUSI account also proposes that State 2 reflects knowledge, meaning, and implicit memory processes. Next, further evidence for these proposals is reviewed.

The MUSI account hypothesizes that state 2 processes are indexed by mid-latency negative ERPs, such as the frontal N2(00) related to cognitive control, frontal N3(00) complex related to visual object processing, and the centroparietal N400 related to semantic processing. This is consistent with similar proposals that, following initial bottom-up perceptual processing (or stimulus registration) during the $\mathrm{P} 1$ and VPP/N170, mid-latency ERPs reflect the earliest times when cognitive control and decision-making processes operate, including stimulus selection, information and knowledge retrieval, and categorization functions (Folstein \& Van Petten, 2008; Ritter et al., 1979). 
STATE 2 ACTIVE TASK NETWORK

Decision, Model Selection \& Perceptual Hypothesis Testing (PHT)

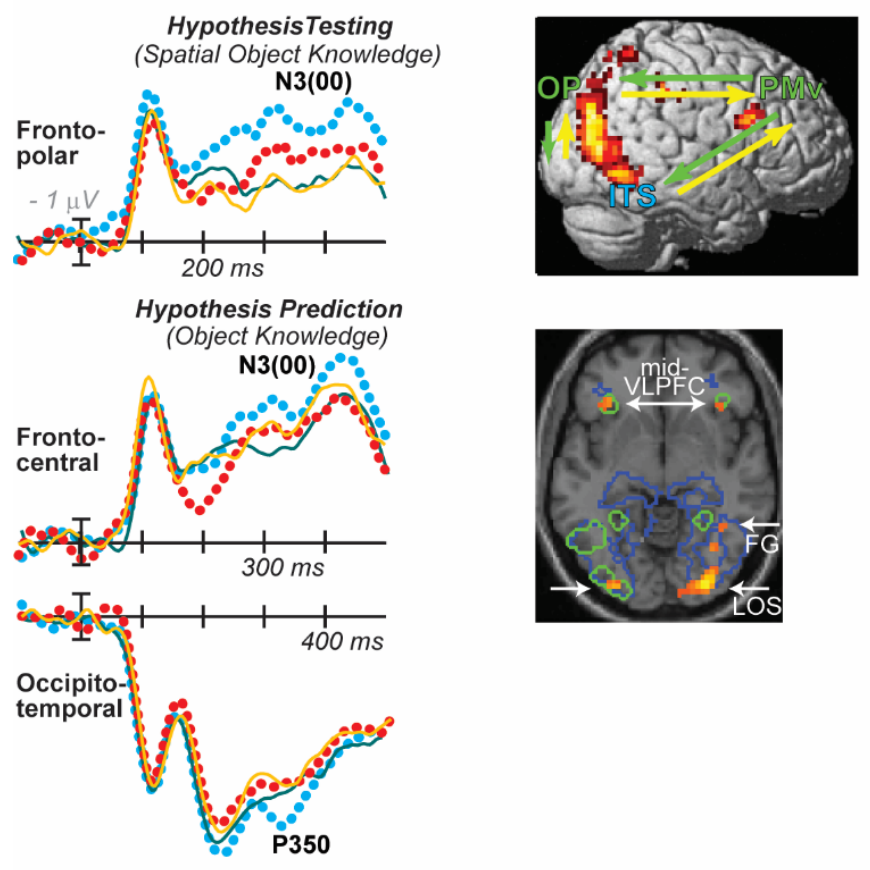

STATE M MULTIPLE NETWORK (predominantly DEFAULT MODE for Internal Evaluation)
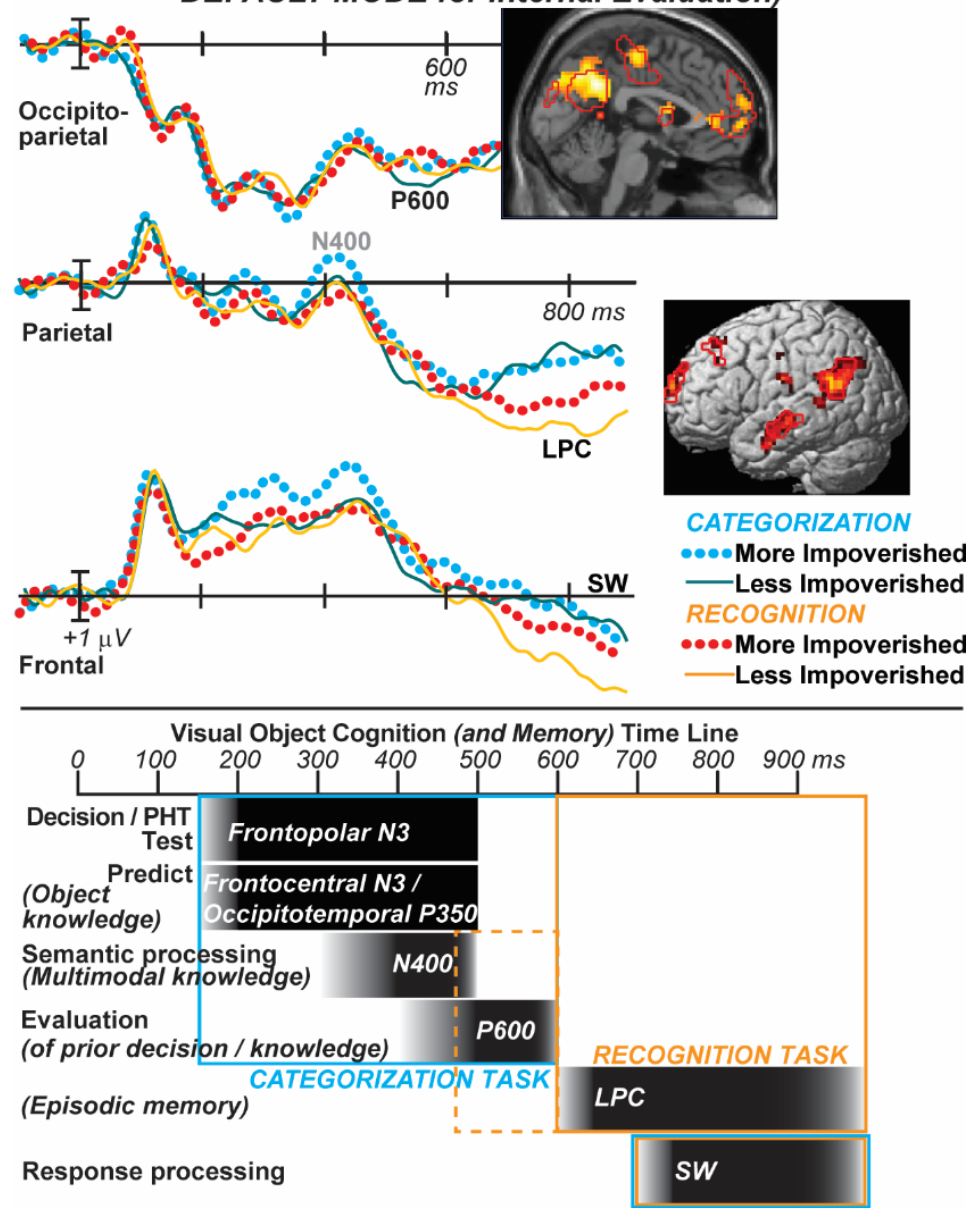

Figure 2. States 2 and M in Multiple-State Interactive (MUSI) Account. Note: Statistical parametric maps on canonical anatomical brains modified from an fMRI study (Schendan \& Stern, 2008). Time line (bottom) summarizes the timing of state 2 and M processes and their ERP markers for category decision processes, between about 150-230 ms and lasting until around $500 \mathrm{~ms}$ or later in state 2 , and after $500 \mathrm{~ms}$ for evaluation processes and after about $600-700 \mathrm{~ms}$ for episodic recognition processes in state M. STATE 2: Frontopolar N3 shows task-independent impoverishment effects. Likewise for $\mathrm{fMRI}$, the testing system $(\mathrm{PMv}=$ caudal ventrolateral prefrontal and ventral premotor cortex (BA 44/6), OP = occipitoparietal cortex, ITS=inferotemporal sulcus) in the active task network is more active for more than less impoverished real objects on both categorization and recognition tasks. Frontocentral N3 shows categorization-specific impoverishment effects. Likewise for $\mathrm{fMRI}$, the prediction system (mid-VLPFC $=$ midventrolateral prefrontal cortex [BA 47/12], LOS=lateral occipital sulcus, $\mathrm{FG}=$ fusiform) in the active task network is more active for more than less impoverished real objects on categorization more than recognition. A centroparietal N400 index of abstract semantic memory also shows this pattern. State 2 processes may also support automatic simulation for grounding cognition in modal information processing. STATE M: Like the pattern in parts of the default mode network (medial cortex [retrosplenial, posterior cingulate, medial prefrontal], lateral inferior parietal, and middle temporal gyrus), occipitoparietal positivity from 500 to $600 \mathrm{~ms}$ on the P600 shows impoverishment effects on categorization, and, after $700 \mathrm{~ms}$, the late positive complex (LPC) is more positive for less than more impoverished images on recognition more than categorization. Also after $700 \mathrm{~ms}$, the frontal slow-wave (SW), reflecting response related activity or retrieval orientation, shows impoverishment effects (more positive for less than more impoverished images) only on recognition. These later State $M$ processes may also support episodic simulation for grounded cognition. Notably, a slow wave tentatively included in state $\mathrm{M}$ may better be characterized in a late response planning state. Figure based on ERP data from Schendan, H. E., Lucia, L. C., \& Bridges, D. (submitted). Visual Object Categorization and Episodic Recognition Tasks Recruit Common and Distinct Cortical Processes after 200 ms. Also fMRI figs. 3a, 6, 7 in Schendan, H. E., \& Stern, C. E. (2008). Where Vision Meets Memory: Prefrontal-Posterior Networks for Visual Object Constancy during Categorization and Recognition. Cerebral Cortex, 18(7), 1695-1711.

Bottom time line shows example time course of state 2 (N3/P3, N400) processes during a category decision task (blue box) and state M processes (P600, LPC, slow-wave) during an episodic recognition task (orange box). The N400-P600 may reflect a transition (orange dotted box) between states 2 and M, whereby semantic memory (e.g., for categorization) increasingly contributes to episodic memory (e.g., for recognition) and active task network activity in state 2 (that predominates for decision-making) transitions to increasing contributions of default mode network activity in state $\mathrm{M}$ (for internal evaluation, episodic memory, access consciousness and creativity). 


\subsection{N3(00) Complex: Cognition and decisions about objects}

The N3(00) complex is a negative-going set of ERP components that peak between 200 and $500 \mathrm{~ms}$, typically around 300 to $400 \mathrm{~ms}$, and localize to occipitotemporal cortex (Schendan \& Ganis, 2015; Schendan \& Maher, 2009; Sehatpour, Molholm, Javitt, \& Foxe, 2006; Sehatpour et al., 2008). The $\mathrm{N} 3$ has a scalp distribution with an anterior maximum (using references around the mastoid) or the components sometimes show a polarity inversion such that there are simultaneously negative-going waves anteriorly and positive-going waves posteriorly (e.g., a posterior P350 complex, especially using an anterior or common average reference) (Schendan et al., 1998; Schendan \& Kutas, 2003; Schendan \& Maher, 2009). This scalp distribution is similar to the VPP/N170 (Joyce \& Rossion, 2005) with similar implications for underlying brain sources in occipitotemporal cortex. MUSI includes some frontal N2(00) components that are sensitive to perceptual match and cognitive control between 200 and $350 \mathrm{~ms}$ (see for review Folstein \& Van Petten, 2008) as part of the N3 complex (Schendan \& Lucia, 2010; Schendan \& Maher, 2009).

Next, key evidence is summarized that the frontal N3(00) complex is the first ERP (and state 2 the first state) to reflect activity in occipitotemporal cortex related to the success of higher visual object cognition and decisions. Like the VPP/N170 (state 1) and fMRI activation, the N3 is objectsensitive, category-specific, and shows adaptation effects (Ganis \& Schendan, 2008; Schendan \& Ganis, 2012; Schendan \& Lucia, 2010). However, unlike state 1 but like occipitotemporal and ventral LPFC (VLPFC) activity captured in fMRI, the N3 varies dramatically with mental imagery and factors affecting category decision success, such as stimulus typicality and impoverishment, implicit memory, knowledge, and meaning (Ganis \& Kutas, 2003; Philiastides \& Sajda, 2007; Schendan \& Ganis, 2012; Schendan \& Kutas, 2002, 2003, 2007a; Schendan \& Lucia, 2009, 2010; Schendan \& Maher, 2009; Schendan \& Stern, 2008; Voss et al., 2010). Crucially, fMRI findings demonstrate that human occipitotemporal cortex shows similar patterns of modulation as the N3, and generally unlike the earlier VPP/N170 (Ganis et al., 2007; Schendan, submitted; Schendan \& Lucia, 2012; Schendan \& Stern, 2007, 2008). Further, intracranial ERPs between 200 and $400 \mathrm{~ms}$ implicate brain sources in VLPFC and occipitotemporal cortex for objects (Allison et al., 1999; Puce et al., 1999), and fMRI effects have been found in VLPFC, posterior parietal, and occipitotemporal cortex for objects in scenes (Gronau, Neta, \& Bar, 2008; Henderson, Larson, \& Zhu, 2007, 2008; Park, Intraub, Yi, Widders, \& Chun, 2007). Intracranial ERPs also indicate that (a) occipitotemporal modulation with categorization success occurs from 228 to $420 \mathrm{~ms}$ during state 2, not during earlier P1 and N170 potentials in state 1, (b) LPFC responds to visual objects around $200 \mathrm{~ms}$ (Allison et al., 1999), and (c) interactive activity between occipitotemporal and VLPFC regions occurs between 150 to $225 \mathrm{~ms}$ and 400 to $475 \mathrm{~ms}$ during state 2 (Sehatpour et al., 2008).

Most important, the N3 shows the earliest modulation with factors affecting category decision performance (Schendan \& Kutas, 2002; Schendan \& Maher, 2009). The N3 is less negative for correct than failed categorization, category members than nonmembers, more than less confidently categorized objects, faster than slower categorization, and categorization of objects with all features rather than missing one or more parts (Doniger et al., 2000; Folstein \& Van Petten, 2004; Gratton, Evans, \& Federmeier, 2009; Gruber \& Muller, 2006; Holcomb \& McPherson, 1994; Schendan \& Kutas, 2002, 2003; Schendan \& Lucia, 2010; Schendan \& Maher, 2009). Furthermore, the N3 modulates with perceptual typicality (of a view being unusual vs. canonical, or of an instance or exemplar of a category, e.g., penguin vs. robin), repetition, success of knowledge activation, and visual impoverishment (Ganis \& Schendan, 2011; Maher, 2011; Maher \& Schendan, 2008; Schendan \& Ganis, 2015; Schendan \& Kutas, 2003, 2007a; Schendan \& Lucia, 
2009, 2010; Schendan \& Maher, 2009; Schendan \& Stern, 2007, 2008; Voss et al., 2010). The N3 modulates with the visual similarity to more global, part-like, shape fragments in a view of an instance of a category (Schendan \& Maher, 2009). For example, the N3 shows repetition effects, being larger for new than old repeated objects, that are indistinguishable regardless of whether or not the local lines composing the more global shapes remain the same or differ later on during a memory test compared to the lines composing the object during the learning experience; remarkably, this is true even when no actual lines are shared between learning and test experiences but rather perceptual grouping processes of good continuation and closure between lines enable more global parts and shapes to be perceived (Schendan \& Kutas, 2007a). In contrast, when the overall global shape of a particular exemplar of an object category (e.g., this dog) and its more global parts constructed from the lines instead change between learning and memory test (e.g., the object is seen from a different viewpoint or different exemplars, such as two different dog breeds), then N3 repetition effects become smaller relative to when the global shapes and parts remain the same (Maher, 2011; Maher \& Schendan, in preparation; Schendan \& Kutas, 2003).

\subsubsection{Decision-making}

Several studies timed visual object knowledge activation on open-ended, Go No-Go, 2-choice, and 4-choice category decision tasks with novel and/or repeated objects. Findings show that the $\mathrm{N} 3$ complex is the first ERP to modulate with the success of activating visual object knowledge for the decision (Schendan, 1998; Schendan \& Kutas, 2002, 2003, 2007a; Schendan \& Maher, 2009). Studies that examined single-trial EEG, corresponding ERPs, and EEG-informed fMRI during perceptual decisions about faces and objects provide additional strong evidence for critical decision processes during the N3 complex but not the earlier VPP/N170 (Philiastides et al., 2006; Philiastides \& Sajda, 2006, 2007; Ratcliff et al., 2009; Rousselet et al., 2011). Importantly, this work manipulated both impoverishment (i.e., 6 levels of phase scrambling intermixed randomly) and task (i.e., category vs. color). Activity from 220 to $300 \mathrm{~ms}$ during a frontocentral "D220" (D = difficulty), which MUSI considers to be an N3 component, reflects anterior cingulate, frontal eye field, insula, and dorsal LPFC activity related to task difficulty or task differences in cue diagnosticity (Rousselet et al., 2011). More important, activity from 300 to $450 \mathrm{~ms}$, when the N3 typically peaks, reflects interactive activity between VLPFC and object processing areas for accumulating evidence for the decision; notably, both the D220 and a 'late' component, which are both N3 components, invert polarity posteriorly, as do N3 components at these times. However, potential limitations of this study include the following considerations: analyses focused on faces and so may not generalize to other objects; exemplars were repeated and so effects are conflated with repetition; simple two-choice decisions were assessed (red or green; face or car Philiastides et al., 2006; face identity 1 or 2 Rousselet et al., 2011) and so results may differ with more openended decisions (e.g., confrontational naming); and the category decision required only a superordinate discrimination (i.e., animal or vehicle).

Results from these decision theory studies show that the N3 complex increases while people make category decisions about cars and faces at increasing levels of image impoverishment, and decision-related modulations fit the predictions of decision theory, whereas earlier effects on the VPP/N170 are largely unrelated to performance and reflect only occipitotemporal activity. Work with face images indicates that the N3 but not the earlier VPP/N170 (and EEG at these times) is related to psychometric functions of category decision accuracy and choice probability (Philiastides \& Sajda, 2006). Notably, frontal N3 effects are related to the stimuli and RTs (i.e., more impoverished images yield longer RTs) but not motor execution (J. S. Johnson \& Olshausen, 2005; Philiastides et al., 2006; Philiastides \& Sajda, 2006, 2007; Schendan \& Ganis, 2015). 


\subsubsection{Object Model Selection}

The N3 indexes the most critical process for making a category decision, which is 'object model selection', in which the best match to the percept is activated from among candidate memories (i.e., long-term memory about the visual structure of real objects, e.g., shape, spatial configuration of parts). The N3 complex is the first ERP to differ between perceptually-matched pictures of objects that have been successfully categorized at the entry level relative to those that were unidentified (Doniger et al., 2000; Schendan \& Kutas, 2002), decreasing in amplitude with better categorization and naming success. In one approach to timing of visual knowledge, the N3 complex was shown to be smaller for categorized real objects compared to "pseudo" (unknown, unreal) object versions (Kroll \& Potter, 1984) with matched visual features (Schendan et al., 1998), or other novel, visual structures (Daffner, Mesulam, Scinto, Acar, et al., 2000; Folstein \& Van Petten, 2008; Folstein, Van Petten, \& Rose, 2008; Gruber \& Muller, 2006; Gruber, TrujilloBarreto, Giabbiconi, Valdes-Sosa, \& Muller, 2006; Holcomb \& McPherson, 1994; McPherson \& Holcomb, 1999; Sehatpour et al., 2006). Amplitude of the N3 increases as the categorization task becomes more specific (Schendan et al., 1998). Another approach has been to determine the time when ERPs differ with categorization success, which reveals when the brain starts to use knowledge to categorize the object based on the visual input. Findings indicate that model selection based on visual knowledge starts between 175 and 316 ms, depending upon stimulus, repetition, and task factors (Schendan \& Maher, 2009).

\subsubsection{Implicit memory}

Overall, evidence indicates that modulations of the N3 reflect processes for computing a category decision, learning, and implicit memory with objects (Gruber \& Muller, 2006; Scott et al., 2006; Sehatpour et al., 2006; Soldan, Mangels, \& Cooper, 2006). Regarding memory, the N3 complex shows short- and long-term repetition priming effects on categorization, being smaller for repeated than new objects on tests of implicit memory (Doniger et al., 2001; Ganis \& Schendan, 2008; Gruber, Malinowski, \& Muller, 2004; Gruber \& Muller, 2006; Henson et al., 2004; Schendan \& Kutas, 2003, 2007a; Schendan \& Maher, 2009). The MUSI account is consistent with synchrony models of repetition suppression and priming (Gotts, 2016). N3 and N400 repetition effects resemble those found in fMRI studies of priming, as well as those in animal models (Brozinsky, Yonelinas, Kroll, \& Ranganath, 2005; L. Li, Miller, \& Desimone, 1993), that show neural repetition suppression - that is, less activity for repeated than new objects (Guo, Lawson, \& Jiang, 2007; Henson, 2003); however, the relation between priming and suppression remains to be determined (Desimone, 1996; McMahon \& Olson, 2007), with some favoring a link between repetition enhancement and priming instead (Tartaglia, Mongillo, \& Brunel, 2014). FMRI, N3 and behavioral repetition priming effects are found for categorized real objects but minimally or none for uncategorized objects, indicating priming specifically of object knowledge and meaning, not merely visual features (Schendan \& Maher, 2009; Snodgrass \& Feenan, 1990; Voss, Federmeier, \& Paller, 2012; Voss \& Paller, 2007; Voss et al., 2010). The object knowledge indexed by the N3 complex codes the global shapes of objects and their parts in a specific view, regardless of the constituent local contours composing each shape (Schendan \& Kutas, 2003, 2007a). This is consistent with theories that object categorization is based on similarity to part-like, global shape fragments or features in multiple view representations of each object; note, how visual features are represented remains debated (Biederman, 1987; Borenstein \& Ullman, 2008; Gauthier \& Tarr, 2016; Marr \& Nishihara, 1978; Peissig \& Tarr, 2007; Ullman, 2007; Ullman, Sali, \& VidalNaquet, 2001).

\subsection{N400: Semantic memory and words}


The vast N400 literature has been reviewed extensively elsewhere (Kutas \& Federmeier, 2011) so this paper briefly highlights ideas relevant to MUSI. The centroparietal N400 in response to words and other meaningful stimuli is a negative-going ERP with a centroparietal maximum (with mastoid reference) that peaks around $400 \mathrm{~ms}$ and shows semantic congruency effects between 300 and 500 ms (Kutas, Federmeier, Staab, \& Kluender, 2007; Kutas \& Hillyard, 1980). The N400 has been proposed to reflect semantic memory activation related to processing word-related information in anterior temporal cortex and VLPFC (Kutas \& Federmeier, 2011). Numerous studies indicate the N400 reflects neural processes involved in representing and/or processing semantic knowledge about words (Van Petten \& Luka, 2006). First, N400 amplitude decreases with increasing degree of semantic expectancy for a word in an ongoing sentential context and even in a minimal context, as for semantic priming (i.e., a word pair that shares conceptual information, e.g., doctor-nurse) (Bentin, McCarthy, \& Wood, 1985), indicating the N400 is sensitive to the degree to which the semantic information elicited by a stimulus was previously made active (Kutas \& Federmeier, 2011). Second, N400 size decreases with higher word frequency (usage), at least for words in isolation (Van Petten, 1993); note these frequency effects at least partly reflect semantic factors, since low frequency words likely symbolize concepts invoked less often. Third, N400 amplitude decreases systematically with repetition (Van Petten, Kutas, Kluender, Mitchiner, \& McIsaac, 1991), demonstrating priming. Fourth, fMRI and intracranial ERPs suggest the main generators of the N400 are in VLPFC, especially the BA45 part, and in the ATL and superior temporal gyrus (Friederici, 2002; Halgren et al., 2002; Kutas \& Federmeier, 2011; Marinkovic et al., 2003; Nobre, Allison, \& McCarthy, 1994; Nobre \& McCarthy, 1994, 1995; Patterson et al., 2007; Rossell, Price, \& Nobre, 2003; Van Petten \& Luka, 2006; Willems, Ozyurek, \& Hagoort, 2008).

The MUSI account proposes that the centroparietal N400 indexes later, more abstract, multimodal, associative and complex knowledge processing that may be particularly associated with words, language and analysis of word meaning and semantics. MUSI favors the idea that this more abstract, language-related knowledge is multi, poly, or supra -modal, abstract knowledge (e.g., Chwilla, Virgillito, \& Vissers, 2011; Kutas \& Federmeier, 2000), which is consistent with an embodied framework, in contrast to being an amodal semantic system described in a standard theory of cognition and memory (e.g., Visser et al., 2010). It is important to emphasize that the N400 can be evoked by stimuli other than words, including pictures of objects, faces, scenes and videos. Future work needs to clarify whether this is due to multimodal abstract knowledge or wordrelated knowledge processes that are associated with nonverbal stimuli (e.g., the name associated with an object), or both such abstract and word knowledge.

\subsection{N400 vs. $\mathrm{N} 3$ and amodal vs. embodied cognition}

The MUSI account incorporates evidence for the possibility of different knowledge systems for objects and words. Convergent evidence indicates that the frontal N3 complex and centroparietal N400 differ in their posterior neocortical generators based on where knowledge is stored (Thompson-Schill, 2003). While the N3 reflects activity in VLPFC and posterior objectsensitive areas, the N400 localizes to language-related processing areas in VLPFC and posterior middle and anterior temporal cortex and angular gyrus, especially on the left (E. F. Lau, Phillips, \& Poeppel, 2008; Marinkovic et al., 2003; Van Petten \& Luka, 2006). The N3 and N400 have overlapping functional properties and contributions to state 2 but reflect somewhat distinct neural processes based on their somewhat distinct scalp distributions (Ganis, Kutas, \& Sereno, 1996) and response patterns. It is important to emphasize that pictures have been reported to elicit an N400like ERP, but the scalp distribution tends to be more frontal, like that for the N3 complex, compared 
to when words are the stimuli. This raises the possibility that at least part of the frontal contribution to the N400-like ERP to pictures is instead, or in addition, a component of the frontal N3 complex. The MUSI account allows that the frontal N3 complex may include components that do and do not share functional characteristics with the centroparietal N400. MUSI hypothesizes that more frontal N3 components reflect modal visual object knowledge and spatial structure (Schendan \& Lucia, 2009), while more central N3 components reflect more abstract knowledge, may be multimodal, have a greater semantic processing role akin to the centroparietal N400 (Ganis et al., 1996).

For example, ERPs in response to real objects or famous faces (e.g., Chaby, Jemel, George, Renault, \& Fiori, 2001) show effects of knowledge or semantic congruency with a preceding picture, video or word context, but effects with pictures have a frontocentral maximum, instead of the characteristic centroparietal maximum of the N400 with written words (Bach, Gunter, Knoblich, Prinz, \& Friederici, 2009; Ganis \& Kutas, 2003; Ganis et al., 1996; Sitnikova, Holcomb, Kiyonaga, \& Kuperberg, 2008; Sitnikova, Kuperberg, \& Holcomb, 2003). Critically, semantic congruency effects to pictures versus words in sentences have relatively more frontal versus posterior maxima, respectively, but overlapping distributions such that both pictures and their names show some congruency effects at both frontocentral and parietal sites (Ganis \& Kutas, 2003; Ganis et al., 1996). Such findings raise the possibility that N400-like picture effects reflect both frontal N3 complex and centroparietal N400 effects, rather than purely N400 semantic processing effects. Both pictures and names may activate both visual knowledge associated with the frontal $\mathrm{N} 3$ and more abstract knowledge associated with the centroparietal N400. On the one hand, concrete words that name objects can activate not only their meanings, resulting in N400 modulations, but also associated visual knowledge and other associated knowledge, including embodied information (e.g., sound, action, etc.) through automatic mental simulation mechanisms (Barsalou, 2008; A. Martin, 2007), resulting also in N3 modulations for these words. On the other hand, real objects can activate not only visual knowledge and meanings, resulting in N3 modulations, but also associated names and their word meanings (Humphreys et al., 1999), resulting also in centroparietal N400-like modulations for pictures. Nonetheless, visual memory can be activated independent of abstract and word-related knowledge. For example, visual mental imagery produces frontal N3 effects but little or no centroparietal N400 effects for nonface objects (Schendan \& Ganis, 2012), suggesting N3 and N400 effects can dissociate, and pictorial information can activate visual knowledge and mental simulation without activating the semantic and/or word-related processes associated with the centroparietal N400. Consistent with N3 and N400 dissociation, developmental studies show that, while both 8-9 year old children and adults show centroparietal N400 congruency effects, and adults show frontocentral N300 congruency effects for objects and actions, children show the N300 effects only for objects (notably early at 125-300 ms) (Maguire, Magnon, Ogiela, Egbert, \& Sides, 2013).

Both pictures and concrete words (e.g., names of objects, such as "dog") yield similar ERP maxima that lie anterior to those with abstract words (e.g., "freedom") (Ganis et al., 1996; Holcomb, Kounios, Anderson, \& West, 1999; Kutas \& Federmeier, 2011). Such findings raise the possibility that both pictures and names activate object knowledge associated with the frontal N3 more than do abstract words. Pictures can automatically also activate semantic processing (Boucart, Humphreys, \& Lorenceau, 1995; Pins et al., 2004) and/or word-related processing (e.g., a name) (Humphreys et al., 1999), and, if so, pictures may thus elicit both an N3 component related to visual knowledge and related semantic processing and a centroparietal N400 related to abstract knowledge, semantic and word-related processing. By contrast, abstract words may instead activate semantic processing and/or word-related knowledge associated with the centroparietal 
N400 more than do pictures, videos and names, depending upon the particular stimulus conditions and task demands.

Collectively, this evidence suggests multiple systems for knowledge and semantic memory (Ganis et al., 1996), such as those proposed in embodied (grounded) cognition or dual-coding theories (Barsalou, 2008; Paivio, 1991). Intriguingly, N3 effects largely overlap temporally but can start and peak before N400 effects, placing the N3 in a temporal position to reflect processes supporting mental simulation of object information that constructs the meaning analyses indexed by the N400. Consistent with such mental simulation, mental imagery of shape affects the N3 but not the N400 (Schendan \& Ganis, 2012). Key additional support for this view comes from evidence that modal perceptual processing of the stimulus affects meaning activation (e.g., Federmeier \& Kutas, 2001; Holcomb et al., 1999). This in turn also argues against a sole amodal semantic system, for which the most likely ERP index would be the centroparietal N400. Notably, however, the N400 has been proposed to be multimodal, not amodal (Kutas \& Federmeier, 2011), and so ERPs have not yet provided evidence for a sole amodal semantic system (e.g., Visser et al., 2010).

In summary, the MUSI account allows for the possibility that there may be multiple semantic systems, and neural sources of the N3 complex to pictures include processes related to visual or unimodal knowledge and semantics, whereas those underlying the centroparietal N400 to words includes processes related to more abstract, multimodal, and language-related knowledge and semantic processing. Evidence suggests that N400-like potentials to pictures may be various combinations of $\mathrm{N} 3$ components and the centroparietal N400, not simply the N400 per se. For example, pictures elicit an N3 but may also elicit centroparietal N400-like potentials insofar as word-related, multimodal and/or abstract knowledge or semantic processing is recruited (e.g., name retrieval). On the other hand, N3 components may be elicited by words insofar as visual knowledge or semantics, mental imagery or simulation is activated (e.g., concrete words, sentences emphasizing visual properties).

Nonetheless, it remains also possible that some frontocentral knowledge-related activity in response to nonverbal stimuli like pictures could reflect the abstract knowledge processes associated with the centroparietal N400, but they appear at frontocentral instead of, or in addition to, parietal sites because of somewhat distinct neural generators when the stimulus is a picture instead of a word, such as different regions of VLPFC and the ATL (Gotts, Milleville, Bellgowan, \& Martin, 2011; Simmons \& Martin, 2009). Given the complexity of the neural architecture for knowledge and semantic memory, sorting out these possibilities will be a challenging but important area for future research because the answers will advance understanding of how memory, knowledge and meaning are constructed across brain regions and computational time. The answers will also determine the precise functions of the neurophysiological activity indexed by the N3 and N400 and the role of these functions in cognition, decisions, memory, meaning and consciousness. Future work should also explore the possibility that the N3 complex includes modal higher-order, knowledge-related semantic processes, and the N400-like response to pictures reflects a combination of both $\mathrm{N} 3$ components and centroparietal N400 effects of multimodal abstract knowledge and semantic processing, with the relative contributions varying with the experimental parameters.

\subsection{Brain Basis of Top-Down Perceptual Hypothesis Testing (PHT) for Object Constancy}

The MUSI account incorporates PHT to explain the brain dynamics for flexibly activating memory for decisions about novel shapes and facilitating the perceptual constancy of object cognition (Schendan \& Ganis, 2015). The MUSI account proposes that PHT processes occur first during state 2 . Key evidence for this is that category decisions take different amounts of time, 
depending upon the stimulus and task demands. ERP negativities after $200 \mathrm{~ms}$ reflect this, but not earlier ERPs, including the VPP/N170 index of initial bottom-up activity in occipitotemporal cortex in state 1. Furthermore, bottom-up computational models perform worse than people when the visual system is given or requires more time to accumulate evidence before the decision, as for open-ended categorization (Serre, Wolf, et al., 2007) and highly impoverished stimuli (Ganis et al., 2007; Palmer et al., 1981; Schendan \& Kutas, 2003; Schendan \& Stern, 2008; Tarr, Williams, Hayward, \& Gauthier, 1998). In these cases, people are still accurate, but slower (Potter \& Faulconer, 1975): Accuracy costs time. These limitations led some to suggest that the bottom-up pathway provides the initial input and object hypothesis to test using subsequent top-down processes of PHT (Serre, Wolf, et al., 2007). Further, computational modeling increasingly supports a critical role for top-down influences about category knowledge on lower-level cortex (e.g., Yang et al., 2019).

PHT theories propose that top-down inputs facilitate object constancy, but each variant of this type of theory proposes different neural mechanisms. PHT top-down input could originate from within the ventral visual hierarchy itself (i.e., from higher to lower order areas) and/or from other areas, especially parietal and/or lateral prefrontal cortex; see for review Schendan and Ganis (2015). MUSI incorporates a hybrid PHT theory that combines two frontoparietal variants for which convergent evidence indicates PHT processes occur for the first time during state 2, as well as related computational accounts of neural mechanisms; in contrast, most other PHT theories focus on earlier, bottom-up and/or automatic feedback processes during state 1. For this hybrid theory, the main framework comes from model verification theory (Kosslyn et al., 1994; Lowe, 1985, 2000), and multiple-views-plus-transformation (MVPT) theory provides details about memory representations and spatial processing (Bülthoff et al., 1995; Tarr \& Pinker, 1989). Further, neural mechanisms are based on computational models involving hierarchical Bayesian inference (T. S. Lee \& Mumford, 2003), adaptive resonance theory (ART) (Fazl et al., 2009; Grossberg, 1999), or predictive coding (Friston \& Kiebel, 2009). Convergent evidence supports verification and MVPT variants that are unique in implicating frontoparietal contributions after the initial bottom-up activation of the ventral stream, with PHT processes starting around $250 \mathrm{~ms}$ in state 2 (Schendan \& Ganis, 2015). These frontoparietal computations are the most important modulators of posterior neocortex in order to achieve object constancy(Ganis et al., 2007; Schendan \& Ganis, 2015; Schendan \& Lucia, 2012; Schendan, Lucia, \& Bridges, submitted; Schendan \& Stern, 2008).

According to a hybrid MVPT-verification PHT theory, top-down processes in prefrontal and posterior parietal cortex drive iterative PHT processes to determine the object memory in occipitotemporal neocortex that best explains the percept. When sensory conditions are optimal or for less impoverished images, the percept can be matched to the correct memory (or 'object model') and a decision can start to be achieved (state 2) largely, if not entirely, based on information computed by the end of the initial bottom-up pass through the posterior visual processing hierarchy (state 1) with minimal or no additional processing (e.g., Rousselet, Thorpe, \& Fabre-Thorpe, 2004). MVPT proposes that multiple views of an object are stored in memory. When an object is observed from a viewing angle that is known, familiar, or canonical (i.e., yields the best performance (Blanz, Tarr, \& Bulthoff, 1999; Palmer et al., 1981), the view memories activated during the initial bottom-up pass through the ventral visual hierarchy are sufficient to make a decision (Kosslyn et al., 1994; Tarr \& Pinker, 1989).

By contrast, for more impoverished images, the bottom-up pass may find only an initial weak match to visual knowledge about objects (i.e., a weakly activated candidate "visual model", 
hypothesis, or predicted category). Consequently, two top-down PHT processes (Ganis et al., 2007) are recruited that involve cognitive control, working memory, and selective attention functions in frontoparietal cortex (Courtney, 2004; Dolan et al., 1997; Kastner \& Pinsk, 2004; Postle, 2006) that interact with and modulate object processing in posterior object-sensitive cortex (Ganis et al., 2007). These two processes cycle iteratively until the task is done (e.g., a decision is made), or fails. Each cycle ends with a "reset" signal to inhibit the rejected hypothesis and allow the next cycle to start (Fazl et al., 2009). (1) The first PHT process is hypothesis 'prediction', which predicts the perceptual hypothesis, object model or category that corresponds to the sensory input. Prediction uses the perceptual input and contextual (linguistic or scene) information to find the best match between the perceived object and memory (Ganis et al., 2007; Schendan, submitted; Schendan \& Stern, 2008) and may use predictive coding computations (Friston \& Kiebel, 2009). (2) The second PHT process is hypothesis 'testing', which evaluates the similarity of the spatial configuration of features between the perceived object and the predicted model in memory (Ganis et al., 2007; Schendan \& Stern, 2008). The spatial configuration of parts is crucial for object categorization (Behrmann \& Williams, 2007; Biederman, 1987; Cave \& Kosslyn, 1993). Spatial processes implicated in mental rotation, as posited in MVPT, have been proposed to have a role in testing (Aso et al., 2007; Schendan \& Stern, 2007, 2008) According to MVPT, for viewing angles that are unknown, unfamiliar, or unusual, additional spatial processes are recruited to transform and align the perceived image with the view representations in memory to try to achieve a successful match (Bülthoff et al., 1995). Spatial transformation can include frontoparietal processes required for the cardinal spatial task of mental rotation, wherein people decide whether two objects rotated relative to each other are the same or different (Tarr \& Pinker, 1989).

Key evidence for a hybrid MVPT-verification PHT theory includes the following. FMRI and positron emission tomography studies have manipulated image impoverishment (e.g., added noise, deleted features, fog). PHT theories predict the opposite of bottom-up accounts for more relative to less impoverished images: PHT processes should show greater neural activity (not less, as for bottom-up processes) in object-sensitive occipitotemporal cortex, prefrontal areas for cognitive control (Courtney, 2004; Postle, 2006) and posterior parietal areas for spatial transformation and object mental rotation (Schendan \& Stern, 2007). Indeed, findings implicate top-down processes from parietal and LPFC areas (Baird, Colvin, Vanhorn, Inati, \& Gazzaniga, 2005; Ganis et al., 2007; Kosslyn et al., 1994; Schendan \& Stern, 2008; Sugio et al., 1999). For example, categorizing and recognizing more relative to less impoversished images of objects (fragmented line drawings, unusual views) activates a network of LPFC, occipitoparietal, and occipitotemporal regions, and this impoverishment effect is greater for real than "pseudo-objects" (i.e., unknown or unreal objects), demonstrating the modulation specifically relates to object knowledge (Ganis et al., 2007; Kosslyn et al., 1994; Schendan \& Stern, 2008; Sugio et al., 1999). Further, one fMRI study identified task general and categorization-specific processes (Schendan \& Stern, 2008).

However, these neuroimaging methods have high spatial but poor time resolution. Using ERPs, which have high time precision, with the same methods as the fMRI studies of object constancy captured the timing of impoverishment effects and task effects (Schendan \& Ganis, 2015; Schendan et al., submitted). Regarding timing, this hybrid frontoparietal PHT theory predicts that top-down processes affect occipitotemporal processing after the initial bottom-up pass in state 1 when top-down frontoparietal interactions implement PHT. This predicts impoverishment effects in ERPs (more $v s$. less impoverished) after 200 to $250 \mathrm{~ms}$ when state 2, top-down frontoparietal modulations and spatial cognition starts or after $\sim 500 \mathrm{~ms}$ when effortful 
spatial processes that support strategic mental rotation start (Schendan \& Lucia, 2009). Consistent with this, the N3 complex is the first ERP to show impoverishment effects: Negativity is greater for more than less impoverished images of real objects (Schendan \& Kutas, 2003; Schendan \& Maher, 2009). Thus top-down PHT processes facilitate the visual constancy of object cognition for the first time in state 2 during the N3. Consistent with the N3 as an index of top-down processes that activate visual memory, evidence indicates that mental imagery and prior semantic context modulate the frontal N3 (e.g., Ganis \& Kutas, 2003; Ganis et al., 1996; Schendan \& Ganis, 2012; Schendan \& Lucia, 2009; Sitnikova et al., 2008).

Human fMRI and ERP evidence suggests that the two PHT processes operate in state 2 between 200 and $500 \mathrm{~ms}$ and suggest where, when and how PHT processes happen. For visual object constancy, neuroimaging implicates VLPFC (Ganis et al., 2007; Schendan \& Stern, 2008), which has the most extensive connections with the ventral visual stream (Petrides \& Pandya, 2001), a general role in memory retrieval and selection (e.g., Badre, 2008), and a special role in category decisions (Freedman, Riesenhuber, Poggio, \& Miller, 2003; Jiang et al., 2007). The MUSI account hypothesizes the following. (1) Prediction involves mid-VLPFC (BA 47/12; BA 45) and lateral occipital sulcus (LOS) and right fusiform neocortex along the ventral pathway, and this process is indexed by a frontocentral N390 component of the N3 complex (Ganis \& Schendan, 2008, 2011; Schendan \& Kutas, 2003; Schendan \& Stern, 2008). This neurophysiological activity is task-dependent (e.g., involved in categorization more than episodic recognition, though it has a role in both) (Ganis et al., 2007; Jiang et al., 2007; Kosslyn et al., 1994; Raposo, Han, \& Dobbins, 2008; Schendan \& Stern, 2008). The BA 47/12 part of VLPFC may predict by flexibly selecting, retrieving, disambiguating and classifying, and actively maintaining visual object information and memory in the LOS until a decision is made (Cisek, 2007; Gotts et al., 2011; Heekeren, Marrett, \& Ungerleider, 2008; Kostopoulos, Albanese, \& Petrides, 2007; Kostopoulos \& Petrides, 2003, 2008a, 2008b; Postle, 2006; Wig, Grafton, Demos, \& Kelley, 2005). LOS may predict based on more local features (shape fragments). BA 47/12 in the left hemisphere may have a greater role in linguistic, abstract, local level, or rule knowledge and that in the right hemisphere may have a greater role in nonverbal and global shape information and memory(Badre \& Wagner, 2007; Bookheimer, 2002; Bunge, 2004; Dobbins \& Wagner, 2005; Ivry \& Robertson, 1998; Kan \& Thompson-Schill, 2004; Oztekin, McElree, Staresina, \& Davachi, 2009; Schendan \& Kutas, 2007a; Schendan \& Stern, 2008). These object model prediction processes are indexed by a frontocentral component of the N3. In addition, prediction may involve abstract object knowledge, including linguistic knowledge, indexed by the centroparietal N400. This more abstract contribution may reflect a role of BA 45 to predict more abstractly by elaborating retrieved memory selected from posterior memory storage areas, especially the left ATL for linguistic knowledge (e.g., a verbal label) and the right ATL for nonlinguistic knowledge (e.g., perceptual features of an object), extending into surrounding occipitotemporal and parahippocampal regions (Badre \& Wagner, 2007; Gorno-Tempini et al., 2004; Marinkovic et al., 2003; A. Martin, 2007; C. B. Martin, Cowell, Gribble, Wright, \& Kohler, 2016; Raposo et al., 2008; Thompson-Schill, 2003; Van Petten \& Luka, 2006). Accordingly, BA 47/12 connects mainly with occipitotemporal cortex, implicating it in shape prediction, and BA 45 connects mainly with auditory processing areas, implicating it in linguistic and more abstract knowledge prediction (Petrides \& Pandya, 2001). Notably, prefrontal cortex, especially VLPFC, may contribute not only to memory retrieval but also to representing conceptual knowledge in a flexible, context-specific way (Gotts et al., 2011).

The model testing process recruits a network involving ventral premotor parts of a caudal 
VLPFC region (PMv; BA 44/6), an occipitoparietal region, and the inferotemporal sulcus (ITS), which are task-independent, showing impoverishment effects on both categorization and episodic recognition (Schendan \& Stern, 2008). This is indexed by a frontopolar component of the N3 (a.k.a. N350). The model testing process supports diverse cognitive tasks (e.g., categorization, recognition, mental rotation) (Schendan \& Lucia, 2009, 2010; Schendan \& Stern, 2008). Accordingly, PMv connects strongly with the intraparietal region (Grezes, Armony, Rowe, \& Passingham, 2003), consistent with model testing based on spatial analysis of objects. The occipitoparietal region encompasses 2 retinotopic, object-sensitive areas: the ventrocaudal intraparietal sulcus (vcIPS, including area V7) and the transverse occipital sulcus (TOS, including area V3A) (Carpenter, Just, Keller, Eddy, \& Thulborn, 1999; Denys et al., 2004; Grill-Spector, Kourtzi, \& Kanwisher, 2001; Grill-Spector, Kushnir, Hendler, \& Malach, 2000; I. M. Harris et al., 2000; Podzebenko, Egan, \& Watson, 2002; Sawamura, Georgieva, Vogels, Vanduffel, \& Orban, 2005; Schendan \& Stern, 2007; Swisher, Halko, Merabet, McMains, \& Somers, 2007); note, more anterior intraparietal areas for spatial analysis and object-based properties (Shomstein \& Behrmann, 2006) have less of a role in model verification and overlap saccade areas (Schendan \& Stern, 2007). The vcIPS is more size- and view-specific and less sensitive to repetition than occipitotemporal cortex, (Grill-Spector et al., 1999; James, Humphrey, Gati, Menon, \& Goodale, 2002; Sawamura et al., 2005; Schendan \& Stern, 2008; Sugio et al., 1999; Vanrie, Beatse, Wagemans, Sunaert, \& Van Hecke, 2002), though TOS shows position-invariant adaptation (MacEvoy \& Epstein, 2007). The occipitoparietal region has been implicated in mental rotation, visual working memory (or visual short-term memory) for automatically tracking the relative spatial location of features within an object, object grouping and object-based representation ("object files"), individuating objects, and binding features into an object token representation (Epstein, Harris, Stanley, \& Kanwisher, 1999; Epstein \& Kanwisher, 1998; Friedman-Hill, Robertson, \& Treisman, 1995; Kim \& Robertson, 2001; Oztekin et al., 2009; Robertson, 2003; Schendan \& Stern, 2007, 2008; Turriziani, Carlesimo, Perri, Tomaiuolo, \& Caltagirone, 2003; Wendelken, Bunge, \& Carter, 2008; Xu, 2008; Xu \& Chun, 2006, 2007). The ITS shows impoverishment and mental rotation effects consistent with a role in model testing and may contribute information and memory about the spatial configuration of features (Schendan \& Stern, 2007, 2008). Accordingly, the vcIPS region modulates neuronal activity in more ventral cortex, such as ITS, in ways that fundamentally affect the object representations (Aviezer et al., 2007; Friedman-Hill, Robertson, Desimone, \& Ungerleider, 2003; Ganis et al., 2007; McCloskey, 2004), and monkey ventral parietal cortex projects to inferotemporal area TE, which exhibits both shape and spatial configuration properties (Messinger, Squire, Zola, \& Albright, 2005; Zhong \& Rockland, 2003). Notably, both prediction and testing regions are also recruited for the top-down, spatial mental imagery task of object mental rotation (Aso et al., 2007; Schendan \& Stern, 2007, 2008), implicating PHT processes in mental rotation, and vice versa.

Prediction-testing cycles iterate in state 2 during the N3 complex, but PHT can start with testing because the initial bottom-up activation can compute the first prediction (Serre, Wolf, et al., 2007). For more impoverished objects, since the first prediction is likely poor, the first test determines that this predicted model only weakly matches memory and so recruits the first model prediction attempt using PHT processes in state 2. This sequence (i.e., bottom-up yields the first predictionfirst test - second prediction then iterative prediction-testing) is consistent with the observation that frontopolar N3 effects (testing) start and peak earlier than frontocentral N3 effects (prediction). However, if the bottom-up pass fails to yield a prediction, then prediction should precede testing, leading to variation in timing of $\mathrm{N} 3$ components depending on the situation. 
Evidence indicates that knowledge underlying the N3 has a greater role in object constancy than the knowledge underlying the N400, and the semantic activations indexed by the N400 have less of a role in category decisions than perceptual knowledge associated with the N3 complex. FMRI and N3 viewpoint effects indicate greater processing of more (unusual) than less (canonical) views, consistent with frontoparietal PHT accounts of object constancy (Ganis et al., 2007; Schendan \& Ganis, 2015; Schendan \& Kutas, 2003; Schendan et al., submitted; Schendan \& Stern, 2008). The N400 shows small viewpoint effects, being larger for unusual than canonical views during categorization of real objects from 390 to $500 \mathrm{~ms}$ (Schendan \& Kutas, 2003). This suggests that word-related semantic processing underlying the N400 has a role in prediction for PHT or N400 semantic processing includes visual knowledge, perhaps as part of its multimodality. However, such effects are not reliable and vary by task, being undetectable on an episodic recognition task (Schendan \& Lucia, 2012; Schendan et al., submitted). Regardless, this suggests that, relative to knowledge processes underlying the N3 complex, semantic processes underlying the N400 have a minimal role in episodic recognition and less of a role in categorization. This is consistent with evidence that mediotemporal damage impairs episodic memory and a late positive complex associated with episodic memory in state M, but the N400 remains largely unaffected, being instead abnormal in people with damage to neocortical association areas (J. R. Taylor \& Olichney, 2007).

\subsection{N3(00) Components \& Brain Basis Support Functional Significance of State 2}

Next, studies of the N3 complex are reviewed that further explain the functional significance and properties of state 2, dissociations between states and between frontopolar and frontocentral $\mathrm{N} 3$ components. Convergent evidence suggests that the N3 is a complex of functionally-distinct components with different cortical sources (e.g., Schendan \& Maher, 2009; Sehatpour et al., 2006).

\subsubsection{Target N250: Ultra-Rapid Categorization starting by 170 ms (Decisions Based on Initial} Bottom-Up Pass)

The 'target N250' marks the transition between state 1 and 2 when processing switches from predominantly bottom-up to predominantly recurrent, feedback, top-down and interactive processes that operate according to the task goal. One important source of evidence for this transition comes from ERP studies that aimed to determine the timing of categorization based on the initial bottom-up pass through the ventral visual stream using 'ultra-rapid categorization' tasks, on which performance is fastest ( $420 \mathrm{~ms}$ ), compared to other tasks. These studies primarily used tasks involving two-choice decisions about the global "target" category of an object (e.g., animal or not). Subjects are instructed to respond to the target category in complex natural scenes, then the scenes are flashed briefly (20-40 ms) followed by a visual mask (Delorme, Richard, \& FabreThorpe, 2000). Subjects report whether the target was present.

The processing difference between targets and non-targets can be detected in the ERPs with an onset at the earliest of between 154 and $170 \mathrm{~ms}$, modulating a 'target N250', a negativegoing ERP peaking around $250 \mathrm{~ms}$ with a maximum at frontocentral locations. The N250 is less negative to scenes where the target category is successfully detected, as indicated by a "Go" RT, relative to when the target is absent, indicated by no response ("NoGo") (Bacon-Mace et al., 2005; Fabre-Thorpe, Delorme, Marlot, \& Thorpe, 2001; Thorpe et al., 1996; R. VanRullen \& S. J. Thorpe, 2001). The target effect has been shown not to be attributable merely to feature, motor, category level, and masking factors, but the target effects depend on cognitive and task demands (Bacon-Mace et al., 2005; Delorme et al., 2000; Fabre-Thorpe et al., 2001; J. S. Johnson \& Olshausen, 2003, 2005; R. VanRullen \& S. J. Thorpe, 2001): The N3 is larger with (i) two-choice than go-no go tasks, (ii) RTs that are faster than slower, (iii) multiple than single category tasks, 
and (iv) categorization at the basic level (e.g., dog) compared to a more general, superordinate level (e.g., animal). The brain source of the N250 target effect, which was measured between 190 and $215 \mathrm{~ms}$, has been localized to occipitotemporal cortex (Delorme, Rousselet, Mace, \& FabreThorpe, 2004; Fize, Fabre-Thorpe, Richard, Doyon, \& Thorpe, 2005). This has been widely accepted as strong evidence that decisions about real objects in complex scenes can be accomplished primarily using powerful discriminative information computed in the initial feedforward sweep along the visual hierarchy from lower-level occipital to higher-level temporal cortex in humans by the 154 to $170 \mathrm{~ms}$ onset of the target N250 effect (Fabre-Thorpe et al., 2001; Riesenhuber \& Poggio, 2002, 2004; Rolls, Tovee, \& Panzeri, 1999; Schoenfeld et al., 2003; Thorpe et al., 1996; VanRullen \& Koch, 2003; R. VanRullen \& S. Thorpe, 2001). Critically, this onset time is 30 to $50 \mathrm{~ms}$ later than the $120 \mathrm{~ms}$ start time of categorical perception during the VPP/N170 in state 1, which shows no evidence of a target effect. Thus, the target N250 effect is too late to capture the initial bottom-up activation of the human ventral visual pathway, which instead is captured by the earlier VPP/N170 effects of categorical perception starting at $120 \mathrm{~ms}$. Notably, studies have implicated interactions among top-down prefrontal feedback and bottom-up processes in fusiform cortex as early as $180 \mathrm{~ms}$ in visual object cognition (Bar et al., 2006), but these are small, specialized effects. Moreover, $180 \mathrm{~ms}$ and the 154 to $170 \mathrm{~ms}$ onset of target N250 responses are still long after the $120 \mathrm{~ms}$ onset of VPP/N170 effects of categorical perception.

The location, time course, and functional characteristics of the target N250 indicate it is a component of the frontal N3 complex, possibly the earliest part of the frontocentral N3 component, and, according to the MUSI account, the onset of the target N250 during ultra-rapid categorization tasks pinpoints the end of the initial bottom-up pass through the ventral stream (state 1) and the start of decision and other higher cognitive processes (state 2). Convergent evidence indicates that state 1, indexed by early ERPs (e.g., VPP/N170) between $120 \mathrm{~ms}$ until around 170 to $250 \mathrm{~ms}$, involves predominantly bottom-up computations, and, starting around 154 to $170 \mathrm{~ms}$, in state 2, processing becomes increasingly dominated by interactive recurrent and feedback processes (e.g., task goal information feeding back from PFC to posterior cortex). For example, top-down prefrontal influences modulate the target N250 (Delorme et al., 2004), and N250 effects reflect 'post-sensory decision processes' (J. S. Johnson \& Olshausen, 2003, 2005).

\subsubsection{Open-Ended Category Decisions Involve Recurrent and Feedback Processes}

When the decision is open-ended or sensory input is impoverished or ambiguous (see Sections 1.5 and 4.1), then additional processes are necessary. People are much faster at categorizing objects when the target class is pre-specified (Potter \& Faulconer, 1975), as on ultra rapid tasks, than when they must discern the relevant category themselves. Ultra-rapid target detection tasks minimize critical, neural computations that are normally used on "open-ended" decision tasks, such as basic or entry level categorization (e.g., during confrontational naming or when meaning or semantic memory is task-relevant). On open-ended tasks, the visual system has to discriminate one specific class of visual objects from all others with little or no advance information to cue the category (Enns, 2004). Fast bottom-up, hierarchical processing in occipitotemporal cortex is necessary for perceiving the object (Ganis \& Schendan, 2008; Horovitz et al., 2004), but this may not be sufficient for a decision, nor for higher cognition or awareness, motivating the MUSI proposal that these happen in state 2.

\subsubsection{Frontopolar vs. Frontocentral N3s}

In the MUSI account, frontopolar N3s reflect decision processes involving spatial information about objects and hypothesis testing, whereas frontocentral N3s reflect decision or significancedetermining processes involving nonspatial, salient feature, semantic or associative knowledge, 
and hypothesis prediction.

3.5.3.1 Frontopolar N3: Visual spatial object structure

Effects of novel visuospatial structure (e.g., real vs. pseudo objects) are largest on an N3 component from 200-500 ms maximal at frontopolar locations. The frontopolar N3 component decreases as the percept is rated as a better match to object knowledge (Schendan \& Maher, 2009). Notably, this relationship is consistent with an embodied knowledge account of categorization in which perceptual similarity has a crucial role (Goldstone, 1994; Goldstone \& Barsalou, 1998; Kruschke, 2008; Shepard, 1987), and evidence that shape similarity drives occipitotemporal activity (Freedman, Riesenhuber, Poggio, \& Miller, 2001, 2002; Freedman et al., 2003; Jiang et al., 2007; Kriegeskorte, Mur, Ruff, et al., 2008; L. Li et al., 1993; Op de Beeck, Wagemans, et al., 2008; Rainer \& Miller, 2000; Sigala, 2004; Sigala, Gabbiani, \& Logothetis, 2002; Sigala \& Logothetis, 2002). The N3 has a frontopolar maximum for effects that a hybrid MVPT-verification PHT theory hypothesizes involves more spatial processing in the testing system: (a) impoverishment effects during categorization, (b) repetition and category decision effects with more impoverished objects (Schendan et al., 1998; Schendan \& Kutas, 2002, 2003, 2007a; Schendan \& Maher, 2009), and (c) stimuli that are relatively atypical or structurally novel for a category (Folstein \& Van Petten, 2004, 2008; Folstein et al., 2008; Gratton et al., 2009; Schendan \& Kutas, 2003; Schendan \& Lucia, 2009; Schendan \& Stern, 2007, 2008). Further, an ERP version of an fMRI study of object mental rotation dissociated N3 components: Frontopolar N3s between 200 and $700 \mathrm{~ms}$ show robust linear rotation effects like those in occipitoparietal parts of the testing system for PHT, whereas frontocentral N3s do not (Schendan \& Lucia, 2009). Overall, evidence also suggests the frontopolar N3 indexes automatic mental simulation to embody cognition in the visuospatial configuration of an object during state 2 .

3.5.3.2 Frontocentral N3: Visual feature, object and semantic information, knowledge and memory

In contrast, the N3 has a frontocentral maximum (e.g., N390) between 200 and 500 ms for semantic congruency effects with visual objects for which negativity becomes less when the category has been predicted by a preceding sentence or picture context (Barrett \& Rugg, 1990; Ganis \& Kutas, 2003; Ganis et al., 1996; McPherson \& Holcomb, 1999; Schendan \& Maher, 2009; Sitnikova et al., 2008; Sitnikova et al., 2003). When specified using a scene context, semantic congruency effects for objects show a frontocentral N3 but no such frontopolar effect (Ganis \& Kutas, 2003), dissociating these components. Frontocentral N3 effects of category decisions and image impoverishment are task-dependent, reflect interactions between the right VLPFC (BA 47/12) and occipitotemporal cortex, especially the LOS (Schendan \& Lucia, 2012; Schendan et al., submitted; Schendan \& Stern, 2008). MUSI thus proposes the frontocentral N3 indexes the network for a PHT prediction process. Consistent with this, the prediction system for PHT has been implicated in deciding the category of an object using conceptual knowledge (Jiang et al., 2007; A. Martin, 2007; Schacter, Wig, \& Stevens, 2007). Thus category decisions involve the PHT prediction network; note, how to link neural data to decision theory remains uncertain, with some suggesting that PMv and vcIPS (indexed by the frontopolar N3) are more important than VLPFC (Gold \& Shadlen, 2007; Heekeren et al., 2008; Philiastides \& Sajda, 2007; Ploran et al., 2007; Ratcliff, 1978; Wheeler, Petersen, Nelson, Ploran, \& Velanova, 2008).

Some of the same factors that affect the centroparietal N400 to word stimuli also modulate frontal N400-like ERPs to pictures of non-linguistic visual objects, which herein are suggested to include frontocentral N3 components (see also section 3.3). First, the N400 amplitude to pictures of living and nonliving objects decreases when preceded by semantically-related words and 
sentences (Federmeier \& Kutas, 2001; Ganis et al., 1996; Kiefer, 2001). Second, the frontocentral N3 and centroparietal N400 decrease when the identical famous face is vividly imagined (cued by its name) before the picture is presented, but nonface objects show only N3 complex effects (Schendan \& Ganis, 2012), indicating stimulus-dependence of effects. Third, frontal N400-like ERPs are also modulated by non-linguistic visual contexts: single objects (Barrett \& Rugg, 1989, 1990; Holcomb \& McPherson, 1994; McPherson \& Holcomb, 1999), scenes (Ganis \& Kutas, 2003), and video clips (Sitnikova et al., 2008; Sitnikova et al., 2003). In line with these priming effects, the N400-like object congruency effect is accompanied by behavioral semantic priming, that is, faster RTs for objects categorized following a semantically predictive than nonpredictive scene context (Ganis \& Kutas, 2003).

Compared to the centroparietal N400 to words, picture effects have a frontocentral scalp distribution and overlapping time course yet can start and peak earlier, resulting in labels like N300 (McPherson \& Holcomb, 1999) and frontocentral N390 (Ganis \& Kutas, 2003) (i.e., components of the N3 complex). For example, semantic congruency effects at frontocentral head locations with pictures can start and peak earlier than those at centroparietal N400 locations with words (Ganis \& Kutas, 2003; Ganis et al., 1996); note, with pictures of objects, the centroparietal N400 can occur or peak later, for example, appearing as an "N450" (e.g., Barrett \& Rugg, 1990; Sitnikova, West, Kuperberg, \& Holcomb, 2006). In particular, centroparietal N400 linguistic effects reliably start by 250 to $300 \mathrm{~ms}$ and semantic effects start by $300 \mathrm{~ms}$ (Laszlo \& Federmeier, 2014). Most important, when congruency effects in sentences are compared directly for perceptually-matched line drawings of objects and words (Ganis et al., 1996), both peak around the same time (410 vs. $416 \mathrm{~ms}$, respectively) when presented in separate blocks. However, when intermixed, N400-like ERPs peak earlier for pictures than words (397 vs. $414 \mathrm{~ms}$, respectively, with the largest picture advantage $(30 \mathrm{~ms})$ at parietal sites. Moreover, only for pictures, the congruency effect starts around $150 \mathrm{~ms}$ and is significant between 150 and $275 \mathrm{~ms}$ (earlier than with words) at frontocentral N3 sites but not at centroparietal N400 sites (Ganis et al., 1996). Thus semantic congruency effects for pictures relative to words can start up to $125 \mathrm{~ms}$ earlier due to early effects only for pictures at frontal N3 sites and can peak earlier at both frontal N3 and centroparietal N400 sites. Further, for pictures relative to words, visual knowledge and abstract semantic processes can not only start earlier but also have a faster time course of processing. This places the visual knowledge underlying the frontal $\mathrm{N} 3$ complex in a temporal position (potentially earlier yet overlapping) to embody, mentally simulate and otherwise contribute to constructing meaning using more advanced, multimodal and abstract knowledge and semantic processes during the centroparietal N400. Overall, evidence suggests functional similarity but anatomical differences between word vs. object knowledge (and associated semantic processes) underlying the linguistic centroparietal N400 vs. frontocentral N3, respectively.

Semantic priming links these studies on word and object knowledge to those on ultra-rapid categorization (see also section on 'Target N250: Ultra-Rapid Categorization by $170 \mathrm{~ms}$ '). In standard semantic priming paradigms, exposure to an item, the "prime", a brief time before (i.e., $<$ about $1 \mathrm{~s}$ ) improves performance with the next item, the "target", which it resembles in meaning but not in sensory form (e.g., the word "dog" and then a picture of a dog). From a memory standpoint, both semantic congruency and ultra-rapid categorization include key elements of semantic priming paradigms: The "prime", which is presented just beforehand, affects subsequent processing of the semantically-related word or picture "target"; for semantic congruency paradigms, the prime is a linguistic or picture context, and the target stimulus is a word or object; for ultra-rapid categorization, the prime is the name of a category (e.g., "animal"), and the target 
stimulus is a complex scene of objects, which includes the target (e.g., an animal) or not. Indeed, some ultra-rapid categorization studies used a "name verification" task involving a semantic priming paradigm: A name (e.g., "dog") primes the subsequent target object in the scene context, and subjects verify the scene as containing the named target or not (J. S. Johnson \& Olshausen, 2003, 2005). ERP negativity decreases to scenes with the target (i.e., the primed object) relative to those without it, between $\sim 150$ and $500 \mathrm{~ms}$. This 'target N250' has the waveform, timing, and scalp distribution of the frontocentral N3 component (J. S. Johnson \& Olshausen, 2003), contributing to the earliest part of this N3. Studies that manipulated the original 'ultra-rapid' task and the name verification variant demonstrated that these effects cannot be attributed merely to feature, motor, category level, and masking confounds, but some factors in the 'ultra-rapid' paradigm minimized the ERP effect, as follows. (a) Backward masking likely minimized top-down processing (Di Lollo, Enns, \& Rensink, 2000; Enns, 2004; Enns \& Di Lollo, 2000; Enns, Lleras, \& Di Lollo, 2006). (b) Categorization at the superordinate level (animal) results in a smaller N3 and smaller target effects than at the basic level (dog), indicating that better prediction reduces the N3. (c) The easy Go-No Go task (in the seminal ultra-rapid experiments) results in an N3 that is earlier and smaller, and RTs that are faster, relative to the 2-choice task used for name verification (Gomez, Ratcliff, \& Perea, 2007). (d) Target N250 effects with a single category are much smaller than with many categories; (Ganis \& Schendan, 2008; Rossion et al., 2002; Schendan et al., 1998; Scott et al., 2006; J. W. Tanaka \& Curran, 2001). Results also show the following. (e) Image impoverishment (e.g., part deletion) decreases and delays the target N250 effect, which is smaller and later when RTs are slower, consistent with findings that the frontocentral N3 indexes PHT processes of prediction for object constancy (Ganis et al., 2007; Schendan \& Kutas, 2003; Schendan \& Stern, 2008). (f) The centroparietal N400 to words typically also shows these effects, as expected if the word prime activates both name (N400) and object knowledge (N3). Altogether, ERP studies using semantic priming paradigms demonstrate that prediction of object knowledge reduces the frontocentral N3 index of a VLPFC-posterior object-sensitivenetwork, and improves object memory activation.

\subsubsection{PHT: Frontopolar N3 indexes testing and frontocentral N3 indexes prediction}

The MUSI account proposes that state 2 also contributes to PHT, but the frontopolar N3 indexes testing and frontocentral N3 indexes prediction. Accordingly, the N3 complex is the first ERP to show impoverishment effects: Negativity is greater for more than less impoverished images of real objects (e.g., unusual than canonical views, and more than less fragmented line drawings, respectively) between 200 and 500 ms (Schendan \& Ganis, 2015; Schendan \& Kutas, 2002, 2003; Schendan \& Maher, 2009); note, the centroparietal N400 also shows an effect in some studies (Schendan \& Ganis, 2015; Schendan \& Kutas, 2003). Frontopolar and frontocentral N3s show the same modulations with key exceptions that implicate the frontopolar N3 as an index of PHT testing. (a) The frontopolar N3 can show less or no effects of object congruency and semantic priming, as opposed to the larger and consistent effects on the frontocentral N3 (Ganis \& Kutas, 2003). (b) The frontopolar N3, but not the frontocentral N3, shows linear modulations with categorization success (Schendan \& Maher, 2009). (c) The frontopolar N3, but not the frontocentral N3, from 200 to 400 ms shows sustained linear effects of object mental rotation like those found in the occipitoparietal region of the testing system using fMRI (Cohen et al., 1996; Podzebenko et al., 2002; Podzebenko, Egan, \& Watson, 2005; Schendan \& Lucia, 2009; Schendan \& Stern, 2007). (d) The frontopolar N3 to a picture (of a real object or famous face) decreases when preceded by vividly imagining that same picture (cued by its name) relative to a different picture (Schendan \& Ganis, 2012). This suggests that the PHT testing network is a brain source of 
the frontopolar N3.

Prediction is indexed by the frontocentral N3. Accordingly, evidence indicates that prediction of visual object knowledge improves categorization and reduces the frontocentral N3, which localizes to VLPFC and LOS, and the pattern of frontocentral N3 effects resemble those for the centroparietal N400. Key evidence includes the following. (a) The frontocentral N3 (a.k.a. N390) to visual objects decreases when preceded by semantically-related words and sentences (Federmeier \& Kutas, 2001; Ganis et al., 1996; Kiefer, 2001), which may involve semantic priming. (b) The frontocentral N3 to a picture of a famous face decreases when preceded by vividly imagining that same face (cued by its name) relative to a different picture (Schendan \& Ganis, 2012). This may be a neural correlate of perceptual repetition priming of categorization performance mediated by visual mental imagery of the prime (McDermott \& Roediger, 1994) and/or a mechanism of mental simulation (Schendan \& Ganis, 2012). (c) The frontocentral N3 and RTs decrease for objects categorized following a semantically predictive than nonpredictive, nonlinguistic, visual context specified using a single object (Barrett \& Rugg, 1989, 1990; Holcomb \& McPherson, 1994; McPherson \& Holcomb, 1999), a scene (Ganis \& Kutas, 2003), and a video clip (Sitnikova et al., 2008; Sitnikova et al., 2003). Such scene congruency effects implicate attention processes and the known spatial relations among objects in a scene, and the congruency of the visual knowledge in terms of the spatial position, size, and statistical probability of co-occurrence of the object in the scene (Biederman, 1972, 1981, 1995; Biederman, Blickle, Teitelbaum, \& Klatsky, 1988; Biederman, Glass, \& Stacy, 1973; Biederman, Mezzanotte, \& Rabinowitz, 1982; Biederman, Rabinowitz, Glass, \& Stacy, 1974; Biederman, Teitelbaum, \& Mezzanotte, 1983). (d) The frontocentral N3 shows the earliest target effect (a.k.a. N250; see Section 3.5.1) in ultra-rapid categorization studies, which use a semantic priming variant in which a name (e.g., "animal", "dog") primes the category of a target object (Potter \& Faulconer, 1975) in a scene context. This frontocentral N3 is smaller to scenes with the target than without it from $\sim 150 \mathrm{~ms}$ until $\sim 500 \mathrm{~ms}$ (Bacon-Mace et al., 2005; Delorme et al., 2000; Fabre-Thorpe et al., 2001; J. S. Johnson \& Olshausen, 2003, 2005; R. VanRullen \& S. J. Thorpe, 2001). (e) Crucially for an index of model prediction, impoverishment decreases and delays frontocentral N3 effects (Schendan \& Ganis, 2015; Schendan \& Kutas, 2002, 2003; Schendan \& Maher, 2009). (f) The frontocentral N3 seems to be generated in VLPFC and occipitotemporal cortex, based on intracranial ERPs from 200 to $400 \mathrm{~ms}$ with a mean latency of $250 \mathrm{~ms}$ (Allison et al., 1999; Puce et al., 1999), localization of the frontocentral target $\mathrm{N} 250$ (measured at 190-215 ms) on ultra rapid categorization tasks to occipitotemporal cortex (Delorme et al., 2004; Fize et al., 2005), similar patterns of fMRI effects in VLPFC and LOS for objects in scenes (Gronau et al., 2008; Henderson et al., 2007, 2008; Park et al., 2007), and EEG-guided fMRI evidence that category decision effects with visually impoverished cars and faces reflect interactions between right VLPFC (BA 47/12) and LOS (Philiastides \& Sajda, 2007).

\subsection{PHT Processes Modulate Knowledge}

To explain how PHT modulates knowledge and meaning, MUSI capitalizes on the interactive activation and competition theory of object naming (Humphreys et al., 1999), which suggests how object and word knowledge interact. This theory posits several knowledge systems with recurrent internal connections within each. A 'structural description' system for the perceptual form of object categories is proposed at the entry level to semantics (e.g., dog, car, saw), which interacts reciprocally with a 'semantic representation' system about functional and associative knowledge. This interacts reciprocally with two other systems for 'name representation' and general 'semantic classification' (e.g., animal, vehicle, tool). MUSI hypothesizes the following. (a) To name an 
object, visual object knowledge (e.g., structural descriptions) in the inferotemporal part of the PHT testing system activate during the frontopolar N3. Indeed, left posterior infeiotemporal cortex is implicated in structural descriptions for naming (Humphreys et al., 1999) and shows categorization-specific view effects (Schendan \& Stern, 2008). (b) Semantic representations of objects in the posterior parts (fusiform and LOS) of the PHT prediction system activate during the frontocentral N3. (c) Name representations activate during the centroparietal N400. Notably, these knowledge processes are affected by visual impoverishment during categorization (Schendan \& Ganis, 2015; Schendan \& Kutas, 2003; Schendan \& Lucia, 2012; Schendan et al., submitted). Visual impoverishment affects the frontopolar N3 around $270 \mathrm{~ms}$, the frontocentral N3 component around $310 \mathrm{~ms}$ and the centroparietal N400 around $390 \mathrm{~ms}$ (Schendan et al., submitted), suggesting a temporally overlapping sequence of influence on category decisions from visuospatial knowledge underlying the frontopolar N3, object knowledge underlying the frontocentral N3 and more abstract, multimodal and name-related knowledge underlying the N400. Consistent with this, magnetoencephalographic findings with pictures of real objects indicate that anterior temporal regions implicated as a source of the centroparietal N400 and fusiform regions implicated in model prediction during the frontocentral N3s show more phase locking, which reflects interactive and recurrent processing, with greater semantic integration demands between 200 and $500 \mathrm{~ms}$ (Andrews, Clarke, Pell, \& Hartley, 2010). Altogether, this interactive activity may support automatic simulation to ground cognition, including constructing meaning (Barsalou, 2009; Ganis \& Schendan, 2011).

\subsection{Categorization, Similarity, Novelty and generalization}

Most generally, the MUSI account attempts to explain the human brain's remarkable ability to generalize: To see and decide that novel visual configurations, such as a dog in fog, cloud formations, abstract paintings, or newly created objects (e.g., Segway, cell phone, iPod), are similar to known object structures. Future work should further test the more general hypothesis that PHT networks in state 2 are recruited depending upon the degree of similarity of the visual percept to stored object knowledge, which can vary not only with the degree of image impoverishment but also the statistical regularities of object features and/or their spatial configuration. Evidence for this has been reviewed above. Key additional evidence comes from "pseudo-object" effects, in which pictures of real objects are compared to pictures of pseudoobjects, such as objects with novel features, novel spatial configurations, and real objects combined into a novel figure, and people perform a variety of tasks, such as object or reality decisions, category or memory decisions, or passively view the pictures (e.g., Kroll \& Potter, 1984).. In ERP and fMRI studies, the N3 complex is more negative and the PHT networks are more active for pseudo-objects than real objects (Barbarotto, Laiacona, Macchi, \& Capitani, 2002; Daffner, Mesulam, Scinto, Calvo, et al., 2000; Ganis et al., 2007; Gruber \& Muller, 2005, 2006; Holcomb \& McPherson, 1994; McPherson \& Holcomb, 1999; Michelon, Snyder, Buckner, McAvoy, \& Zacks, 2003; Schendan \& Ganis, 2015; Schendan et al., 1998). Crucially, this is the same pattern as for impoverishment effects, that is, comparisons between more than less impoverished real objects. Together, similarity of impoverishment and pseudo-object effects suggests a more general role for top-down frontoparietal PHT functions. These processes determine that any visual structure that may be ambiguous (e.g., wallet vs. gun held by crime suspect) or novel, and thus visually dissimilar from stored knowledge (e.g., a truck through fog, abstract art, cloud formations, novel artifacts), nonetheless resembles a previously-experienced object.

Thus the MUSI account proposes that PHT processes, such as those in state 2 , serve the more 
general purpose of resolving perceptual discrepancies between perceived input and stored object knowledge, thereby supporting generalization. Such resolution could provide the "teaching" feedback signal to induce new learning about novel visual characteristics, consistent with ERP and fMRI evidence implicating these networks in category learning (Curran et al., 2002; Fazl et al., 2009; Jiang et al., 2007; Little \& Thulborn, 2006; Reber, Gitelman, Parrish, \& Mesulam, 2003; Scott et al., 2006), and evidence that attention processes, which are implicated in PHT (Ganis et al., 2007), can alter visual appearance (Carrasco, Ling, \& Read, 2004). After all, image impoverishment is detrimental to performance because it alters how similar the percept is to stored knowledge (e.g., depth-rotation changes features and global spatial configuration, phase scrambling affects the spatial location of features). Consequently, the visual input is a poor fit to the statistical regularities of the object category. People can decide about the similarity to object knowledge and meaningfulness of novel visual shapes, even geometric shapes and random squiggles (Ganis et al., 2007; Voss \& Paller, 2007; Voss et al., 2010). Most, if not all, images of an object deviate somehow from stored knowledge and memory, and these stored representations are imperfect, even when well-learned (Connor et al., 2007; Ganis et al., 2004; Gold, 2006; M. K. Johnson, 1997; Platt \& Huettel, 2008). Perceptual similarity is a crucial part of a grounded cognition account of categorization (Goldstone, 1994). Most psychological theories of categorization incorporate the idea that an object is automatically determined to be a member of a category based on similarity, and the notion of similarity is constrained by the visual system and processes for integrating multiple sources of similarity information. Perceived shape similarity drives neural responses in monkey inferotemporal and human occipitotemporal cortex and is important for category learning (Cichy et al., 2019; Freedman et al., 2001, 2002, 2003; Jiang et al., 2007; Jozwik et al., 2016; Kriegeskorte, Mur, Ruff, et al., 2008; L. Li et al., 1993; Op de Beeck, Wagemans, et al., 2008; Rainer \& Miller, 2000; Sigala, 2004; Sigala et al., 2002; Sigala \& Logothetis, 2002).

\subsection{Higher-Order Cognition and Task}

The N3, and state 2 processes, in general, reflect processes that use perceptual information, initially computed in state 1 , in the service of higher-order cognitive computations, such as decision-making and achieving task goals, as well as learning, memory encoding and retrieval, especially implicit memory and priming, novelty detection, attentional orienting, automatic mental simulation, and phenomenological consciousness. It is important to highlight that this means that state 2 processes support two general higher-order cognitive abilities: (a) activation of memory, especially knowledge and semantic memory, for higher-order cognition and/ or (b) modulation of higher-order cognition according to task goals (e.g., using cognitive control, attention, and response preparation abilities), but, nonetheless, such higher-order memory computations can happen automatically, regardless of task or decision-making or even without a task during passive viewing. For example, perceptual information can activate memory, especially knowledge and semantic memory, using recurrent and top-down feedback computations to enable higher-order representations (e.g., more global, feature-binding, integrated representations that may require attention as in feature-integration theory (Treisman, 2006)), regardless of task. MUSI theory suggests that recurrent and top-down feedback processes alter the function of a neuron or set of neurons in a cortical area such that they can perform computations that are not possible based on purely bottom-up (feedforward) information processing. Thus the recurrent and top-down feedback processes that are an essential, defining characteristic of state 2 enable a set of neural functions or computations that are not possible in state 1, regardless of task. However, modulation as a function of task or attention is an additional way that functions differ in state 2 compared to 
state 1 . Note, attention and task modulate processing also in state 1 , but the function or the computations achieved as a result of this modulation differs from state 2 . For example, the extreme case of top-down modulation used for strategic mental imagery affects both the N3 in state 2 and the VPP/N170 in state 1, albeit much less in state 1 than 2 (Ganis \& Schendan, 2008; Schendan \& Ganis, 2012). Also, the N3 pseudo-object effect occurs even when the image is task-irrelevant and a non-target stimulus, and the task on targets does not require categorization (e.g., detect red color, Schendan, 1998; Schendan et al., 1998), suggesting higher-order computation of object novelty (i.e., unknown pseudo-object) is a function of state 2, regardless of whether categorization is task relevant. It is also important to emphasize that state 2 involves many higher-order processes, and the N3 is a complex of components such that different components reflect different brain functions (e.g., varying with role in decision-making, memory, and task), and function may vary further with the context or task.. For example, the D220 component reflecting difficulty of two-choice decisions differs between easy and hard perceptual conditions (high vs. low phase coherence) when the task is face-versus-car discrimination, which may be done based on detecting a single feature (e.g., detect eyes), but not when the task is discriminating red-versus-green color (Philiastides et al., 2006). This task-dependence has been taken as evidence for a true top-down influence on decision-making, recruiting task-relevant attention and PHT processes, rather than merely bottomup stimulus processing.

\subsection{State 2 Timing}

One striking feature of mid-latency negativities is that experimental manipulations generally affect amplitude and have little or no effect on latency, and there seem to be lower limits to the onset, consistent with the obligatory nature of neurophysiological states such that state 1 processing needs to reach a point that provides sufficient information for state 2 to begin to operate, and so on. Nonetheless, negativities related to decision making, such as the N2(00), which MUSI hypothesizes is an early component of the N3 complex in state 2 (Folstein \& Van Petten, 2008; Schendan \& Lucia, 2010), can vary in amplitude and latency with response time (Ritter et al., 1979). Further, depending upon the stimuli and task, the onset of N3 effects of category decision success can vary (a) from around 154 to $170 \mathrm{~ms}$ on 2-choice, ultra-rapid categorization tasks, which involve the fastest processing (Thorpe et al., 1996), (b) to between 175 and $218 \mathrm{~ms}$ for pseudo-object effects between intact real categorized objects and pseudo or degraded uncategorized objects on simple tasks (Gruber \& Muller, 2006; Gruber et al., 2006; Holcomb \& McPherson, 1994; McPherson \& Holcomb, 1999; Sehatpour et al., 2006), (c) for impoverishment effects comparing levels of visually degraded drawings between 230 and $255 \mathrm{~ms}$ using a simple object detection task (Schendan \& Ganis, 2015) (d) or between 232 and 364 ms on an open-ended naming task which involves the slowest processing (Doniger et al., 2000; Schendan \& Maher, 2009), (e) with repetition priming, which reduces the onset by over $50 \mathrm{~ms}$ from $316 \mathrm{~ms}$ for new objects down to $252 \mathrm{~ms}$ for repeated objects (Schendan \& Maher, 2009).

\section{STATE M: Details and further evidence}

State M operates after about 300 to $500 \mathrm{~ms}$ and lasts until about $900 \mathrm{~ms}$ or longer to perform internal evaluation and higher-order PHT or verification functions, which can be conscious and effortful. Evaluation functions evaluate internally the accuracy of earlier and ongoing decision and memory processes. Higher-order PHT or verification functions include effortful, strategic, conscious processes for cognitive control, working memory, executive function and mental simulation (i.e., conscious effortful processes involved in mental imagery or mental rotation). These processes have a greater role during more complex cognitive tasks. These brain dynamics 
are reflected in P3b/P3(00)-like late ERPs. These late positive-going ERPs reflect a distinct kind of decision process that uses information from the earlier decision function (e.g., selected/retrieved categories or meanings) to classify stimuli into a task-relevant response type (e.g., Dien, Spencer, \& Donchin, 2004; R. Johnson, Jr., 1984, 1986; Polich, 2007; Sutton \& Ruchkin, 1984).

Categorization success modulates a P3(00)-like ERP, specifically, an occipitoparietal P600 between 500 and $700 \mathrm{~ms}$, which seems related to internally evaluating the success of earlier (e.g., N3) decision processes in state 2 (Schendan \& Kutas, 2002; Schendan \& Maher, 2009) and potentially higher-order semantic processing beyond the N400 . The P600 is more positive for better memory matches, more confident and successful categorization, and canonical than unusual views on categorization (Schendan \& Kutas, 2003), and a semantic P600 has been reported for semantic anomalies in language research (Leckey \& Federmeier, 2019), suggesting a semantic evaluation role (van Herten, Kolk, \& Chwilla, 2005) perhaps related to the name of the object with picture stimuli.

In addition, on categorization or episodic recognition tasks, another late P3-like potential is modulated: the centroparietal late positive complex (LPC). The LPC is an exceptionally large, broadly distributed ERP reflecting large-scale frontoparietal network activity and so likely reflects multiple processes. Later verification of category decisions, complex semantic analysis, and episodic recognition have been associated with a posterior LPC between 500 and $900 \mathrm{~ms}$ (Schendan \& Kutas, 2002; Sitnikova et al., 2008; Voss et al., 2010). The LPC reflects strategic top-down activation of memory, as factors of impoverishment, mental imagery, semantic context, and deception modulate the LPC and do so differently from the earlier N3, thereby dissociating states 2 and M (Ganis, Schendan, \& Keenan, 2013; Schendan \& Ganis, 2012; Schendan \& Lucia, 2009; Schendan \& Maher, 2009). For example, on category decision tasks, the LPC distinguishes between correct and wrong decisions but does not vary with how well the stimulus matches memory, which, by contrast, sensitively modulates the N3 (Schendan \& Kutas, 2002; Schendan \& Maher, 2009).

On episodic memory tasks, numerous studies have established the LPC as a marker of episodic memory retrieval, including recollection. On episodic recognition tasks, the parietal LPC between 500 and $900 \mathrm{~ms}$ is modulated with performance that suggests access to the episodic memory signal in the MTL, being typically more positive for old or familiar than new items, for information remembered better than less well from the study episode, and for high than low confident decisions, especially for old items (e.g., Curran, 2004; Wynn, Daselaar, Kessels, \& Schutter, 2019; Yovel \& Kanwisher, 2004). Notably, even within the MTL, episodic recollection, familiarity and novelty signals can be detected and dissociated (Daselaar, Fleck, \& Cabeza, 2006), suggesting potentially complex interactions depending upon the stimuli, task, and experience. Some theories of recognition suggest that the strength of an episodic memory signal is evaluated for all stimuli, both old and new items. The LPC may reflect, at least in part, this evaluation process itself, not just the episodic memory signal. Consistent with this, the LPC shows a task effect even with unstudied objects that carry no (or minimal) experimental memory signal, being larger on episodic recognition than categorization (Schendan et al., submitted). This LPC modulation is smaller than the characteristic difference between old and new items and may reflect engagement of processes that evaluate the episodic memory signal, including attempts at recollection even when they fail and even with new items. The LPC varies with episodic recollection, as when recalling details of the learning experience during recognition and mental imagery tasks (Rugg \& Curran, 2007; Schendan \& Ganis, 2012). In general, many LPC modulations resemble those in a default mode network that connects strongly with the MTL system for episodic memory and is associated with 
episodic simulation and strategic, conscious mental imagery (Ganis \& Schendan, 2011; Schacter, Addis, \& Buckner, 2008). Such late processes, however, may also support complex semantic analysis beyond the N400 (e.g., Sitnikova, Goff, \& Kuperberg, 2009). Supporting this proposal, while 8-9 year old children show N300 and N400 congruency effects, they have not yet developed the LPC congruency effects seen in adults (Maguire et al., 2013). Thus the LPC reflects internal evaluation and PHT or verification processes that also support strategic, conscious, goal-driven, complex semantic processes, and strategic mental simulation that can contribute to grounding cognition in more abstract and complex ways than earlier automatic mental simulation. Finally, other late ERPs may also operate in state M or constitute a later state, such as a late frontal slowwave after $700 \mathrm{~ms}$ that is observed on categorization and episodic recognition tasks that may reflect response related activity (Schendan \& Ganis, 2015) or retrieval orientation (Schendan et al., submitted) or other late functions.

\subsection{Object Constancy.}

MUSI proposes that state M contributes top-down PHT feedback processes involving strategic contributions from cognitive control, working memory, and selective attention functions in frontoparietal cortex, especially if state 2 PHT processes fail or result in an uncertain decision. According to a hybrid MVPT-verification PHT theory, strategic mental imagery processes, such as mental rotation, contribute to spatial transformation for hypothesis testing after $500 \mathrm{~ms}$. Consistent with this, impoverishment affects processing not only during state 2 but also after 500 ms during state $\mathrm{M}$ (Schendan \& Ganis, 2015; Schendan et al., submitted).

\subsection{Multiple-Network Interactive Dynamics.}

A MUSI account suggests that, to make category and recognition decisions about visual objects, initial bottom-up activation of visual cortical pathways happens in State 1 supporting basic functions like categorical perception. Next, parts of the active task network operate during the N3 complex for higher cognitive functions, especially decision-making, constructing meaning from perceptual information, and automatic simulation for embodied cognition (State 2). Next, State M operates to internally evaluate the earlier decision and memory processes (from earlier states) and strategic mental simulation for embodied cognition during the P600, and any recollection and strategic episodic mental simulation during the LPC and late slow-wave. State M processes involve frontoparietal cortex, the default mode network and the MTL. Notably, these internal evaluations can occur even for new items to serve the task goal of making an appropriate response (e.g., indoor $v s$. outdoor; new vs. old). MUSI proposes that state 2 initially and predominantly reflects active task (or frontoparietal control) and other (e.g., salience or dorsal attention) network activity, whereas state $\mathrm{M}$ includes default mode network activity on explicit memory tasks, such as categorization and especially recognition. This is based on a large body of convergent evidence of similar patterns of effects during state 2 and active task regions versus state $M$ and default mode regions. For example, similar patterns between these states and their respective networks have been seen across parallel ERP (Schendan \& Ganis, 2015; Schendan \& Kutas, 2003; Schendan \& Lucia, 2009, 2010; Schendan et al., submitted) and fMRI studies of categorization, implicit memory, episodic memory, and mental imagery (Ganis et al., 2007; Schendan, submitted; Schendan \& Lucia, 2012; Schendan \& Stern, 2007, 2008).

However, the story is complex and must await future work to clarify. For example, the MUSI account suggests that state 2 is predominantly active task network activity, indexed by N3 complex and N400, and this is consistent with the associated neuroanatomy, but some studies suggest N400 and semantic memory for words at least in part reflects contributions from the default mode network. Semantic memory involved in language comprehension includes modality-specific and 
supramodal (polymodal, multimodal) cortex, including inferior parietal and most of temporal cortex that are parts of the default mode network and convergence zones for multiple perceptual processing pathways (Binder \& Desai, 2011; Binder, Desai, Graves, \& Conant, 2009). Semantic memory and object processing has been proposed, controversially, to extend from occipitotemporal cortex into parahippocampal and even hippocampal regions along a pathway in which semantic memory processes become increasingly abstract and complex and increasingly contribute to episodic memory (e.g., A. C. Lee et al., 2006; C. B. Martin et al., 2016; Quiroga, Kreiman, Koch, \& Fried, 2008). Notably, an N400-P600 complex is often observed during higher cognitive tasks, suggesting a close, perhaps interactive yet dissociable relationship between the underlying neural processes. The MUSI account suggests there may be a transition between highlevel, more abstract semantic memory and episodic memory during the N4-P6 complex.

The MUSI account hypothesizes that the transitions between states are generally dynamic, interactive and complex. Such complexity is illustrated, for example, by the interactive dynamics increasingly understood about the active task networks that predominantly contribute to state 2 and the default mode network regions, including the MTL, that predominantly contribute to state M. The default mode network has a role not only in internal evaluation but also more broadly for externally-directed higher cognition involving a memory-related function (Vatansever, Manktelow, Sahakian, Menon, \& Stamatakis, 2018). The particular combination of network dynamics likely depends upon the stimulus and task processing demands and how these affect allocation of attention. For example, parts of the default mode network may switch attention to the active task network (Corbetta \& Shulman, 2002), and the active task (or frontoparietal control) network is highly interconnected with the default and other networks and mediates internetwork interactions (Spreng et al., 2013).

Consequently, MUSI hypothesizes further that state M reflects interactive dynamics among multiple functional networks, that is, frontoparietal control, salience, and default mode, with the latter having a particularly strong role as a regulator of network dynamics especially when the task or situation recruits episodic memory processes, strategic mental simulation, or internal evaluation of response planning, decisions or memory. For example, divergent thinking processes have a central role in creativity and are associated primarily with default mode network activity, which also interacts dynamically with frontoparietal control (and salience) networks for creative cognition (Zhu et al., 2017). However, given the anti-correlated nature of these networks, each may contribute in state $M$ at different periods of time, and state $M$ likely reflects multiple states, itself (e.g., default mode vs. frontoparietal control state), which is a question for future work.

Further evidence for closely related and interacting yet dissociable processes around the transition between states 2 and 3 focuses on the MTL. This includes evidence that the MTL is independent yet can also be strongly associated with the default mode network during episodic memory tasks (Buckner, Andrews-Hanna, \& Schacter, 2008). Moreover, ERP studies of people with MTL dysfunction indicate that MTL-related activity supports episodic memory function during the LPC and P600, while default mode network activity also contributes to semantic memory function during the P300 and N400. Consider that the MTL supports episodic memory, and circumscribed MTL lesions produce amnesia (i.e., episodic memory problems) and alter the plasticity of the LPC, including the P600 (J. R. Taylor \& Olichney, 2007). In contrast, Alzheimer's dementia affects both the MTL and neocortical association areas of the default mode network, resulting in both episodic and semantic memory problems, and abnormal P300, N400, and P600 (Olichney et al., 2006; Olichney et al., 2013). The MUSI account hypothesizes that the N400 reflects a transformation from modal knowledge and semantic processing during the N3 complex 
in state 2 into supramodal (polymodal, multimodal) higher-level, more abstract semantic processing and word-related knowledge during the N400 in state 2, with the underlying neural generators also contributing to using knowledge and semantic memory to support higher-order semantic analysis during categorization tasks or episodic memory processes in the MTL during episodic memory tasks in state $\mathrm{M}$.

\section{FUTURE DIRECTIONS, CONCLUSIONS \& OVERALL TIME COURSE}

Overall, convergent evidence, especially from ERP studies, supports the MUSI account of the cortical dynamics unfolding over time to support visual object cognition, memory and knowledge (Figures 1-2). The MUSI account proposes, in the first state, initial, bottom-up activation of objectsensitive occipitotemporal cortex occurs between around 95 to $115 \mathrm{~ms}$ to around 150 to $250 \mathrm{~ms}$. State 1 can support several functions: detecting salient shapes against a background (objectsensitivity, between 95-115 and $180 \mathrm{~ms}$, and again after $200 \mathrm{~ms}$ ), categorical perception (between 120 and 170-200 ms), perceptual grouping (between 150 and $250 \mathrm{~ms}$ ), and perceptual implicit learning and memory for these processes However, state 1 cannot support higher cognition or decisions which happen later. Starting between $\sim 150-230 \mathrm{~ms}$ and lasting until around $500 \mathrm{~ms}$ or later, with time course varying with stimulus and task conditions, in state 2 , there is sustained interactive activation of object processing areas, especially in object-sensitive occipitotemporal cortex, that involves top-down, recurrent, and bottom-up processes among these areas and with occipitoparietal and prefrontal cortex. During tasks in which there are little or no expectations (e.g., the response is open-ended or unknown, the stimulus is not pre-cued, no target is specified), Sstate 2 is the first time when lateral prefrontal cortex modulates posterior processing according to the current behavioral context and goal in order to accomplish higher order cognitive functions (e.g., decision-making). This interactive activation provides the earliest, substantial and necessary computations that support decision-making, phenomenological awareness, knowledge retrieval and implicit memory. For example, for a category decision, state 2 supports object model selection: selecting the best match to the percept from among stored object models to make a decision. Further, following initial categorical perception of objects in state 1, interactive, top-down and reflexive feedback, and recurrent processes in state 2 support automatic mental simulation to ground knowledge and meaning (Barsalou, 2009) in modal processing of visual features in occipitotemporal cortex (N3) and multimodal and/ or word-related semantic processes in anterior temporal cortex (N400). Also in state 2, top-down processes of PHT in prefrontal and occipitoparietal cortex can modulate occipitotemporal cortex to enable model selection even with highly impoverished visual images, thereby achieving the visual constancy of object cognition. PHT involves iterative cycles of activity in a brain system for hypothesis prediction and another system for hypothesis testing. PHT processes serve the more general purpose of resolving perceptual discrepancies between perceived input and stored object knowledge and memory: Generalization. The MUSI account hypothesizes that PHT processes in state 2 support object constancy, thereby enabling the remarkable ability to resolve perceptual ambiguity and novelty in the real world -- that is, to see and decide that novel visual shapes resemble known object structures. Such abilities can also enable surrealist or abstract art (e.g., 'Bird in Space' by Brancusi, 1932-1940) to be interpreted as meaningful. State M operates from about 300 to 500 until $900 \mathrm{~ms}$ or longer during P3(00)-like ERPs when internal evaluation and higher-order PHT or verification functions operate, which can be conscious and effortful. These operations can involve a default mode network especially for memory-related functions, which also interacts with other large-scale cortical networks (e.g., frontoparietal, salience). State $M$ supports the second type of mental 
simulation, which is strategic, goal-directed, and conscious and recruited when the task demands internal evaluation of cognition, as in mental imagery and episodic memory tasks. This strategic mental simulation reflects intentional top-down processes involving multiple cortical networks, including lateral prefrontal and posterior parietal networks for attention, cognitive control, and working memory. 


\begin{abstract}
ACKNOWLEDGMENTS
Many thanks especially to Dr. Giorgio Ganis for collaboration and helpful discussions about top-down processing, mental imagery and model verification theory. Many thanks to other collaborators on this research, especially Drs. Stephen M. Kosslyn, Lisa C. Lucia, Stephen M. Maher, Joel Voss, Ken Paller, Marta Kutas, and Chantal E. Stern. Many thanks to Dr. Kara Federmeier for insightful and constructive editorial input on this manuscript. Funding for preparation of this manuscript and development of MUSI theory and evidence by H.E.S. provided by the Research Executive Agency European Union Grants (FP7-PEOPLE-2011-CIG294144 COGNITSIMS and FP7-PEOPLE-2013-ITN-604764 CogNovo), Plymouth University International Research, Networking and Collaboration Grant, American Psychological Association Scientific Conference Grants, National Institute of Mental Health Grant P50 MH071702, National Institute on Aging Grant F32 AG05914, McDonnell-Pew Foundation Fellowships.
\end{abstract}




\section{REFERENCES}

Alcaro, A., Carta, S., \& Panksepp, J. (2017). The Affective Core of the Self: A NeuroArchetypical Perspective on the Foundations of Human (and Animal) Subjectivity. Front Psychol, 8, 1424. doi:10.3389/fpsyg.2017.01424

Allison, T., McCarthy, G., Nobre, A., Puce, A., \& Belger, A. (1994). Human extrastriate visual cortex and the perception of faces, words, numbers, and colors. Cerebral Cortex, 4(5), 544-554.

Allison, T., Puce, A., Spencer, D. D., \& McCarthy, G. (1999). Electrophysiological studies of human face perception. I: Potentials generated in occipitotemporal cortex by face and non-face stimuli. Cerebral Cortex, 9(5), 415-430.

Andrews, T. J., Clarke, A., Pell, P., \& Hartley, T. (2010). Selectivity for low-level features of objects in the human ventral stream. Neuroimage, 49(1), 703-711. doi:10.1016/j.neuroimage.2009.08.046

Aso, T., Hanakawa, T., Matsuo, K., Toma, K., Shibasaki, H., Fukuyama, H., \& Nakai, T. (2007). Subregions of human parietal cortex selectively encoding object orientation. Neurosci Lett, 415(3), 225-230.

Aviezer, H., Landau, A. N., Robertson, L. C., Peterson, M. A., Soroker, N., Sacher, Y., ... Bentin, S. (2007). Implicit integration in a case of integrative visual agnosia. Neuropsychologia, 45(9), 2066-2077.

Bach, P., Gunter, T. C., Knoblich, G., Prinz, W., \& Friederici, A. D. (2009). N400-like negativities in action perception reflect the activation of two components of an action representation. Soc Neurosci, 4(3), 212-232.

Bacon-Mace, N., Mace, M. J., Fabre-Thorpe, M., \& Thorpe, S. J. (2005). The time course of visual processing: backward masking and natural scene categorisation. Vision Res, 45(11), 1459-1469.

Badre, D. (2008). Cognitive control, hierarchy, and the rostro-caudal organization of the frontal lobes. Trends Cogn Sci, 12(5), 193-200.

Badre, D., \& D'Esposito, M. (2007). Functional magnetic resonance imaging evidence for a hierarchical organization of the prefrontal cortex. J Cogn Neurosci, 19(12), 2082-2099.

Badre, D., Hoffman, J., Cooney, J. W., \& D'Esposito, M. (2009). Hierarchical cognitive control deficits following damage to the human frontal lobe. Nat Neurosci, 12(4), 515-522.

Badre, D., \& Wagner, A. D. (2007). Left ventrolateral prefrontal cortex and the cognitive control of memory. Neuropsychologia, 45(13), 2883-2901.

Baird, A. A., Colvin, M. K., Vanhorn, J. D., Inati, S., \& Gazzaniga, M. S. (2005). Functional connectivity: integrating behavioral, diffusion tensor imaging, and functional magnetic resonance imaging data sets. J Cogn Neurosci, 17(4), 687-693.

Baker, C. I., Behrmann, M., \& Olson, C. R. (2002). Impact of learning on representation of parts and wholes in monkey inferotemporal cortex. Nat Neurosci, 5(11), 1210-1216.

Bar, M. (2003). A cortical mechanism for triggering top-down facilitation in visual object recognition. J Cogn Neurosci, 15(4), 600-609.

Bar, M., Kassam, K. S., Ghuman, A. S., Boshyan, J., Schmid, A. M., Dale, A. M., .. . Halgren, E. (2006). Top-down facilitation of visual recognition. Proceedings of the Proceedings of the National Academy of Sciences of the United States of America, 103(2), 449-454.

Barbarotto, R., Laiacona, M., Macchi, V., \& Capitani, E. (2002). Picture reality decision, semantic categories and gender. A new set of pictures, with norms and an experimental study. Neuropsychologia, 40(10), 1637-1653. 
Barrett, S. E., \& Rugg, M. D. (1989). Event-related potentials and the semantic matching of faces. Neuropsychologia, 27(7), 913-922.

Barrett, S. E., \& Rugg, M. D. (1990). Event-related potentials and the semantic matching of pictures. Brain \& Cognition, 14(2), 201-212.

Barsalou, L. W. (2008). Grounded cognition. Annu Rev Psychol, 59, 617-645.

Barsalou, L. W. (2009). Simulation, situated conceptualization, and prediction. Philos Trans $R$ Soc Lond B Biol Sci, 364(1521), 1281-1289.

Behrmann, M., Peterson, M. A., Moscovitch, M., \& Suzuki, S. (2006). Independent representation of parts and the relations between them: evidence from integrative agnosia. J Exp Psychol Hum Percept Perform, 32(5), 1169-1184.

Behrmann, M., \& Williams, P. (2007). Impairments in part-whole representations of objects in two cases of integrative visual agnosia. Cogn Neuropsychol, 24(7), 701-730.

Bentin, S., Allison, T., Puce, A., Perez, E., \& McCarthy, G. (1996). Electrophysiological studies of face perception in humans. Journal of Cognitive Neuroscience, 8(6), 551-565.

Bentin, S., Golland, Y., Flevaris, A., Robertson, L. C., \& Moscovitch, M. (2006). Processing the trees and the forest during initial stages of face perception: electrophysiological evidence. J Cogn Neurosci, 18(8), 1406-1421.

Bentin, S., McCarthy, G., \& Wood, C. C. (1985). Event-related potentials, lexical decision and semantic priming. Electroencephalogr Clin Neurophysiol, 60(4), 343-355.

Biederman, I. (1972). Perceiving real-world scenes. Science, 177(4043), 77-80.

Biederman, I. (1981). Do background depth gradients facilitate object identification? Perception, 10(5), 573-578.

Biederman, I. (1987). Recognition-by-components: A theory of human image understanding. Psychological Review, 94(2), 115-117.

Biederman, I. (1995). Visual object recognition Visual cognition: An invitation to cognitive science, Vol. 2 (2nd ed.). An invitation to cognitive science. (Stephen M. Kosslyn, Daniel N. Osherson, Eds.), (pp. 121-165). Cambridge, MA, US: MIT Press.

Biederman, I., Blickle, T. W., Teitelbaum, R. C., \& Klatsky, G. J. (1988). Object search in nonscene displays. Journal of Experimental Psychology: Learning, Memory, \& Cognition, 14(3), 456-467.

Biederman, I., Glass, A. L., \& Stacy, E. W. (1973). Searching for objects in real-world scenes. J Exp Psychol, 97(1), 22-27.

Biederman, I., Mezzanotte, R. J., \& Rabinowitz, J. C. (1982). Scene perception: Detecting and judging objects undergoing relational violations. Cognitive Psychology, 14(2), 143-177.

Biederman, I., Rabinowitz, J. C., Glass, A. L., \& Stacy, E. W. (1974). On the information extracted from a glance at a scene. J Exp Psychol, 103(3), 597-600.

Biederman, I., Teitelbaum, R. C., \& Mezzanotte, R. J. (1983). Scene perception: A failure to find a benefit from prior expectancy or familiarity. Journal of Experimental Psychology: Learning, Memory, \& Cognition, 9(3), 411-429.

Binder, J. R., \& Desai, R. H. (2011). The neurobiology of semantic memory. Trends Cogn Sci, 15(11), 527-536. doi:10.1016/j.tics.2011.10.001

S1364-6613(11)00214-2 [pii]

Binder, J. R., Desai, R. H., Graves, W. W., \& Conant, L. L. (2009). Where is the semantic system? A critical review and meta-analysis of 120 functional neuroimaging studies. Cerebral Cortex, 19(12), 2767-2796. 
Binney, R. J., Embleton, K. V., Jefferies, E., Parker, G. J., \& Ralph, M. A. (2010). The ventral and inferolateral aspects of the anterior temporal lobe are crucial in semantic memory: evidence from a novel direct comparison of distortion-corrected fMRI, rTMS, and semantic dementia. Cerebral Cortex, 20(11), 2728-2738.

Blanz, V., Tarr, M. J., \& Bulthoff, H. H. (1999). What object attributes determine canonical views? Perception, 28(5), 575-599.

Bookheimer, S. (2002). Functional MRI of language: new approaches to understanding the cortical organization of semantic processing. Annu Rev Neurosci, 25, 151-188.

Borenstein, E., \& Ullman, S. (2008). Combined top-down/bottom-up segmentation. IEEE Trans Pattern Anal Mach Intell, 30(12), 2109-2125.

Bötzel, K., Schulze, S., \& Stodieck, S. R. G. (1995). Scalp topography and analysis of intracranial sources of face-evoked potentials. Experimental Brain Research, 104(1), 135-143.

Boucart, M., Humphreys, G. W., \& Lorenceau, J. (1995). Automatic access to object identity: attention to global information, not to particular physical dimensions, is important. $J$ Exp Psychol Hum Percept Perform, 21(3), 584-601.

Bradski, G., \& Grossberg, S. (1995). Fast-learning VIEWNET architectures for recognizing three-dimensional objects from multiple two-dimensional views. Neural Networks, 8(78), 1053-1080.

Brancusi, C. (1932-1940). Bird in Space (pp. This sculpture, closely related to a marble version completed in 1931, could have been cast as early as 1932 and finished in 1940. Though the shaft of the first Bird in Space was mounted on a discrete conical support, the support of the present example is incorporated as an organically irregular stem, providing an earthbound anchor for the sleek, soaring form. As was customary in Brancusi's work, the brass is smoothed and polished to the point where the materiality of the sculpture is dissolved in its reflective luminosity. Brancusi's spiritual aspirations, his longing for transcendence of the material world and its constraints, are verbalized in his description of Bird in Space as a "project before being enlarged to fill the vault of the sky." $193^{1}$ Lucy Flint).

Brown, R. (2006). What Is a Brain State? Philosophical Psychology, 19(6), 729-742. doi:10.1080/09515080600923271

Brown, R. (2012). The brain and its states. In S. Edelman, T. Fekete, \& N. Zach (Eds.), Being in Time. Dynamical models of phenomenal experience (pp. 211-230): John Benjamins Publishing Company.

Brozinsky, C. J., Yonelinas, A. P., Kroll, N. E., \& Ranganath, C. (2005). Lag-sensitive repetition suppression effects in the anterior parahippocampal gyrus. Hippocampus, 15(5), 557-561.

Buckner, R. L. (2003). Functional-anatomic correlates of control processes in memory. Journal of Neuroscience, 23(10), 3999-4004.

Buckner, R. L., Andrews-Hanna, J. R., \& Schacter, D. L. (2008). The brain's default network: anatomy, function, and relevance to disease. Ann N Y Acad Sci, 1124, 1-38.

Bukach, C. M., Gauthier, I., \& Tarr, M. J. (2006). Beyond faces and modularity: the power of an expertise framework. Trends Cogn Sci, 10(4), 159-166.

Bülthoff, H. H., Edelman, S. Y., \& Tarr, M. J. (1995). How are three-dimensional objects represented in the brain? Cerebral Cortex, 5(3), 247-260.

Bunge, S. A. (2004). How we use rules to select actions: a review of evidence from cognitive neuroscience. Cognitive Affective and Behavioral Neuroscience, 4(4), 564-579. 
Caharel, S., d'Arripe, O., Ramon, M., Jacques, C., \& Rossion, B. (2009). Early adaptation to repeated unfamiliar faces across viewpoint changes in the right hemisphere: evidence from the N170 ERP component. Neuropsychologia, 47(3), 639-643.

Carpenter, P. A., Just, M. A., Keller, T. A., Eddy, W., \& Thulborn, K. (1999). Graded functional activation in the visuospatial system with the amount of task demand. Journal of Cognitive Neuroscience, 11(1), 9-24.

Carrasco, M., Ling, S., \& Read, S. (2004). Attention alters appearance. Nat Neurosci, 7(3), 308313.

Cauquil, A. S., Edmonds, G. E., \& Taylor, M. J. (2000). Is the face-sensitive N170 the only ERP not affected by selective attention? Neuroreport, 11(10), 2167-2171.

Cave, C. B., \& Kosslyn, S. M. (1993). The role of parts and spatial relations in object identification. Perception, 22(2), 229-248.

Chaby, L., Jemel, B., George, N., Renault, B., \& Fiori, N. (2001). An ERP study of famous face incongruity detection in middle age. Brain Cogn, 45(3), 357-377.

Chao, L. L., Haxby, J. V., \& Martin, A. (1999). Attribute-based neural substrates in temporal cortex for perceiving and knowing about objects. Nat Neurosci, 2(10), 913-919.

Chwilla, D. J., Virgillito, D., \& Vissers, C. T. (2011). The relationship of language and emotion: N400 support for an embodied view of language comprehension. J Cogn Neurosci, 23(9), 2400-2414. doi:10.1162/jocn.2010.21578

Cichy, R. M., Kriegeskorte, N., Jozwik, K. M., van den Bosch, J. J. F., \& Charest, I. (2019). The spatiotemporal neural dynamics underlying perceived similarity for real-world objects. Neuroimage, 194, 12-24. doi:10.1016/j.neuroimage.2019.03.031

Cisek, P. (2007). Cortical mechanisms of action selection: the affordance competition hypothesis. Philos Trans R Soc Lond B Biol Sci, 362(1485), 1585-1599.

Cohen, M. S., Kosslyn, S. M., Breiter, H. C., DiGirolamo, G. J., Thompson, W. L., Anderson, A. K., . . Belliveau, J. W. (1996). Changes in cortical activity during mental rotation. A mapping study using functional MRI. Brain, 119(Pt 1)), 89-100.

Connor, C. E., Brincat, S. L., \& Pasupathy, A. (2007). Transformation of shape information in the ventral pathway. Curr Opin Neurobiol, 17(2), 140-147.

Corbetta, M., \& Shulman, G. L. (2002). Control of goal-directed and stimulus-driven attention in the brain. Nat Rev Neurosci, 3(3), 201-215.

Corrigan, N. M., Richards, T., Webb, S. J., Murias, M., Merkle, K., Kleinhans, N. M., . . . Dawson, G. (2009). An investigation of the relationship between fMRI and ERP source localized measurements of brain activity during face processing. Brain Topogr, 22(2), 8396. doi:10.1007/s10548-009-0086-5

Courtney, S. M. (2004). Attention and cognitive control as emergent properties of information representation in working memory. Cognitive Affective and Behavioral Neuroscience, 4(4), 501-516.

Craddock, M., Martinovic, J., \& Muller, M. M. (2015). Early and late effects of objecthood and spatial frequency on event-related potentials and gamma band activity. BMC Neurosci, 16, 6. doi:10.1186/s12868-015-0144-8

Crist, R. E., Wu, C. T., Karp, C., \& Woldorff, M. G. (2007). Face Processing is Gated by Visual Spatial Attention. Front Hum Neurosci, 1, 10.

Curran, T. (2004). Effects of attention and confidence on the hypothesized ERP correlates of recollection and familiarity. Neuropsychologia, 42(8), 1088-1106. 
Curran, T., Tanaka, J. W., \& Weiskopf, D. M. (2002). An electrophysiological comparison of visual categorization and recognition memory. Cognitive Affective and Behavioral Neuroscience, 2(1), 1-18.

Daffner, K. R., Mesulam, M. M., Scinto, L. F., Acar, D., Calvo, V., Faust, R., . . Holcomb, P. (2000). The central role of the prefrontal cortex in directing attention to novel events. Brain, 123 ( Pt 5), 927-939.

Daffner, K. R., Mesulam, M. M., Scinto, L. F., Calvo, V., Faust, R., \& Holcomb, P. J. (2000). An electrophysiological index of stimulus unfamiliarity. Psychophysiology, 37(6), 737-747.

Damasio, A. R. (1989). Time-locked multiregional retroactivation: a systems-level proposal for the neural substrates of recall and recognition. Cognition, 33(1-2), 25-62.

Damasio, A. R. (2010). Self Comes to Mind: Constructing the Conscious Mind. New York, NY: Vintage Books.

Damasio, H., Grabowski, T. J., Tranel, D., Hichwa, R. D., \& Damasio, A. R. (1996). A neural basis for lexical retrieval. Nature, 380(6574), 499-505.

Daselaar, S. M., Fleck, M. S., \& Cabeza, R. (2006). Triple dissociation in the medial temporal lobes: recollection, familiarity, and novelty. J Neurophysiol, 96(4), 1902-1911.

David, O., Harrison, L., \& Friston, K. J. (2005). Modeling event-related responses in the brain. Neuroimage, 25(3), 756-770.

David, O., Kiebel, S. J., Harrison, L. M., Mattout, J., Kilner, J. M., \& Friston, K. J. (2006). Dynamic causal modeling of evoked responses in EEG and MEG. Neuroimage, 30(4), $1255-1272$.

de Fockert, J. W., Rees, G., Frith, C., \& Lavie, N. (2004). Neural correlates of attentional capture in visual search. J Cogn Neurosci, 16(5), 751-759.

de Fockert, J. W., Rees, G., Frith, C. D., \& Lavie, N. (2001). The role of working memory in visual selective attention. Science, 291(5509), 1803-1806.

DeGutis, J., \& D'Esposito, M. (2007). Distinct mechanisms in visual category learning. Cognitive Affective and Behavioral Neuroscience, 7(3), 251-259.

Delorme, A., Richard, G., \& Fabre-Thorpe, M. (2000). Ultra-rapid categorisation of natural scenes does not rely on colour cues: a study in monkeys and humans. Vision Res, 40(16), 2187-2200.

Delorme, A., Rousselet, G. A., Mace, M. J., \& Fabre-Thorpe, M. (2004). Interaction of top-down and bottom-up processing in the fast visual analysis of natural scenes. Cognitive Brain Research, 19, 103-113.

Denys, K., Vanduffel, W., Fize, D., Nelissen, K., Peuskens, H., Van Essen, D., \& Orban, G. A. (2004). The processing of visual shape in the cerebral cortex of human and nonhuman primates: a functional magnetic resonance imaging study. Journal of Neuroscience, 24(10), 2551-2565.

Desimone, R. (1996). Neural mechanisms for visual memory and their role in attention. Proceedings of the Proceedings of the National Academy of Sciences of the United States of America, 93(24), 13494-13499.

Devlin, J. T., Gonnerman, L. M., Andersen, E. S., \& Seidenberg, M. S. (1998). Category-specific semantic deficits in focal and widespread brain damage: a computational account. $J$ Cogn Neurosci, 10(1), 77-94.

Di Lollo, V., Enns, J. T., \& Rensink, R. A. (2000). Competition for consciousness among visual events: the psychophysics of reentrant visual processes. J Exp Psychol Gen, 129(4), 481507. 
Di Russo, F., Martinez, A., Sereno, M. I., Pitzalis, S., \& Hillyard, S. A. (2001). Cortical sources of the early components of the visual evoked potential. Hum Brain Mapp, 15(2), 95-111.

Dien, J., Spencer, K. M., \& Donchin, E. (2004). Parsing the late positive complex: mental chronometry and the ERP components that inhabit the neighborhood of the P300. Psychophysiology, 41(5), 665-678.

Dilkina, K., McClelland, J. L., \& Plaut, D. C. (2008). A single-system account of semantic and lexical deficits in five semantic dementia patients. Cogn Neuropsychol, 25(2), 136-164.

Dobbins, I. G., \& Wagner, A. D. (2005). Domain-general and domain-sensitive prefrontal mechanisms for recollecting events and detecting novelty. Cerebral Cortex, 15(11), 1768-1778.

Dolan, R. J., Fink, G. R., Rolls, E., Booth, M., Holmes, A., Frackowiak, R. S., \& Friston, K. J. (1997). How the brain learns to see objects and faces in an impoverished context. Nature, 389(6651), 596-599.

Doniger, G. M., Foxe, J. J., Murray, M. M., Higgins, B. A., Snodgrass, J. G., Schroeder, C. E., \& Javitt, D. C. (2000). Activation timecourse of ventral visual stream object-recognition areas: High density electrical mapping of perceptual closure processes. Journal of Cognitive Neuroscience, 12(4), 615-621.

Doniger, G. M., Foxe, J. J., Schroeder, C. E., Murray, M. M., Higgins, B. A., \& Javitt, D. C. (2001). Visual perceptual learning in human object recognition areas: a repetition priming study using high-density electrical mapping. Neuroimage, 13(2), 305-313.

Downing, P. E., Chan, A. W., Peelen, M. V., Dodds, C. M., \& Kanwisher, N. (2006). Domain specificity in visual cortex. Cerebral Cortex, 16(10), 1453-1461.

Eger, E., Schyns, P. G., \& Kleinschmidt, A. (2004). Scale invariant adaptation in fusiform faceresponsive regions. Neuroimage, 22(1), 232-242.

Eichenbaum, H. (2000). A cortical-hippocampal system for declarative memory. Nat Rev Neurosci, 1(1), 41-50.

Enns, J. T. (2004). Object substitution and its relation to other forms of visual masking. Vision Res, 44(12), 1321-1331.

Enns, J. T., \& Di Lollo, V. (2000). What's new in visual masking? Trends Cogn Sci, 4(9), 345352.

Enns, J. T., \& Lleras, A. (2008). What's next? New evidence for prediction in human vision. Trends Cogn Sci, 12(9), 327-333.

Enns, J. T., Lleras, A., \& Di Lollo, V. (2006). A Reentrant View of Visual masking, Object substitution, and Response Priming. In H. Ogmen \& B. Breitmeyer (Eds.), The first half second: The microgenesis and temporal dynamics of unconscious and conscious visual processes (pp. 127-147). Cambridge: MIT.

Epstein, R. A., Harris, A., Stanley, D., \& Kanwisher, N. (1999). The parahippocampal place area: recognition, navigation, or encoding? Neuron, 23(1), 115-125.

Epstein, R. A., \& Kanwisher, N. (1998). A cortical representation of the local visual environment. Nature, 392(6676), 598-601.

Fabre-Thorpe, M., Delorme, A., Marlot, C., \& Thorpe, S. (2001). A limit to the speed of processing in ultra-rapid visual categorization of novel natural scenes. J Cogn Neurosci, 13(2), 171-180.

Farah, M. J. (1990). Visual agnosia: Disorders of object recognition and what they tell us about normal vision. Cambridge, Mass.: MIT Press. 
Fazl, A., Grossberg, S., \& Mingolla, E. (2009). View-invariant object category learning, recognition, and search: how spatial and object attention are coordinated using surfacebased attentional shrouds. Cogn Psychol, 58(1), 1-48.

Federmeier, K. D., \& Kutas, M. (2001). Meaning and modality: influences of context, semantic memory organization, and perceptual predictability on picture processing. $J$ Exp Psychol Learn Mem Cogn, 27(1), 202-224.

Felleman, D. J., \& Van Essen, D. C. (1991). Distributed hierarchical processing in the primate cerebral cortex. Cerebral Cortex, 1(1-47).

Fize, D., Fabre-Thorpe, M., Richard, G., Doyon, B., \& Thorpe, S. J. (2005). Rapid categorization of foveal and extrafoveal natural images: associated ERPs and effects of lateralization. Brain Cogn, 59(2), 145-158.

Flevaris, A. V., Robertson, L. C., \& Bentin, S. (2008). Using spatial frequency scales for processing face features and face configuration: an ERP analysis. Brain Res, 1194, 100109.

Fodor, J. A. (1983). The modularity of mind. Cambridge, Massachusetts: MIT Press.

Folstein, J. R., Palmeri, T. J., Van Gulick, A. E., \& Gauthier, I. (2015). Category Learning Stretches Neural Representations in Visual Cortex. Curr Dir Psychol Sci, 24(1), 17-23. doi:10.1177/0963721414550707

Folstein, J. R., \& Van Petten, C. (2004). Multidimensional rule, unidimensional rule, and similarity strategies in categorization: event-related brain potential correlates. $J$ Exp Psychol Learn Mem Cogn, 30(5), 1026-1044.

Folstein, J. R., \& Van Petten, C. (2008). Influence of cognitive control and mismatch on the N2 component of the ERP: a review. Psychophysiology, 45(1), 152-170.

Folstein, J. R., Van Petten, C., \& Rose, S. A. (2008). Novelty and conflict in the categorization of complex stimuli. Psychophysiology, 45(3), 467-479.

Foxe, J. J., \& Simpson, G. V. (2002). Flow of activation from V1 to frontal cortex in humans. A framework for defining "early" visual processing. Exp Brain Res, 142(1), 139-150.

Freedman, D. J., Riesenhuber, M., Poggio, T., \& Miller, E. K. (2001). Categorical representation of visual stimuli in the primate prefrontal cortex. Science, 291(5502), 312-316.

Freedman, D. J., Riesenhuber, M., Poggio, T., \& Miller, E. K. (2002). Visual categorization and the primate prefrontal cortex: neurophysiology and behavior. J Neurophysiol, 88(2), 929941.

Freedman, D. J., Riesenhuber, M., Poggio, T., \& Miller, E. K. (2003). A comparison of primate prefrontal and inferior temporal cortices during visual categorization. Journal of Neuroscience, 23(12), 5235-5246.

Friederici, A. D. (2002). Towards a neural basis of auditory sentence processing. Trends Cogn Sci, 6(2), 78-84.

Friedman-Hill, S. R., Robertson, L. C., Desimone, R., \& Ungerleider, L. G. (2003). Posterior parietal cortex and the filtering of distractors. Proceedings of the Proceedings of the National Academy of Sciences of the United States of America, 100(7), 4263-4268.

Friedman-Hill, S. R., Robertson, L. C., \& Treisman, A. M. (1995). Parietal contributions to visual feature binding: Evidence from a patient with bilateral lesions. Science, 269(5225), 853-855.

Friston, K. (2010). The free-energy principle: a unified brain theory? Nat Rev Neurosci, 11(2), 127-138. 
Friston, K., \& Kiebel, S. (2009). Predictive coding under the free-energy principle. Philos Trans $R$ Soc Lond B Biol Sci, 364(1521), 1211-1221.

Fuster, J. M. (2003). Cortex and Mind: Unifying Cognition. New York: Oxford University Press.

Gabrieli, J. D., Fleischman, D. A., Keane, M. M., Reminger, S. L., \& et al. (1995). Double dissociation between memory systems underlying explicit and implicit memory in the human brain. Psychological Science, 6(2), 76-82.

Gabrieli, J. D., Keane, M. M., Stanger, B. Z., Kjelgaard, M. M., Corkin, S., \& Growdon, J. H. (1994). Dissociations among structural-perceptual, lexical-semantic, and event-fact memory systems in Alzheimer, amnesic, and normal subjects. Cortex, 30(1), 75-103.

Ganis, G., \& Kutas, M. (2003). An electrophysiological study of scene effects on object identification. Brain Res Cogn Brain Res, 16(2), 123-144.

Ganis, G., Kutas, M., \& Sereno, M. I. (1996). The search for common sense: An electrophysiological study of the comprehension of words and pictures in reading. Journal of Cognitive Neuroscience, 8(2), 89-106.

Ganis, G., \& Schendan, H. E. (2008). Visual mental imagery and perception produce opposite adaptation effects on early brain potentials. Neuroimage, 42(4), 1714-1727.

Ganis, G., \& Schendan, H. E. (2011). Mental imagery. Wiley Interdisciplinary Reviews: Cognitive Science, 2(3), 239-252.

Ganis, G., Schendan, H. E., \& Keenan, J. P. (2013). Concealed semantic and episodic autobiographical memory electrified. Frontiers in Psychology, 6(Article 354), 1-21.

Ganis, G., Schendan, H. E., \& Kosslyn, S. M. (2007). Neuroimaging evidence for object model verification theory: Role of prefrontal control in visual object categorization. Neuroimage, 34(1), 384-398.

Ganis, G., Smith, D., \& Schendan, H. E. (2012). The N170, not the P1, indexes the earliest time for categorical perception of faces, regardless of interstimulus variance. Neuroimage, 62(3), 1563-1574.

Ganis, G., Thompson, W. L., \& Kosslyn, S. M. (2004). Brain areas underlying visual mental imagery and visual perception: an fMRI study. Brain Res Cogn Brain Res, 20(2), 226241.

Gauthier, I., Curran, T., Curby, K. M., \& Collins, D. (2003). Perceptual interference supports a non-modular account of face processing. Nat Neurosci, 6(4), 428-432.

Gauthier, I., \& Tarr, M. J. (2016). Visual Object Recognition: Do We (Finally) Know More Now Than We Did? Annu Rev Vis Sci, 2, 377-396. doi:10.1146/annurev-vision-111815114621

Gauthier, I., Tarr, M. J., Anderson, A. W., Skudlarski, P., \& Gore, J. C. (1999). Activation of the middle fusiform 'face area' increases with expertise in recognizing novel objects. Nat Neurosci, 2(6), 568-573.

Gerlach, C. (2007). A review of functional imaging studies on category specificity. J Cogn Neurosci, 19(2), 296-314.

Glenberg, A. M. (1997). What memory is for. Behav Brain Sci, 20(1), 1-19; discussion 19-55.

Gold, J. I. (2006). Multiple roles of experience in decoding the neural representation of sensory stimuli. Novartis Found Symp, 270, 92-101; discussion 101-113.

Gold, J. I., \& Shadlen, M. N. (2007). The neural basis of decision making. Annu Rev Neurosci, $30,535-574$.

Goldrick, M. (2008). Does like attract like? Exploring the relationship between errors and representational structure in connectionist networks. Cogn Neuropsychol, 25(2), 287-313. 
Goldstone, R. L. (1994). The role of similarity in categorization: providing a groundwork. Cognition, 52(2), 125-157.

Goldstone, R. L., \& Barsalou, L. W. (1998). Reuniting perception and conception. Cognition, 65(2-3), 231-262.

Gomez, P., Ratcliff, R., \& Perea, M. (2007). A model of the go/no-go task. J Exp Psychol Gen, 136(3), 389-413.

Gorno-Tempini, M. L., Rankin, K. P., Woolley, J. D., Rosen, H. J., Phengrasamy, L., \& Miller, B. L. (2004). Cognitive and behavioral profile in a case of right anterior temporal lobe neurodegeneration. Cortex, 40(4-5), 631-644.

Gosselin, F., \& Schyns, P. G. (2001). Why do we SLIP to the basic level? Computational constraints and their implementation. Psychol Rev, 108(4), 735-758.

Gotts, S. J. (2016). Incremental learning of perceptual and conceptual representations and the puzzle of neural repetition suppression. Psychon Bull Rev, 23(4), 1055-1071. doi:10.3758/s13423-015-0855-y

Gotts, S. J., Milleville, S. C., Bellgowan, P. S., \& Martin, A. (2011). Broad and Narrow Conceptual Tuning in the Human Frontal Lobes. Cerebral Cortex, 21, 477-491.

Gratton, C., Evans, K. M., \& Federmeier, K. D. (2009). See what I mean? An ERP study of the effect of background knowledge on novel object processing. Mem Cognit, 37(3), 277291.

Graziano, M. S., \& Aflalo, T. N. (2007). Rethinking cortical organization: moving away from discrete areas arranged in hierarchies. Neuroscientist, 13(2), 138-147.

Grezes, J., Armony, J. L., Rowe, J., \& Passingham, R. E. (2003). Activations related to "mirror" and "canonical" neurones in the human brain: an fMRI study. Neuroimage, 18(4), 928937.

Grill-Spector, K., Kourtzi, Z., \& Kanwisher, N. (2001). The lateral occipital complex and its role in object recognition. Vision Res, 41(10-11), 1409-1422.

Grill-Spector, K., Kushnir, T., Edelman, S., Avidan, G., Itzchak, Y., \& Malach, R. (1999). Differential processing of objects under various viewing conditions in the human lateral occipital complex. Neuron, 24, 187-203.

Grill-Spector, K., Kushnir, T., Hendler, T., \& Malach, R. (2000). The dynamics of objectselective activation correlate with recognition performance in humans. Nature Neuroscience, 3(8), 837-843.

Grill-Spector, K., \& Malach, R. (2004). The human visual cortex. Annu Rev Neurosci, 27, 649677.

Gronau, N., Neta, M., \& Bar, M. (2008). Integrated contextual representation for objects' identities and their locations. J Cogn Neurosci, 20(3), 371-388.

Grossberg, S. (1999). The link between brain learning, attention, and consciousness. Conscious Cogn, 8(1), 1-44.

Gruber, T., Malinowski, P., \& Muller, M. M. (2004). Modulation of oscillatory brain activity and evoked potentials in a repetition priming task in the human EEG. Eur J Neurosci, 19(4), 1073-1082.

Gruber, T., \& Muller, M. M. (2005). Oscillatory brain activity dissociates between associative stimulus content in a repetition priming task in the human EEG. Cerebral Cortex, 15(1), 109-116.

Gruber, T., \& Muller, M. M. (2006). Oscillatory brain activity in the human EEG during indirect and direct memory tasks. Brain Res, 1097(1), 194-204. 
Gruber, T., Trujillo-Barreto, N. J., Giabbiconi, C. M., Valdes-Sosa, P. A., \& Muller, M. M. (2006). Brain electrical tomography (BET) analysis of induced gamma band responses during a simple object recognition task. Neuroimage, 29(3), 888-900.

Gu, X., Li, Y., Yang, X., \& Zhu, Y. (2007). Spatial-temporal analysis of face processing using an ERP study of the Thatcher illusion. Conf Proc IEEE Eng Med Biol Soc, 2007, 2496-2499.

Guillaume, F., \& Tiberghien, G. (2001). An event-related potential study of contextual modifications in a face recognition task. Neuroreport, 12(6), 1209-1216.

Guo, C., Lawson, A. L., \& Jiang, Y. (2007). Distinct neural mechanisms for repetition effects of visual objects. Neuroscience, 149(4), 747-759.

Halgren, E., Baudena, P., Clarke, J. M., Heit, G., Liegeois, C., Chauvel, P., \& Musolino, A. (1995). Intracerebral potentials to rare target and distractor auditory and visual stimuli. I. Superior temporal plane and parietal lobe. Electroencephalogr Clin Neurophysiol, 94(3), 191-220.

Halgren, E., Dhond, R. P., Christensen, N., Van Petten, C., Marinkovic, K., Lewine, J. D., \& Dale, A. M. (2002). N400-like magnetoencephalography responses modulated by semantic context, word frequency, and lexical class in sentences. Neuroimage, 17(3), 1101-1116.

Harris, A., \& Nakayama, K. (2007). Rapid face-selective adaptation of an early extrastriate component in MEG. Cerebral Cortex, 17(1), 63-70.

Harris, A., \& Nakayama, K. (2008). Rapid adaptation of the $\mathrm{m} 170$ response: importance of face parts. Cerebral Cortex, 18(2), 467-476.

Harris, I. M., Egan, G. F., Sonkkila, C., Tochon-Danguy, H. J., Paxinos, G., \& Watson, J. D. G. (2000). Selective right parietal lobe activation during mental rotation: A parametric PET study. Brain, 123, 65-73.

Hasson, U., Levy, I., Behrmann, M., Hendler, T., \& Malach, R. (2002). Eccentricity bias as an organizing principle for human high-order object areas. Neuron, 34(3), 479-490.

Haxby, J. V., Gobbini, M. I., Furey, M. L., Ishai, A., Schouten, J. L., \& Pietrini, P. (2001). Distributed and overlapping representations of faces and objects in ventral temporal cortex. Science, 293(5539), 2425-2430.

Heekeren, H. R., Marrett, S., \& Ungerleider, L. G. (2008). The neural systems that mediate human perceptual decision making. Nat Rev Neurosci, 9(6), 467-479.

Heisz, J. J., Watter, S., \& Shedden, J. M. (2006). Progressive N170 habituation to unattended repeated faces. Vision Res, 46(1-2), 47-56.

Henderson, J. M., Larson, C. L., \& Zhu, D. C. (2007). Cortical activation to indoor versus outdoor scenes: an fMRI study. Exp Brain Res, 179(1), 75-84.

Henderson, J. M., Larson, C. L., \& Zhu, D. C. (2008). Full scenes produce more activation than close-up scenes and scene-diagnostic objects in parahippocampal and retrosplenial cortex: an fMRI study. Brain Cogn, 66(1), 40-49.

Henson, R. N. (2003). Neuroimaging studies of priming. Prog Neurobiol, 70(1), 53-81.

Henson, R. N., Goshen-Gottstein, Y., Ganel, T., Otten, L. J., Quayle, A., \& Rugg, M. D. (2003). Electrophysiological and haemodynamic correlates of face perception, recognition and priming. Cerebral Cortex, 13(7), 793-805.

Henson, R. N., \& Rugg, M. D. (2003). Neural response suppression, haemodynamic repetition effects, and behavioural priming. Neuropsychologia, 41(3), 263-270. 
Henson, R. N., Rylands, A., Ross, E., Vuilleumeir, P., \& Rugg, M. D. (2004). The effect of repetition lag on electrophysiological and haemodynamic correlates of visual object priming. Neuroimage, 21(4), 1674-1689.

Hochstein, S., \& Ahissar, M. (2002). View from the top: hierarchies and reverse hierarchies in the visual system. Neuron, 36(5), 791-804.

Holcomb, P. J., Kounios, J., Anderson, J. E., \& West, W. C. (1999). Dual-coding, contextavailability, and concreteness effects in sentence comprehension: an electrophysiological investigation. J Exp Psychol Learn Mem Cogn, 25(3), 721-742.

Holcomb, P. J., \& McPherson, W. B. (1994). Event-related brain potentials reflect semantic priming in an object decision task. Brain and Cognition, 24(2), 259-276.

Horovitz, S. G., Rossion, B., Skudlarski, P., \& Gore, J. C. (2004). Parametric design and correlational analyses help integrating $\mathrm{fMRI}$ and electrophysiological data during face processing. Neuroimage, 22(4), 1587-1595.

Humphreys, G. W., Price, C. J., \& Riddoch, M. J. (1999). From objects to names: a cognitive neuroscience approach. Psychol Res, 62(2-3), 118-130.

Hupe, J. M., James, A. C., Girard, P., Lomber, S. G., Payne, B. R., \& Bullier, J. (2001). Feedback connections act on the early part of the responses in monkey visual cortex. $J$ Neurophysiol, 85(1), 134-145.

Hupe, J. M., James, A. C., Payne, B. R., Lomber, S. G., Girard, P., \& Bullier, J. (1998). Cortical feedback improves discrimination between figure and background by V1, V2 and V3 neurons. Nature, 394(6695), 784-787.

Itier, R. J., \& Taylor, M. J. (2004a). Effects of repetition learning on upright, inverted and contrast-reversed face processing using ERPs. Neuroimage, 21(4), 1518-1532.

Itier, R. J., \& Taylor, M. J. (2004b). N170 or N1? Spatiotemporal differences between object and face processing using ERPs. Cerebral Cortex, 14(2), 132-142.

Itier, R. J., \& Taylor, M. J. (2004c). Source analysis of the N170 to faces and objects. Neuroreport, 15(8), 1261-1265.

Itthipuripat, S., Sprague, T. C., \& Serences, J. T. (2019). Functional MRI and EEG index complementary attentional modulations. Journal of Neuroscience, 39(31), 6162-6179. doi:10.1523/JNEUROSCI.2519-18.2019

Ivry, R. B., \& Robertson, L. C. (1998). The two sides of perception. Cambridge, MA: The Massachusetts Institute of Technology Press.

Jacques, C., Jonas, J., Maillard, L., Colnat-Coulbois, S., Koessler, L., \& Rossion, B. (2019). The inferior occipital gyrus is a major cortical source of the face-evoked N170: Evidence from simultaneous scalp and intracerebral human recordings. Hum Brain Mapp, 40(5), 1403-1418. doi:10.1002/hbm.24455

James, T. W., Humphrey, G. K., Gati, J. S., Menon, R. S., \& Goodale, M. A. (2002). Differential effects of viewpoint on object-driven activation in dorsal and ventral streams. Neuron, 35(4), 793-801.

Jeffreys, D. A. (1996). Evoked potential studies of face and object processing. Visual Cognition, $3(1), 1-38$.

Jemel, B., Schuller, A. M., Cheref-Khan, Y., Goffaux, V., Crommelinck, M., \& Bruyer, R. (2003). Stepwise emergence of the face-sensitive N170 event-related potential component. Neuroreport, 14(16), 2035-2039. 
Jiang, X., Bradley, E., Rini, R. A., Zeffiro, T., Vanmeter, J., \& Riesenhuber, M. (2007). Categorization training results in shape- and category-selective human neural plasticity. Neuron, 53(6), 891-903.

Johnson, J. S., \& Olshausen, B. A. (2003). Timecourse of neural signatures of object recognition. J Vis, 3(7), 499-512.

Johnson, J. S., \& Olshausen, B. A. (2005). The earliest EEG signatures of object recognition in a cued-target task are postsensory. J Vis, 5(4), 299-312.

Johnson, M. K. (1997). Source monitoring and memory distortion. Philos Trans R Soc Lond B Biol Sci, 352(1362), 1733-1745.

Johnson, R., Jr. (1984). P300: a model of the variables controlling its amplitude. Ann N Y Acad Sci, 425, 223-229.

Johnson, R., Jr. (1986). A triarchic model of P300 amplitude. Psychophysiology, 23(4), 367-384.

Joyce, C. A., \& Rossion, B. (2005). The face-sensitive N170 and VPP components manifest the same brain processes: the effect of reference electrode site. Clin Neurophysiol, 116(11), 2613-2631.

Joyce, C. A., Schyns, P. G., Gosselin, F., Cottrell, G. W., \& Rossion, B. (2006). Early selection of diagnostic facial information in the human visual cortex. Vision Res, 46(6-7), 800-813.

Jozwik, K. M., Kriegeskorte, N., \& Mur, M. (2016). Visual features as stepping stones toward semantics: Explaining object similarity in IT and perception with non-negative least squares. Neuropsychologia, 83, 201-226. doi:10.1016/j.neuropsychologia.2015.10.023

Kable, J. W., \& Chatterjee, A. (2006). Specificity of action representations in the lateral occipitotemporal cortex. J Cogn Neurosci, 18(9), 1498-1517.

Kan, I. P., Alexander, M. P., \& Verfaellie, M. (2009). Contribution of prior semantic knowledge to new episodic learning in amnesia. J Cogn Neurosci, 21(5), 938-944.

Kan, I. P., \& Thompson-Schill, S. L. (2004). Effect of name agreement on prefrontal activity during overt and covert picture naming. Cognitive Affective and Behavioral Neuroscience, 4(1), 43-57.

Kanwisher, N., McDermott, J., \& Chun, M. M. (1997). The fusiform face area: A module in human extrastriate cortex specialized for face perception. Journal of Neuroscience, 17(11), 4302-4311.

Kastner, S., \& Pinsk, M. A. (2004). Visual attention as a multilevel selection process. Cognitive Affective and Behavioral Neuroscience, 4(4), 483-500.

Keane, M. M., Gabrieli, J. D., Mapstone, H. C., Johnson, K. A., \& Corkin, S. (1995). Double dissociation of memory capacities after bilateral occipital-lobe or medial temporal-lobe lesions. Brain, 118(Pt 5)), 1129-1148.

Keane, M. M., Gabrieli, J. D. E., Fennema, A. C., Growdon, J. H., \& Corkin, S. (1991). Evidence for a dissociation between perceptual and conceptual priming in Alzheimer's disease. Behavioral Neuroscience, 105(2), 326-342.

Keysers, C., \& Perrett, D. I. (2002). Visual masking and RSVP reveal neural competition. Trends Cogn Sci, 6(3), 120-125.

Kiefer, M. (2001). Perceptual and semantic sources of category-specific effects: event-related potentials during picture and word categorization. Mem Cognit, 29(1), 100-116.

Kim, M. S., \& Robertson, L. C. (2001). Implicit representations of space after bilateral parietal lobe damage. J Cogn Neurosci, 13(8), 1080-1087. 
Kosslyn, S. M., Alpert, N. M., Thompson, W. L., Chabris, C. F., Rauch, S. L., \& Anderson, A. K. (1994). Identifying objects seen from different viewpoints. A PET investigation. Brain, 117(Pt 5), 1055-1071.

Kostopoulos, P., Albanese, M. C., \& Petrides, M. (2007). Ventrolateral prefrontal cortex and tactile memory disambiguation in the human brain. Proceedings of the Proceedings of the National Academy of Sciences of the United States of America, 104(24), 10223-10228.

Kostopoulos, P., \& Petrides, M. (2003). The mid-ventrolateral prefrontal cortex: insights into its role in memory retrieval. Eur J Neurosci, 17(7), 1489-1497.

Kostopoulos, P., \& Petrides, M. (2008a). Left mid-ventrolateral prefrontal cortex: underlying principles of function. Eur J Neurosci, 27(4), 1037-1049.

Kostopoulos, P., \& Petrides, M. (2008b). Waiting to retrieve: possible implications for brain function. Exp Brain Res, 188(1), 91-99.

Kourtzi, Z., \& Kanwisher, N. (2001). Representation of perceived object shape by the human lateral occipital complex. Science, 293(5534), 1506-1509.

Kovacs, G., Zimmer, M., Harza, I., Antal, A., \& Vidnyanszky, Z. (2005). Position-specificity of facial adaptation. Neuroreport, 16(17), 1945-1949.

Kovacs, G., Zimmer, M., Harza, I., \& Vidnyanszky, Z. (2007). Adaptation duration affects the spatial selectivity of facial aftereffects. Vision Res, 47(25), 3141-3149.

Kriegeskorte, N., Mur, M., \& Bandettini, P. (2008). Representational similarity analysis connecting the branches of systems neuroscience. Front Syst Neurosci, 2, 4.

Kriegeskorte, N., Mur, M., Ruff, D. A., Kiani, R., Bodurka, J., Esteky, H., . . . Bandettini, P. A. (2008). Matching categorical object representations in inferior temporal cortex of man and monkey. Neuron, 60(6), 1126-1141.

Kroll, J. F., \& Potter, M. C. (1984). Recognizing words, pictures, and concepts: A comparison of lexical, object, and reality decisions. Journal of Verbal Learning and Verbal Behavior, 23, 39-66.

Kruschke, J. K. (2008). Models of categorization. In R. Sun (Ed.), The Cambridge handbook of computational psychology (pp. 267-301). New York: Cambridge University Press.

Kutas, M., Federmeier, K., Staab, J., \& Kluender, R. (2007). Language. In J. T. Cacioppo, L. Tassinary, \& G. Berntson (Eds.), Handbook of psychophysiology (3rd ed., pp. 555-580). Cambridge: Cambridge University Press.

Kutas, M., \& Federmeier, K. D. (2000). Electrophysiology reveals semantic memory use in language comprehension. Trends Cogn Sci, 4(12), 463-470.

Kutas, M., \& Federmeier, K. D. (2011). Thirty years and counting: finding meaning in the N400 component of the event-related brain potential (ERP). Annu Rev Psychol, 62, 621-647.

Kutas, M., \& Hillyard, S. A. (1980). Reading senseless sentences: Brain potentials reflect semantic incongruity. Science, 207(4427), 203-205.

Lamme, V. A. (2003). Why visual attention and awareness are different. Trends Cogn Sci, 7(1), 12-18.

Lamme, V. A., \& Roelfsema, P. R. (2000). The distinct modes of vision offered by feedforward and recurrent processing. Trends Neurosci, 23(11), 571-579.

Lamme, V. A., Super, H., Landman, R., Roelfsema, P. R., \& Spekreijse, H. (2000). The role of primary visual cortex (V1) in visual awareness. Vision Res, 40(10-12), 1507-1521.

Laszlo, S., \& Federmeier, K. D. (2014). Never Seem to Find the Time: Evaluating the Physiological Time Course of Visual Word Recognition with Regression Analysis of 
Single Item ERPs. Lang Cogn Process, 29(5), 642-661.

doi:10.1080/01690965.2013.866259

Lau, E. F., Phillips, C., \& Poeppel, D. (2008). A cortical network for semantics: (de)constructing the N400. Nat Rev Neurosci, 9(12), 920-933.

Lau, H. C., \& Passingham, R. E. (2007). Unconscious Activation of the Cognitive Control System in the Human Prefrontal Cortex. The Journal of Neuroscience, 27(21), 58055811. doi:10.1523/jneurosci.4335-06.2007

Leckey, M., \& Federmeier, K. D. (2019). The P3b and P600(s): Positive contributions to language comprehension. Psychophysiology, e13351. doi:10.1111/psyp.13351

Ledoux, J., \& Brown, R. (2017). A higher-order theory of emotional consciousness. Proceedings of the National Academy of Sciences, 114(10), E2016-E2025. doi:10.1073/pnas.1619316114

Lee, A. C., Buckley, M. J., Gaffan, D., Emery, T., Hodges, J. R., \& Graham, K. S. (2006). Differentiating the roles of the hippocampus and perirhinal cortex in processes beyond long-term declarative memory: a double dissociation in dementia. Journal of Neuroscience, 26(19), 5198-5203.

Lee, T. S., \& Mumford, D. (2003). Hierarchical Bayesian inference in the visual cortex. J Opt Soc Am A Opt Image Sci Vis, 20(7), 1434-1448.

Lee, T. S., Yang, C. F., Romero, R. D., \& Mumford, D. (2002). Neural activity in early visual cortex reflects behavioral experience and higher-order perceptual saliency. Nat Neurosci, 5(6), 589-597.

Lerner, Y., Hendler, T., Ben-Bashat, D., Harel, M., \& Malach, R. (2001). A hierarchical axis of object processing stages in the human visual cortex. Cerebral Cortex, 11(4), 287-297.

Levy, I., Hasson, U., Avidan, G., Hendler, T., \& Malach, R. (2001). Center-periphery organization of human object areas. Nat Neurosci, 4(5), 533-539.

Li, L., Miller, E. K., \& Desimone, R. (1993). The representation of stimulus familiarity in anterior inferior temporal cortex. Journal of Neurophysiology, 69(6), 1918-1929.

Li, N., \& DiCarlo, J. J. (2008). Unsupervised natural experience rapidly alters invariant object representation in visual cortex. Science, 321(5895), 1502-1507.

Little, D. M., \& Thulborn, K. R. (2006). Prototype-distortion category learning: a two-phase learning process across a distributed network. Brain Cogn, 60(3), 233-243.

Liu, J., Li, J., Rieth, C. A., Huber, D. E., Tian, J., \& Lee, K. (2011). A dynamic causal modeling analysis of the effective connectivities underlying top-down letter processing. Neuropsychologia, 49(5), 1177-1186.

Liu, T., Pestilli, F., \& Carrasco, M. (2005). Transient attention enhances perceptual performance and FMRI response in human visual cortex. Neuron, 45(3), 469-477.

Lowe, D. G. (1985). Perceptual Organisation and Visual Recognition. Boston: Kluwer Academic.

Lowe, D. G. (2000, May). Towards a computational model for object recognition in IT cortex. Paper presented at the First IEEE International Workshop on Biologically Motivated Computer Vision Seoul, Korea.

MacEvoy, S. P., \& Epstein, R. A. (2007). Position selectivity in scene- and object-responsive occipitotemporal regions. J Neurophysiol, 98(4), 2089-2098.

Maguire, M. J., Magnon, G., Ogiela, D. A., Egbert, R., \& Sides, L. (2013). The N300 ERP component reveals developmental changes in object and action identification. Dev Cogn Neurosci, 5, 1-9. doi:10.1016/j.den.2012.11.008 
Maher, S. M. (2011). Cortical Dynamics of Object Categorization and Recognition with Category Exemplars. (Doctor of Philosophy), Tufts University.

Maher, S. M., \& Schendan, H. E. (2008). Neurophysiological investigations of the time course of visual object categorization: Effects of naming, ratings of categorization success, repetition, and exemplar typicality. Paper presented at the Cognitive Neuroscience Society Meeting, San Francisco, CA. .

Maher, S. M., \& Schendan, H. E. (in preparation). Time Course of Exemplar Typicality and Perceptual-Specificity of Implicit Memory during Object Categorization.

Mahon, B. Z., \& Caramazza, A. (2008). A critical look at the embodied cognition hypothesis and a new proposal for grounding conceptual content. J Physiol Paris, 102(1-3), 59-70.

Mahon, B. Z., \& Caramazza, A. (2009). Concepts and categories: a cognitive neuropsychological perspective. Annu Rev Psychol, 60, 27-51.

Malach, R., Reppas, J. B., Benson, R. R., Kwong, K. K., Jiang, H., Kennedy, W. A., . . Tootell, R. B. (1995). Object-related activity revealed by functional magnetic resonance imaging in human occipital cortex. Proceedings of the National Academy of Sciences of the United States of America, 92(18), 8135-8139.

Marinkovic, K., Dhond, R. P., Dale, A. M., Glessner, M., Carr, V., \& Halgren, E. (2003). Spatiotemporal dynamics of modality-specific and supramodal word processing. Neuron, 38(3), 487-497.

Marr, D., \& Nishihara, H. K. (1978). Representation and recognition of the spatial organization of three-dimensional shapes. Proceedings of the Royal Society of London: Series B, 200, 269-294.

Martens, U., Schweinberger, S. R., Kiefer, M., \& Burton, A. M. (2006). Masked and unmasked electrophysiological repetition effects of famous faces. Brain Res, 1109(1), 146-157.

Martin, A. (2007). The representation of object concepts in the brain. Annu Rev Psychol, 58, 2545.

Martin, C. B., Cowell, R. A., Gribble, P. L., Wright, J., \& Kohler, S. (2016). Distributed category-specific recognition-memory signals in human perirhinal cortex. Hippocampus, 26(4), 423-436. doi:10.1002/hipo.22531

McCarthy, G., Puce, A., Belger, A., \& Allison, T. (1999). Electrophysiological studies of human face perception. II: Response properties of face-specific potentials generated in occipitotemporal cortex. Cerebral Cortex, 9(5), 431-444.

McClelland, J. L., \& Rogers, T. T. (2003). The parallel distributed processing approach to semantic cognition. Nat Rev Neurosci, 4(4), 310-322.

McCloskey, M. (2004). Spatial representations and multiple-visual-systems hypotheses: evidence from a developmental deficit in visual location and orientation processing. Cortex, 40(4-5), 677-694.

McDermott, K. B., \& Roediger, H. L., 3rd. (1994). Effects of imagery on perceptual implicit memory tests. J Exp Psychol Learn Mem Cogn, 20(6), 1379-1390.

McMahon, D. B., \& Olson, C. R. (2007). Repetition suppression in monkey inferotemporal cortex: relation to behavioral priming. J Neurophysiol, 97(5), 3532-3543.

McPherson, W. B., \& Holcomb, P. J. (1999). An electrophysiological investigation of semantic priming with pictures of real objects. Psychophysiology, 36(1), 53-65.

Mercure, E., Dick, F., Halit, H., Kaufman, J., \& Johnson, M. H. (2008). Differential lateralization for words and faces: category or psychophysics? J Cogn Neurosci, 20(11), 2070-2087. 
Messinger, A., Squire, L. R., Zola, S. M., \& Albright, T. D. (2005). Neural correlates of knowledge: stable representation of stimulus associations across variations in behavioral performance. Neuron, 48(2), 359-371.

Meyer, K., \& Damasio, A. R. (2009). Convergence and divergence in a neural architecture for recognition and memory. Trends Neurosci, 32(7), 376-382.

Michelon, P., Snyder, A. Z., Buckner, R. L., McAvoy, M., \& Zacks, J. M. (2003). Neural correlates of incongruous visual information. An event-related fMRI study. Neuroimage, 19(4), 1612-1626.

Miki, K., Watanabe, S., Kakigi, R., \& Puce, A. (2004). Magnetoencephalographic study of occipitotemporal activity elicited by viewing mouth movements. Clin Neurophysiol, 115(7), 1559-1574.

Minamimoto, T., Saunders, R. C., \& Richmond, B. J. (2010). Monkeys quickly learn and generalize visual categories without lateral prefrontal cortex. Neuron, 66(4), 501-507.

Montojo, C. A., \& Courtney, S. M. (2008). Differential neural activation for updating rule versus stimulus information in working memory. Neuron, 59(1), 173-182.

Moore, C. J., \& Price, C. J. (1999). Three distinct ventral occipitotemporal regions for reading and object naming. Neuroimage, 10(2), 181-192.

Mouchetant-Rostaing, Y., Giard, M. H., Bentin, S., Aguera, P. E., \& Pernier, J. (2000). Neurophysiological correlates of face gender processing in humans. Eur J Neurosci, 12(1), 303-310.

Mumford, D. (1992). On the computational architecture of the neocortex. II. The role of corticocortical loops. Biol Cybern, 66(3), 241-251.

Murray, E. A., Bussey, T. J., \& Saksida, L. M. (2007). Visual perception and memory: a new view of medial temporal lobe function in primates and rodents. Annu Rev Neurosci, 30, 99-122.

Nobre, A. C., Allison, T., \& McCarthy, G. (1994). Word recognition in the human inferior temporal lobe. Nature, 372(6503), 260-263.

Nobre, A. C., \& McCarthy, G. (1994). Language-related ERPs: Scalp distributions and modulation by word type and semantic priming. Journal of Cognitive Neuroscience, 6(3), 233-255.

Nobre, A. C., \& McCarthy, G. (1995). Language-related field potentials in the anterior-medial temporal lobe: II. Effects of word type and semantic priming. Journal of Neuroscience, 15(2), 1090-1099.

Noppeney, U., Price, C. J., Penny, W. D., \& Friston, K. J. (2006). Two distinct neural mechanisms for category-selective responses. Cerebral Cortex, 16(3), 437-445.

Nunez, P. L., \& Srinivasan, R. (2006). Electric fields of the brain: The neurophysics of EEG (2nd ed.). New York: Oxford University Press.

O'Reilly, R. C., Noelle, D. C., Braver, T. S., \& Cohen, J. D. (2002). Prefrontal cortex and dynamic categorization tasks: representational organization and neuromodulatory control. Cerebral Cortex, 12(3), 246-257.

Olichney, J. M., Iragui, V. J., Salmon, D. P., Riggins, B. R., Morris, S. K., \& Kutas, M. (2006). Absent event-related potential (ERP) word repetition effects in mild Alzheimer's disease. Clin Neurophysiol, 117(6), 1319-1330.

Olichney, J. M., Pak, J., Salmon, D. P., Yang, J. C., Gahagan, T., Nowacki, R., . . IraguiMadoz, V. J. (2013). Abnormal P600 word repetition effect in elderly persons with 
preclinical Alzheimer's disease. Cogn Neurosci, 4(3-4), 143-151.

doi:10.1080/17588928.2013.838945

Olson, C. R. (2001). Object-based vision and attention in primates. Curr Opin Neurobiol, 11(2), 171-179.

Op de Beeck, H. P., Deutsch, J. A., Vanduffel, W., Kanwisher, N. G., \& Dicarlo, J. J. (2007). A Stable Topography of Selectivity for Unfamiliar Shape Classes in Monkey Inferior Temporal Cortex. Cerebral Cortex, 18(7), 1676-1694.

Op de Beeck, H. P., Deutsch, J. A., Vanduffel, W., Kanwisher, N. G., \& DiCarlo, J. J. (2008). A stable topography of selectivity for unfamiliar shape classes in monkey inferior temporal cortex. Cerebral Cortex, 18(7), 1676-1694.

Op de Beeck, H. P., Haushofer, J., \& Kanwisher, N. G. (2008). Interpreting fMRI data: maps, modules and dimensions. Nat Rev Neurosci, 9(2), 123-135.

Op de Beeck, H. P., Wagemans, J., \& Vogels, R. (2008). The representation of perceived shape similarity and its role for category learning in monkeys: a modeling study. Vision Res, 48(4), 598-610.

Oztekin, I., McElree, B., Staresina, B. P., \& Davachi, L. (2009). Working memory retrieval: contributions of the left prefrontal cortex, the left posterior parietal cortex, and the hippocampus. J Cogn Neurosci, 21(3), 581-593.

Paivio, A. (1991). Dual coding theory: Retrospect and current status. Canadian Journal of Psychology, 45(3), 255-287.

Palmer, S. E., Rosch, E., \& Chase, P. (1981). Canonical perspective and the perception of objects. In J. Long \& A. Baddeley (Eds.), Attention and Performance (Vol. IX, pp. 135151). Hillsdale, NJ: Erlbaum.

Panksepp, J., \& Biven, L. (2012). The Archaeology of Mind: Neuroevolutionary Origins of Human Emotions (First ed.). New York, NY: W.W. Norton \& Company, Inc.

Park, S., Intraub, H., Yi, D. J., Widders, D., \& Chun, M. M. (2007). Beyond the edges of a view: boundary extension in human scene-selective visual cortex. Neuron, 54(2), 335-342.

Patterson, K., Nestor, P. J., \& Rogers, T. T. (2007). Where do you know what you know? The representation of semantic knowledge in the human brain. Nat Rev Neurosci, 8(12), 976987.

Peissig, J. J., \& Tarr, M. J. (2007). Visual Object Recognition: Do We Know More Now Than We Did 20 Years Ago? Annu Rev Psychol, 58, 75-96.

Petrides, M. (2005). Lateral prefrontal cortex: architectonic and functional organization. Philos Trans R Soc Lond B Biol Sci, 360(1456), 781-795.

Petrides, M., \& Pandya, D. N. (2001). Comparative cytoarchitectonic analysis of the human and the macaque ventrolateral prefrontal cortex and corticocortical connection patterns in the monkey. Eur J Neurosci, 16(2), 291-310.

Philiastides, M. G., Ratcliff, R., \& Sajda, P. (2006). Neural representation of task difficulty and decision making during perceptual categorization: a timing diagram. Journal of Neuroscience, 26(35), 8965-8975.

Philiastides, M. G., \& Sajda, P. (2006). Temporal characterization of the neural correlates of perceptual decision making in the human brain. Cerebral Cortex, 16(4), 509-518.

Philiastides, M. G., \& Sajda, P. (2007). EEG-informed fMRI reveals spatiotemporal characteristics of perceptual decision making. Journal of Neuroscience, 27(48), 1308213091. 
Pins, D., Meyer, M. E., Foucher, J., Humphreys, G., \& Boucart, M. (2004). Neural correlates of implicit object identification. Neuropsychologia, 42(9), 1247-1259.

Platt, M. L., \& Huettel, S. A. (2008). Risky business: the neuroeconomics of decision making under uncertainty. Nat Neurosci, 11(4), 398-403.

Ploran, E. J., Nelson, S. M., Velanova, K., Donaldson, D. I., Petersen, S. E., \& Wheeler, M. E. (2007). Evidence accumulation and the moment of recognition: dissociating perceptual recognition processes using fMRI. Journal of Neuroscience, 27(44), 11912-11924.

Podzebenko, K., Egan, G. F., \& Watson, J. D. (2002). Widespread dorsal stream activation during a parametric mental rotation task, revealed with functional magnetic resonance imaging. Neuroimage, 15(3), 547-558.

Podzebenko, K., Egan, G. F., \& Watson, J. D. (2005). Real and imaginary rotary motion processing: functional parcellation of the human parietal lobe revealed by fMRI. $J$ Cogn Neurosci, 17(1), 24-36.

Polich, J. (2007). Updating P300: An integrative theory of P3a and P3b. Clinical Neurophysiology, 118, 2128-2148.

Postle, B. R. (2006). Working memory as an emergent property of the mind and brain. Neuroscience, 139(1), 23-38.

Potter, M. C., \& Faulconer, B. A. (1975). Time to understand pictures and words. Nature, 253(5491), 437-438.

Puce, A., Allison, T., \& McCarthy, G. (1999). Electrophysiological studies of human face perception. III: Effects of top-down processing on face-specific potentials. Cerebral Cortex, 9(5), 445-458.

Quiroga, R. Q., Kreiman, G., Koch, C., \& Fried, I. (2008). Sparse but not 'grandmother-cell' coding in the medial temporal lobe. Trends Cogn Sci, 12(3), 87-91.

Rainer, G., \& Miller, E. K. (2000). Effects of visual experience on the representation of objects in the prefrontal cortex. Neuron, 27(1), 179-189.

Raposo, A., Han, S., \& Dobbins, I. G. (2008). Ventrolateral prefrontal cortex and self-initiated semantic elaboration during memory retrieval. Neuropsychologia.

Ratcliff, R. (1978). A theory of memory retrieval. Psychological Review, 85, 59-108.

Ratcliff, R., Philiastides, M. G., \& Sajda, P. (2009). Quality of evidence for perceptual decision making is indexed by trial-to-trial variability of the EEG. Proceedings of the Proceedings of the National Academy of Sciences of the United States of America, 106(16), 65396544.

Reber, P. J., Gitelman, D. R., Parrish, T. B., \& Mesulam, M. M. (2003). Dissociating explicit and implicit category knowledge with fMRI. J Cogn Neurosci, 15(4), 574-583.

Rennie, C. J., Robinson, P. A., \& Wright, J. J. (2002). Unified neurophysical model of EEG spectra and evoked potentials. Biol Cybern, 86(6), 457-471.

Riddoch, M. J., \& Humphreys, G. W. (1987). A case of integrative visual agnosia. Brain, 110 ( Pt 6), 1431-1462.

Riesenhuber, M., \& Poggio, T. (1999). Hierarchical models of object recognition in cortex. Nat Neurosci, 2(11), 1019-1025.

Riesenhuber, M., \& Poggio, T. (2002). Neural mechanisms of object recognition. Curr Opin Neurobiol, 12(2), 162-168.

Riesenhuber, M., \& Poggio, T. (2004). How the visual cortex recognizes objects: the tale of the standard model. In L. M. Chalupa \& J. S. Werner (Eds.), The Visual Neurosciences (Vol. 2, pp. 1640-1653). Cambridge: MIT. 
Ritter, W., Simson, R., Vaughan, H. G., Jr., \& Friedman, D. (1979). A brain event related to the making of a sensory discrimination. Science, 203(4387), 1358-1361.

Robertson, L. C. (2003). Binding, spatial attention and perceptual awareness. Nat Rev Neurosci, $4(2), 93-102$.

Rogers, T. T., \& Patterson, K. (2007). Object categorization: reversals and explanations of the basic-level advantage. J Exp Psychol Gen, 136(3), 451-469.

Rolls, E. T., Tovee, M. J., \& Panzeri, S. (1999). The neurophysiology of backward visual masking: information analysis. J Cogn Neurosci, 11(3), 300-311.

Rosenbaum, R. S., Kohler, S., Schacter, D. L., Moscovitch, M., Westmacott, R., Black, S. E., .. . Tulving, E. (2005). The case of K.C.: contributions of a memory-impaired person to memory theory. Neuropsychologia, 43(7), 989-1021.

Rossell, S. L., Price, C. J., \& Nobre, A. C. (2003). The anatomy and time course of semantic priming investigated by fMRI and ERPs. Neuropsychologia, 41(5), 550-564.

Rossion, B., Campanella, S., Gomez, C. M., Delinte, A., Debatisse, D., Liard, L., . . Guerit, J. M. (1999). Task modulation of brain activity related to familiar and unfamiliar face processing: an ERP study. Clin Neurophysiol, 110(3), 449-462.

Rossion, B., Curran, T., \& Gauthier, I. (2002). A defense of the subordinate-level expertise account for the N170 component. Cognition, 85(2), 189-196.

Rossion, B., \& Jacques, C. (2008). Does physical interstimulus variance account for early electrophysiological face sensitive responses in the human brain? Ten lessons on the N170. Neuroimage, 39(4), 1959-1979.

Rossion, B., Joyce, C. A., Cottrell, G. W., \& Tarr, M. J. (2003). Early lateralization and orientation tuning for face, word, and object processing in the visual cortex. Neuroimage, 20(3), 1609-1624.

Rossion, B., Kung, C. C., \& Tarr, M. J. (2004). Visual expertise with nonface objects leads to competition with the early perceptual processing of faces in the human occipitotemporal cortex. Proceedings of the Proceedings of the National Academy of Sciences of the United States of America, 101(40), 14521-14526.

Rousselet, G. A., Gaspar, C. M., Wieczorek, K. P., \& Pernet, C. R. (2011). Modeling single-trial ERP reveals modulation of bottom-up face visual

processing by top-down task constraints (in some subjects). Frontiers in Psychology, 2(137).

Rousselet, G. A., Husk, J. S., Bennett, P. J., \& Sekuler, A. B. (2005). Spatial scaling factors explain eccentricity effects on face ERPs. J Vis, 5(10), 755-763.

Rousselet, G. A., Husk, J. S., Bennett, P. J., \& Sekuler, A. B. (2008). Time course and robustness of ERP object and face differences. $J$ Vis, 8(12), 3 1-18.

Rousselet, G. A., Mace, M. J., \& Fabre-Thorpe, M. (2004). Animal and human faces in natural scenes: How specific to human faces is the N170 ERP component? J Vis, 4(1), 13-21.

Rousselet, G. A., Thorpe, S. J., \& Fabre-Thorpe, M. (2004). Processing of one, two or four natural scenes in humans: the limits of parallelism. Vision Res, 44(9), 877-894.

Rugg, M. D., \& Curran, T. (2007). Event-related potentials and recognition memory. Trends Cogn Sci, 11(6), 251-257.

Sawamura, H., Georgieva, S., Vogels, R., Vanduffel, W., \& Orban, G. A. (2005). Using functional magnetic resonance imaging to assess adaptation and size invariance of shape processing by humans and monkeys. Journal of Neuroscience, 25(17), 4294-4306. 
Sayala, S., Sala, J. B., \& Courtney, S. M. (2006). Increased neural efficiency with repeated performance of a working memory task is information-type dependent. Cerebral Cortex, 16(5), 609-617.

Schacter, D. L., Addis, D. R., \& Buckner, R. L. (2008). Episodic simulation of future events: concepts, data, and applications. Ann N Y Acad Sci, 1124, 39-60.

Schacter, D. L., \& Tulving, E. (Eds.). (1994). Memory systems 1994. Cambridge, MA, USA: MIT Press.

Schacter, D. L., Wig, G. S., \& Stevens, W. D. (2007). Reductions in cortical activity during priming. Curr Opin Neurobiol, 17, 171-176.

Schendan, H. E. (1998). The timecourse of activation of the neural operations and representations supporting visual object identification and memory. (Doctoral dissertation), University of California, San Diego; 0033, La Jolla.

Schendan, H. E. (submitted). Top-Down Processes from Prefrontal \& Parietal Cortex Support the Perceptual Constancy of Decision-Making \& Implicit Memory.

Schendan, H. E., \& Ganis, G. (2012). Electrophysiological potentials reveal cortical mechanisms for mental imagery, mental simulation, and grounded (embodied) cognition. Front Psychol, 3(Article 329), 1-22. doi:10.3389/fpsyg.2012.00329

Schendan, H. E., \& Ganis, G. (2013). Face-specificity is robust across diverse stimuli and individual people, even when interstimulus variance is zero. Psychophysiology, 50(3), 287-291. doi:10.1111/psyp.12013

Schendan, H. E., \& Ganis, G. (2015). Top-down modulation of visual processing and knowledge after $250 \mathrm{~ms}$ supports object constancy of category decisions. Frontiers in Psychology, 6(Article 1289).

Schendan, H. E., Ganis, G., \& Kutas, M. (1998). Neurophysiological evidence for visual perceptual categorization of words and faces within $150 \mathrm{~ms}$. Psychophysiology, 35(3), 240-251.

Schendan, H. E., \& Kutas, M. (2002). Neurophysiological evidence for two processing times for visual object identification. Neuropsychologia, 40(7), 931-945.

Schendan, H. E., \& Kutas, M. (2003). Time course of processes and representations supporting visual object identification and memory. Journal of Cognitive Neuroscience, 15(1), 111 135. doi:10.1162/089892903321107864

Schendan, H. E., \& Kutas, M. (2007a). Neurophysiological Evidence for the Time Course of Activation of Global Shape, Part, and Local Contour Representations during Visual Object Categorization and Memory. J Cogn Neurosci, 19(5), 734-749.

Schendan, H. E., \& Kutas, M. (2007b). Neurophysiological Evidence for Transfer Appropriate Processing of Memory: Processing versus Feature Similarity. Psychonomic Bulletin \& Review, 14(4), 612-619.

Schendan, H. E., \& Lucia, L. C. (2009). Visual object cognition precedes but also temporally overlaps mental rotation. Brain Research, 1294, 91-105.

Schendan, H. E., \& Lucia, L. C. (2010). Object-Sensitive Activity Reflects Earlier Perceptual and Later Cognitive Processing of Visual Objects between 95 and 500 ms. Brain Research, 1329, 124-141.

Schendan, H. E., \& Lucia, L. C. (2012). Top-Down Processes in Prefrontal \& Parietal Cortex Support the Visual Object Constancy of Category Decisions, Recognition \& Long-Term Implicit Memory after $200 \mathrm{~ms}$. Paper presented at the International Organization of Psychophysiology, Pisa, Italy. 
Schendan, H. E., Lucia, L. C., \& Bridges, D. (submitted). Visual Object Categorization and Episodic Recognition Tasks Recruit Common and Distinct Cortical Processes after 200 ms.

Schendan, H. E., \& Maher, S. M. (2009). Object knowledge during entry-level categorization is activated and modified by implicit memory after $200 \mathrm{~ms}$. Neuroimage, 44(4), 1423-1438.

Schendan, H. E., \& Stern, C. E. (2007). Mental rotation and object categorization share a common network of prefrontal and dorsal and ventral regions of posterior cortex. Neuroimage, 35(3), 1264-1277.

Schendan, H. E., \& Stern, C. E. (2008). Where Vision Meets Memory: Prefrontal-Posterior Networks for Visual Object Constancy during Categorization and Recognition. Cerebral Cortex, 18(7), 1695-1711.

Schoenfeld, M. A., Woldorff, M., Duzel, E., Scheich, H., Heinze, H. J., \& Mangun, G. R. (2003). Form-from-motion: MEG evidence for time course and processing sequence. $J$ Cogn Neurosci, 15(2), 157-172.

Schwarzlose, R. F., Baker, C. I., \& Kanwisher, N. (2005). Separate face and body selectivity on the fusiform gyrus. Journal of Neuroscience, 25(47), 11055-11059.

Schweinberger, S. R., Kaufmann, J. M., Moratti, S., Keil, A., \& Burton, A. M. (2007). Brain responses to repetitions of human and animal faces, inverted faces, and objects: an MEG study. Brain Res, 1184, 226-233.

Schweinberger, S. R., Pickering, E. C., Jentzsch, I., Burton, A. M., \& Kaufmann, J. M. (2002). Event-related brain potential evidence for a response of inferior temporal cortex to familiar face repetitions. Brain Res Cogn Brain Res, 14(3), 398-409.

Schyns, P. G. (1998). Diagnostic recognition: task constraints, object information, and their interactions. Cognition, 67(1-2), 147-179.

Scott, L. S., Tanaka, J. W., Sheinberg, D. L., \& Curran, T. (2006). A reevaluation of the electrophysiological correlates of expert object processing. J Cogn Neurosci, 18(9), 1453-1465.

Sehatpour, P., Molholm, S., Javitt, D. C., \& Foxe, J. J. (2006). Spatiotemporal dynamics of human object recognition processing: An integrated high-density electrical mapping and functional imaging study of "closure" processes. Neuroimage, 29(2), 605-618.

Sehatpour, P., Molholm, S., Schwartz, T. H., Mahoney, J. R., Mehta, A. D., Javitt, D. C., . . Foxe, J. J. (2008). A human intracranial study of long-range oscillatory coherence across a frontal-occipital-hippocampal brain network during visual object processing. Proceedings of the Proceedings of the National Academy of Sciences of the United States of America, 105(11), 4399-4404.

Serre, T., Oliva, A., \& Poggio, T. (2007). A feedforward architecture accounts for rapid categorization. Proceedings of the Proceedings of the National Academy of Sciences of the United States of America, 104(15), 6424-6429.

Serre, T., Wolf, L., Bileschi, S., Riesenhuber, M., \& Poggio, T. (2007). Robust object recognition with cortex-like mechanisms. IEEE Trans Pattern Anal Mach Intell, 29(3), 411-426.

Shepard, R. N. (1987). Toward a universal law of generalization for psychological science. Science, 237(4820), 1317-1323.

Shomstein, S., \& Behrmann, M. (2006). Cortical systems mediating visual attention to both objects and spatial locations. Proceedings of the Proceedings of the National Academy of Sciences of the United States of America, 103(30), 11387-11392. 
Sigala, N. (2004). Visual categorization and the inferior temporal cortex. Behav Brain Res, $149(1), 1-7$.

Sigala, N., Gabbiani, F., \& Logothetis, N. K. (2002). Visual categorization and object representation in monkeys and humans. J Cogn Neurosci, 14(2), 187-198.

Sigala, N., \& Logothetis, N. K. (2002). Visual categorization shapes feature selectivity in the primate temporal cortex. Nature, 415(6869), 318-320.

Simmons, W. K., \& Martin, A. (2009). The anterior temporal lobes and the functional architecture of semantic memory. J Int Neuropsychol Soc, 15(5), 645-649.

Sitnikova, T., Goff, D., \& Kuperberg, G. R. (2009). Neurocognitive abnormalities during comprehension of real-world goal-directed behaviors in schizophrenia. J Abnorm Psychol, 118(2), 256-277.

Sitnikova, T., Holcomb, P. J., Kiyonaga, K. A., \& Kuperberg, G. R. (2008). Two Neurocognitive Mechanisms of Semantic Integration during the Comprehension of Visual Real-world Events. J Cogn Neurosci, 20(11), 2037-2057.

Sitnikova, T., Kuperberg, G., \& Holcomb, P. J. (2003). Semantic integration in videos of realworld events: an electrophysiological investigation. Psychophysiology, 40(1), 160-164.

Sitnikova, T., West, W. C., Kuperberg, G. R., \& Holcomb, P. J. (2006). The neural organization of semantic memory: Electrophysiological activity suggests feature-based segregation. Biol Psychol, 71(3), 326-340.

Smith, M. L., Gosselin, F., \& Schyns, P. G. (2004). Receptive fields for flexible face categorizations. Psychol Sci, 15(11), 753-761.

Snodgrass, J. G., \& Feenan, K. (1990). Priming effects in picture fragment completion: Support for the perceptual closure hypothesis. Journal of Experimental Psychology: General, 119(3), 276-296.

Soldan, A., Mangels, J. A., \& Cooper, L. A. (2006). Evaluating models of object-decision priming: evidence from event-related potential repetition effects. $J$ Exp Psychol Learn Mem Cogn, 32(2), 230-248.

Spreng, R. N., Sepulcre, J., Turner, G. R., Stevens, W. D., \& Schacter, D. L. (2013). Intrinsic architecture underlying the relations among the default, dorsal attention, and frontoparietal control networks of the human brain. J Cogn Neurosci, 25(1), 74-86. doi:10.1162/jocn_a_00281

Squire, L. R., \& Zola-Morgan, S. (1991). The medial temporal lobe memory system. Science, 253(5026), 1380-1386.

Srinivas, K. (1995). Representation of rotated objects in explicit and implicit memory. Journal of Experimental Psychology: Learning, Memory, \& Cognition, 21(4), 1019-1036.

Stahl, J., Wiese, H., \& Schweinberger, S. R. (2010). Learning task affects ERP-correlates of the own-race bias, but not recognition memory performance. Neuropsychologia, 48(7), 2027 2040. doi:S0028-3932(10)00125-9 [pii]

10.1016/j.neuropsychologia.2010.03.024

Stern, C. E., Corkin, S., Gonzalez, R. G., Guimaraes, A. R., Baker, J. R., Jennings, P. J., . . . Rosen, B. R. (1996). The hippocampal formation participates in novel picture encoding: evidence from functional magnetic resonance imaging. Proceedings of the Proceedings of the National Academy of Sciences of the United States of America, 93(16), 8660-8665.

Stern, C. E., Owen, A. M., Look, R. B., Tracey, I., Rosen, B. R., \& Petrides, M. (2000). Activity in ventrolateral and mid-dorsolateral prefrontal cortex during non-spatial visual working 
memory processing: Evidence from functional magnetic resonance imaging. Neuroimage, 11, 392-399.

Sugio, T., Inui, T., Matsuo, K., Matsuzawa, M., Glover, G. H., \& Nakai, T. (1999). The role of the posterior parietal cortex in human object recognition: a functional magnetic resonance imaging study. Neurosci Lett, 276(1), 45-48.

Sutton, S., \& Ruchkin, D. S. (1984). The late positive complex. Advances and new problems. Ann N Y Acad Sci, 425, 1-23.

Swisher, J. D., Halko, M. A., Merabet, L. B., McMains, S. A., \& Somers, D. C. (2007). Visual topography of human intraparietal sulcus. Journal of Neuroscience, 27(20), 5326-5337.

Tanaka, J. W., \& Curran, T. (2001). A neural basis for expert object recognition. Psychol Sci, $12(1), 43-47$.

Tanaka, K. (2003). Columns for complex visual object features in the inferotemporal cortex: clustering of cells with similar but slightly different stimulus selectivities. Cerebral Cortex, 13(1), 90-99.

Tanaka, K., Saito, H., Fukada, Y., \& Moriya, M. (1991). Coding visual images of objects in the inferotemporal cortex of the macaque monkey. Journal of Neurophysiology, 66(1), 170189.

Tarr, M. J., \& Pinker, S. (1989). Mental rotation and orientation-dependence in shape recognition. Cognitive Psychology, 21(2), 233-282.

Tarr, M. J., Williams, P., Hayward, W. G., \& Gauthier, I. (1998). Three-dimensional object recognition is viewpoint dependent. Nature Neuroscience, 1(4), 275-277.

Tartaglia, E. M., Mongillo, G., \& Brunel, N. (2014). On the relationship between persistent delay activity, repetition enhancement and priming. Front Psychol, 5, 1590. doi:10.3389/fpsyg.2014.01590

Taylor, J. R., \& Olichney, J. M. (2007). From amnesia to dementia: ERP studies of memory and language. Clin EEG Neurosci, 38(1), 8-17.

Taylor, K. I., Moss, H. E., Stamatakis, E. A., \& Tyler, L. K. (2006). Binding crossmodal object features in perirhinal cortex. Proceedings of the Proceedings of the National Academy of Sciences of the United States of America, 103(21), 8239-8244.

Thierry, G., Pegna, A. J., Dodds, C., Roberts, M., Basan, S., \& Downing, P. (2006). An eventrelated potential component sensitive to images of the human body. Neuroimage, 32(2), 871-879.

Thompson-Schill, S. L. (2003). Neuroimaging studies of semantic memory: inferring "how" from "where". Neuropsychologia, 41(3), 280-292.

Thorpe, S., Fize, D., \& Marlot, C. (1996). Speed of processing in the human visual system. Nature, 381(6582), 520-522.

Tjan, B. S., Lestou, V., \& Kourtzi, Z. (2006). Uncertainty and invariance in the human visual cortex. J Neurophysiol, 96(3), 1556-1568.

Treisman, A. M. (2006). How the deployment of attention determines what we see. Vis cogn, 14(4-8), 411-443.

Tulving, E. (1972). Episodic and semantic memory. In E. Tulving \& W. Donaldson (Eds.), Organization of memory (pp. 381-403). New York, N.Y.: Academic Press.

Tulving, E., \& Schacter, D. L. (1990). Priming and human memory systems. Science, 247(4940), 301-306. 
Turriziani, P., Carlesimo, G. A., Perri, R., Tomaiuolo, F., \& Caltagirone, C. (2003). Loss of spatial learning in a patient with topographical disorientation in new environments. $J$ Neurol Neurosurg Psychiatry, 74(1), 61-69.

Tyler, L. K., \& Moss, H. E. (2001). Towards a distributed account of conceptual knowledge. Trends Cogn Sci, 5(6), 244-252.

Ullman, S. (1995). Sequence seeking and counter streams: A computational model for bidirectional information flow in the visual cortex. Cerebral Cortex, 5(1), 1-11.

Ullman, S. (1996). High-level vision: Object recognition and visual cognition. Cambridge: MIT.

Ullman, S. (2007). Object recognition and segmentation by a fragment-based hierarchy. Trends Cogn Sci, 11(2), 58-64.

Ullman, S., Sali, E., \& Vidal-Naquet, M. (2001). A Fragment-Based Approach to Object Representation and Classification. In A. Arcelli, L. P. Cordella, \& G. S. d. Baja (Eds.), International Workshop on Visual Form (pp. 85-100). Berlin: Springer.

van Herten, M., Kolk, H. H., \& Chwilla, D. J. (2005). An ERP study of P600 effects elicited by semantic anomalies. Brain Res Cogn Brain Res, 22(2), 241-255. doi:10.1016/j.cogbrainres.2004.09.002

Van Petten, C. (1993). A comparison of lexical and sentence-level context effects in eventrelated potentials. Special Issue: Event-related brain potentials in the study of language. Language \& Cognitive Processes, 8(4), 485-531.

Van Petten, C., Kutas, M., Kluender, R., Mitchiner, M., \& McIsaac, H. K. (1991). Fractionating the word repetition effect with event-related potentials. Journal of Cognitive Neuroscience, 3(2), 131-150.

Van Petten, C., \& Luka, B. J. (2006). Neural localization of semantic context effects in electromagnetic and hemodynamic studies. Brain Lang, 97(3), 279-293.

Vanrie, J., Beatse, E., Wagemans, J., Sunaert, S., \& Van Hecke, P. (2002). Mental rotation versus invariant features in object perception from different viewpoints: an fMRI study. Neuropsychologia, 40(7), 917-930.

VanRullen, R., \& Koch, C. (2003). Visual selective behavior can be triggered by a feed-forward process. Journal of Cognitive Neuroscience, 15, 209-217.

VanRullen, R., \& Thorpe, S. (2001). Ultra-rapid visual categorization of natural and artifactual objects. Perception, 30, 655-668.

VanRullen, R., \& Thorpe, S. J. (2001). The time course of visual processing: from early perception to decision-making. J Cogn Neurosci, 13(4), 454-461.

Vargha-Khadem, F., Gadian, D. G., \& Mishkin, M. (2001). Dissociations in cognitive memory: the syndrome of developmental amnesia. Philos Trans R Soc Lond B Biol Sci, 356(1413), 1435-1440.

Vargha-Khadem, F., Gadian, D. G., Watkins, K. E., Connelly, A., Van Paesschen, W., \& Mishkin, M. (1997). Differential effects of early hippocampal pathology on episodic and semantic memory. Science, 277(5324), 376-380.

Vartanian, O., Beatty, E. L., Smith, I., Blackler, K., Lam, Q., \& Forbes, S. (2018). One-way traffic: The inferior frontal gyrus controls brain activation in the middle temporal gyrus and inferior parietal lobule during divergent thinking. Neuropsychologia. doi:10.1016/j.neuropsychologia.2018.02.024

Vatansever, D., Manktelow, A., Sahakian, B. J., Menon, D. K., \& Stamatakis, E. A. (2018). Default Mode Network Engagement Beyond Self-Referential Internal Mentation. Brain Connect. doi:10.1089/brain.2017.0489 
Verfaellie, M., Cermak, L. S., Blackford, S. P., \& Weiss, S. (1990). Strategic and automatic priming of semantic memory in alcoholic Korsakoff patients. Brain \& Cognition, 13(2), 178-192.

Visser, M., Jefferies, E., \& Lambon Ralph, M. A. (2010). Semantic processing in the anterior temporal lobes: a meta-analysis of the functional neuroimaging literature. $J \operatorname{Cogn}$ Neurosci, 22(6), 1083-1094. doi:10.1162/jocn.2009.21309

Voss, J. L., Federmeier, K. D., \& Paller, K. A. (2012). The potato chip really does look like Elvis! Neural hallmarks of conceptual processing associated with finding novel shapes subjectively meaningful. Cerebral Cortex, 22(10), 2354-2364. doi:10.1093/cercor/bhr315

bhr315 [pii]

Voss, J. L., \& Paller, K. A. (2007). Neural correlates of conceptual implicit memory and their contamination of putative neural correlates of explicit memory. Learn Mem, 14(4), 259267.

Voss, J. L., Schendan, H. E., \& Paller, K. A. (2010). Finding meaning in novel geometric shapes influences electrophysiological correlates of repetition and dissociates perceptual and conceptual priming. Neuroimage, 49(3), 2879-2889. doi:1095-9572 (Electronic)

1053-8119 (Linking)

Warrington, E. K. (1982). Neuropsychological studies of object recognition. Philos Trans R Soc Lond B Biol Sci, 298(1089), 15-33.

Watanabe, S., Kakigi, R., \& Puce, A. (2003). The spatiotemporal dynamics of the face inversion effect: a magneto- and electro-encephalographic study. Neuroscience, 116(3), 879-895.

Wendelken, C., Bunge, S. A., \& Carter, C. S. (2008). Maintaining structured information: an investigation into functions of parietal and lateral prefrontal cortices. Neuropsychologia, 46(2), 665-678.

Wheeler, M. E., Petersen, S. E., Nelson, S. M., Ploran, E. J., \& Velanova, K. (2008). Dissociating early and late error signals in perceptual recognition. $J$ Cogn Neurosci, 20(12), 2211-2225.

Wig, G. S., Grafton, S. T., Demos, K. E., \& Kelley, W. M. (2005). Reductions in neural activity underlie behavioral components of repetition priming. Nat Neurosci, 8(9), 1228-1233.

Willems, R. M., Ozyurek, A., \& Hagoort, P. (2008). Seeing and hearing meaning: ERP and fMRI evidence of word versus picture integration into a sentence context. $J$ Cogn Neurosci, 20(7), 1235-1249.

Wong, A. C., Palmeri, T. J., Rogers, B. P., Gore, J. C., \& Gauthier, I. (2009). Beyond shape: how you learn about objects affects how they are represented in visual cortex. PLoS ONE, 4(12), e8405.

Wynn, S. C., Daselaar, S. M., Kessels, R. P. C., \& Schutter, D. (2019). The electrophysiology of subjectively perceived memory confidence in relation to recollection and familiarity. Brain Cogn, 130, 20-27. doi:10.1016/j.bandc.2018.07.003

$\mathrm{Xu}, \mathrm{Y}$. (2008). Representing connected and disconnected shapes in human inferior intraparietal sulcus. Neuroimage, 40(4), 1849-1856.

$\mathrm{Xu}, \mathrm{Y} .$, \& Chun, M. M. (2006). Dissociable neural mechanisms supporting visual short-term memory for objects. Nature, 440(7080), 91-95.

Xu, Y., \& Chun, M. M. (2007). Visual grouping in human parietal cortex. Proceedings of the Proceedings of the National Academy of Sciences of the United States of America, 104(47), 18766-18771. 
Yang, Y., Tarr, M. J., Kass, R. E., \& Aminoff, E. M. (2019). Exploring spatiotemporal neural dynamics of the human visual cortex. Hum Brain Mapp. doi:10.1002/hbm.24697

Yovel, G., \& Kanwisher, N. (2004). Face perception: domain specific, not process specific. Neuron, 44(5), 889-898.

Zhong, Y. M., \& Rockland, K. S. (2003). Inferior parietal lobule projections to anterior inferotemporal cortex (area TE) in macaque monkey. Cerebral Cortex, 13(5), 527-540.

Zhu, W., Chen, Q., Xia, L., Beaty, R. E., Yang, W., Tian, F., . . Qiu, J. (2017). Common and distinct brain networks underlying verbal and visual creativity. Hum Brain Mapp, 38(4), 2094-2111. doi:10.1002/hbm.23507

\section{FURTHER READING}

Op de Beeck, H. P., Deutsch, J. A., Vanduffel, W., Kanwisher, N. G., \& DiCarlo, J. J. (2008). A stable topography of selectivity for unfamiliar shape classes in monkey inferior temporal cortex. Cerebral Cortex, 18(7), 1676-1694. 